\title{
Error assessment in structural transient dynamics
}

\author{
F. Verdugo ${ }^{1}$, N. Parés ${ }^{1,2}$ and P. Díez ${ }^{1,3 *}$ \\ ${ }^{1}$ Laboratori de Càlcul Numèric (LaCàN), \\ Universitat Politècnica de Catalunya (UPC), \\ Jordi Girona 1-3 E-08034 Barcelona, Spain. \\ ${ }^{2}$ Laboratori de Càlcul Numèric (LaCàN), \\ Escola Universitària d'Enginyeria Tècnica Industrial de Barcelona (EUETIB), \\ Compte d'Urgell, 187, E-08036, Barcelona, Spain. \\ ${ }^{3}$ Centre Internacional de Mètodes Numèrics en Enginyeria (CIMNE), \\ Gran Capitán s/n, E-08034 Barcelona, Spain. \\ Email: \{francesc.verdugo,nuria.pares,pedro.diez\}@upc.edu
}

\begin{abstract}
This paper presents in a unified framework the most representative state-of-the-art techniques on a posteriori error assessment for second order hyperbolic problems, i.e., structural transient dynamics. For the sake of presentation, the error estimates are grouped in four types: recovery-based estimates, the dual weighted residual method, the constitutive relation error method and error estimates for timeline-dependent quantities of interest. All these methodologies give a comprehensive overview on the available error assessment techniques in structural dynamics, both for energy-like and goal-oriented estimates.
\end{abstract}

Keywords: elastodynamics, transient dynamics, goal-oriented error assessment, adjoint problem, quantity of interest, recovery estimates, dual weighted residuals, constitutive relation error, timeline-dependent quantity of interest.

\section{Contents}

1 Introduction 2

2 Problem statement 4

2.1 Strong equations . . . . . . . . . . . . . . . . . . 4

2.2 Weak and discrete formulations . . . . . . . . . . . . . . 4

2.2.1 Newmark-like methods . . . . . . . . . . . . . . 4

2.2.2 Space-time variational formulations . . . . . . . . . . . . 7

*Corresponding author. Tel: +34 93401 7240. Fax: +34 934011825. 
3 Error measures and error representation $\quad 11$

3.1 Errors and error equations . . . . . . . . . . . . . . . 11

3.2 Energy measures . . . . . . . . . . . . . . . . . . . 12

3.3 Quantities of interest and adjoint problem . . . . . . . . . . . 13

3.4 Error representation with adjoint problem . . . . . . . . . . . 17

4 Recovery estimates $\quad 18$

4.1 Space-time error splitting . . . . . . . . . . . . . . . . 19

4.2 Assessing time discretization errors . . . . . . . . . . . . . . 20

4.3 Assessing space discretization errors . . . . . . . . . . . . . . 20

5 Dual weighted residual \& explicit residual estimates 24

5.1 Dual weighted residual method . . . . . . . . . . . . 24

5.2 An $L^{2}$-norm explicit estimate . . . . . . . . . . . . . . 28

6 Constitutive relation error and implicit estimates 31
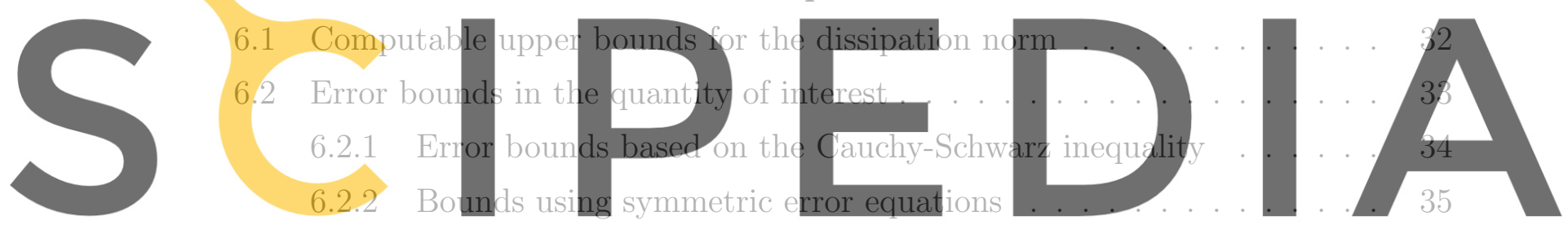

6.2.3 Equivalent alternative approach .............. 36 Register for free at https//www sfipedia.com to download the version without the watermark

6.3.1 The hybrid fluxes method . . . . . . . . . . . . . . 39

6.3.2 The flux-free method . . . . . . . . . . . . . . . . 44

7 Error assessment for timeline-dependent quantities of interest $\quad 45$

7.1 Timeline-dependent quantities of interest . . . . . . . . . . . . 45

7.2 Error representation with family of adjoint problems . . . . . . . . . 46

7.3 Modal-based adjoint approximation . . . . . . . . . . . . . 48

8 Closure $\quad 51$

\section{Introduction}

Discretization errors are intrinsic to any Finite Element (FE) solution. Consequently, the tools assessing and controlling the error or, conversely, the accuracy of the numerical approximation have deserved the attention of the FE community. These tools are especially 
important if sensitive decisions are taken on the basis of the numerical results. Many $a$ posteriori error estimators have been developed with application to different problem types. The application of these techniques to second-order hyperbolic problems (e.g. structural dynamics or elastodynamics) is particularly relevant because, as compared to the standard elliptic problems, the discretization errors are generated and propagated less intuitively or predictively.

The pioneering works on FE error assessment date back to the late 70's and provide estimates of the energy norm of the error in steady-state (elliptic) problems (e.g. linear elasticity or thermal problems), see $[1,2,3]$. Goal-oriented estimates aim at assessing the error of functional outputs of the solution, that is at measuring the error in some Quantity of Interest (QoI). They were introduced much later[4, 5, 6, 7]. In the context of elliptic problems, error estimates are currently pretty well established, both for energy (also denoted as global) and goal-oriented (often referred as local, because the QoI are localized in a particular zone), see $[8,9,10,11,12,13,14]$ as state-of-the-art reviews and books.

The techniques developed for elliptic problems have been extended to other problem types. For instance, quasi-steady-state non-linear problems are addressed in references $[15,16,17,18]$, estimates for advection-diffusion-reaction equation are discussed in [19], similar approaches for the Stokes problem are presented in [20], and extension

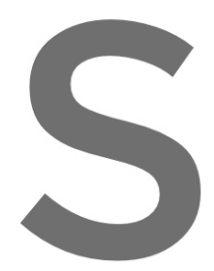
to parabolic time-dependent problems is
type of tools for coupled problems haye b
In the present paper, attention is devoted
structural transient dynamics. In this cont
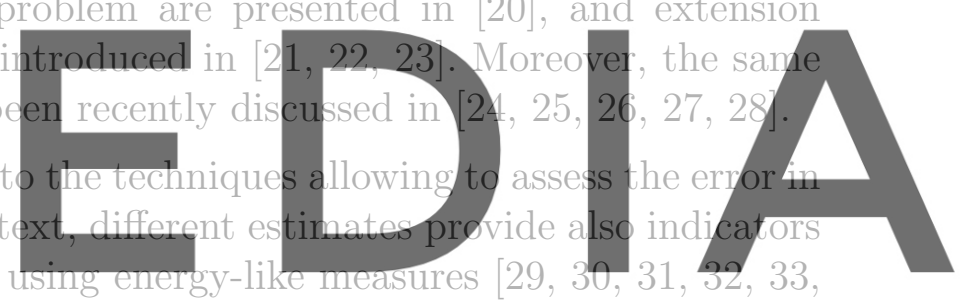

34] or QoI $[35,36,37,38]$. Estimates providing error bounds are also available both for

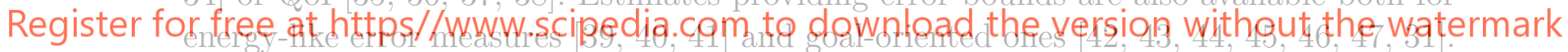

The strategies assessing the error in structural transient dynamics are based on extending the standard estimates designed for steady state linear elasticity. This is still an open problem. The difficulties arise when freezing the time dependence into a series of static problems, in particular in the treatment of the inertia terms.

This review paper aims at presenting the state-of-the-art techniques on a posteriori error assessment for second order hyperbolic problems. Four types of error estimates are analyzed, offering an comprehensive overview: 1) recovery-based estimates, 2) dual weighted residual method, 3) constitutive relation error and 4) error assessment for timelinedependent quantities of interest. The main rationale of each technique is presented following the most representative references. Note that the methodologies presented here are introduced using diverse notations by different authors. Here, the different estimates are described within a unified framework for the sake of an easer reading.

The remainder of the text is organized as follows. Section 2 presents the equations of structural dynamics (both strong and weak versions) and their corresponding approximations. Section 3 introduces the error to be assessed, the error equations and the error representations needed for successive sections. Section 4 is devoted to the recovery-based estimates while section 5 deals with the dual weighted residual method and other ex- 
plicit residual estimates. Section 6 presents the constitutive relation error method and the corresponding error bounds. Finally, section 7 presents an error estimate for special quantities of interest called timeline-dependent quantities of interest. The article is closed with some concluding remarks.

\section{Problem statement}

\subsection{Strong equations}

A visco-elastic body occupies an open bounded domain $\Omega \subset \mathbb{R}^{d}, d \leq 3$, with boundary $\partial \Omega$. The boundary is divided in two disjoint parts, $\Gamma_{\mathrm{N}}$ and $\Gamma_{\mathrm{D}}$ such that $\partial \Omega=\bar{\Gamma}_{\mathrm{N}} \cup \bar{\Gamma}_{\mathrm{D}}$ and the considered time interval is $I:=(0, T]$. Under the assumption of small perturbations, the evolution of displacements $\mathbf{u}(\mathbf{x}, t)$ and stresses $\sigma(\mathrm{x}, t), \mathbf{x} \in \Omega$ and $t \in I$, is described by the yisco-elastodynamic equations,
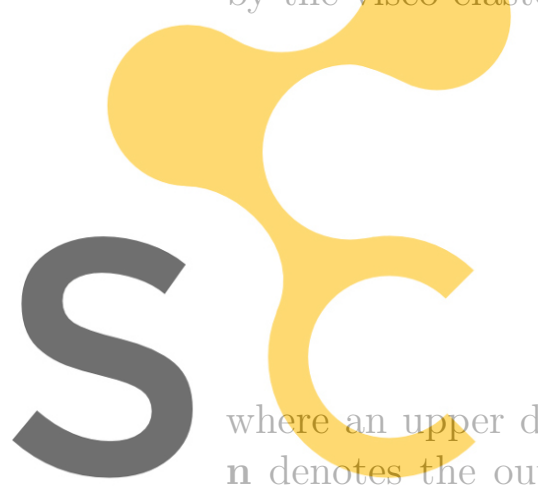

$$
\begin{aligned}
& \rho\left(\ddot{\mathbf{u}}+a_{1} \dot{\mathbf{u}}\right)-\nabla \cdot \sigma=\mathbf{f} \quad \text { in } \Omega \times I, \\
& \mathrm{u}=0 \text { on } \Gamma_{\mathrm{D}} \times I \text {, }
\end{aligned}
$$

$$
\sigma \cdot \mathrm{n}=\mathrm{g} \quad \text { on } \Gamma_{\mathrm{N}} \times I
$$
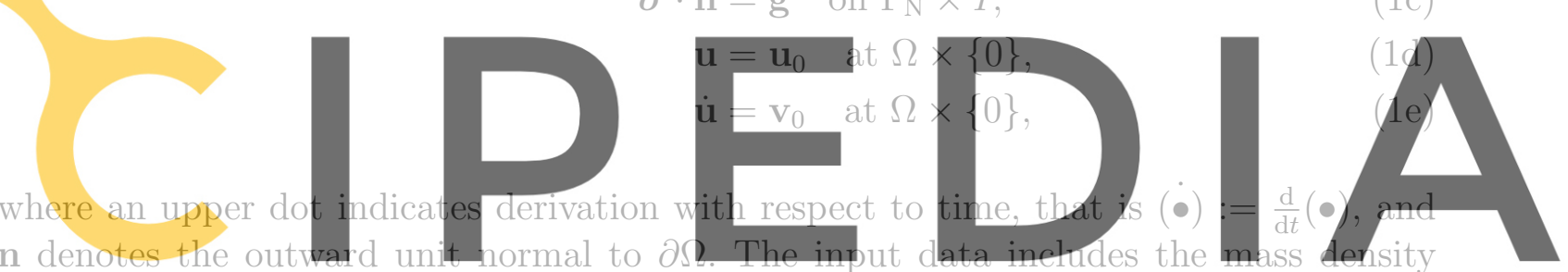

$\rho=\rho(\mathbf{x})>0$, the first Rayleigh coefficient $a_{1} \geq 0$, the body force $\mathbf{f}=\mathbf{f}(\mathbf{x}, t)$ and the

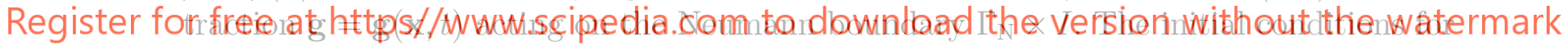

displacements and velocities are $\mathbf{u}_{0}=\mathrm{u}_{0}(\mathrm{x})$ and $\mathrm{v}_{0}=\mathrm{v}_{0}(\mathrm{x})$ respectively. For the sake of simplicity and without any loss of generality, Dirichlet conditions (1b) are taken as homogeneous.

The set of equations (1) is closed with the constitutive law,

$$
\boldsymbol{\sigma}:=\mathcal{C}: \varepsilon\left(\mathbf{u}+a_{2} \dot{\mathbf{u}}\right)
$$

where the parameter $a_{2} \geq 0$ is the second Rayleigh coefficient, the tensor $\mathcal{C}$ is the standard 4th-order elastic Hooke tensor. The strains are given by the kinematic relation corresponding to small perturbations, that is $\varepsilon(\mathbf{w}):=\frac{1}{2}\left(\boldsymbol{\nabla} \mathbf{w}+\nabla^{\mathrm{T}} \mathbf{w}\right)$.

\subsection{Weak and discrete formulations}

\subsubsection{Newmark-like methods}

The definition of the weak form of the problem requires introducing the following functional spaces: the standard Sobolev space associated with static displacement fields

$$
\mathcal{V}_{0}:=\left\{\mathbf{w} \in\left[H^{1}(\Omega)\right]^{d}: \mathbf{w}=\mathbf{0} \text { on } \Gamma_{\mathrm{D}}\right\}
$$


equipped with the usual functional norm which is denoted by $\|\cdot\| \mathcal{V}_{0}$. The Bochner space $L^{2}\left(I ; \mathcal{V}_{0}\right)$ associated with $\mathcal{V}_{0}$ of square-integrable functions from $I$ into $\mathcal{V}_{0}$ is also introduced

$$
L^{2}\left(I ; \mathcal{V}_{0}\right):=\left\{\mathbf{v}: I \rightarrow \mathcal{V}_{0}, \text { such that } \int_{0}^{T}\|\mathbf{v}(t)\|_{\mathcal{V}_{0}}^{2} \mathrm{~d} t<+\infty\right\}
$$

The solution of the problem, $\mathbf{u}(\mathbf{x}, t)$, belongs to the space $\mathcal{W}$ defined as

$$
\mathcal{W}:=\left\{\mathbf{w} \in L^{2}\left(I ; \mathcal{V}_{0}\right) \text { with } \dot{\mathbf{w}} \in L^{2}\left(I ;\left[L^{2}(\Omega)\right]^{d}\right) \text { and } \ddot{\mathbf{w}} \in L^{2}\left(I ; \mathcal{V}_{0}^{\prime}\right)\right\}
$$

where $\mathcal{V}_{0}^{\prime}$ denotes the dual space of $\mathcal{V}_{0}$. Note that in particular this implies that any $\mathbf{w} \in \mathcal{W}$ is such that $\mathbf{w} \in C^{0}\left(\bar{I} ;\left[L^{2}(\Omega)\right]^{d}\right)$ and $\dot{\mathbf{w}} \in C^{0}\left(\bar{I} ; \mathcal{V}_{0}^{\prime}\right)$, see [48]. That is, functions in $\mathcal{W}$ and their time derivatives are continuous in time.

Remark 1. Function $\mathrm{u}$ is a transformation between $\Omega \times I$ and $\mathbb{R}^{d}$, i.e.

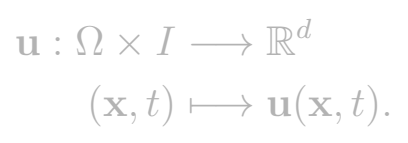

It can also be seen as a transformation between $I$ and $\mathcal{V}_{0}$, i.e.

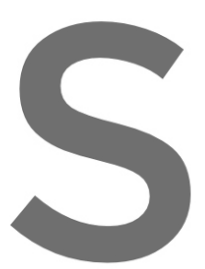

$$
\mathrm{u}: I \longrightarrow \mathcal{V}_{0}
$$

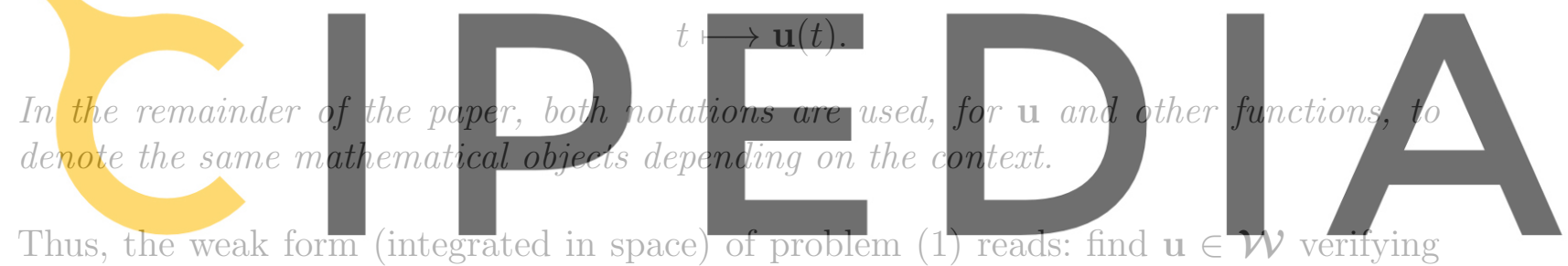

the initial conditions $\mathbf{u}(0)=\mathbf{u}_{0}$.and $\dot{\mathbf{u}}(0)=\mathbf{v}_{0}$ and such that for.all $t \in I$ Register for free at https//www.scipedia.com to download the version without the watermark

$$
m\left(\ddot{\mathbf{u}}(t)+a_{1} \dot{\mathbf{u}}(t), \mathbf{w}\right)+a\left(\mathbf{u}(t)+a_{2} \dot{\mathbf{u}}(t), \mathbf{w}\right)=l(t ; \mathbf{w}) \quad \forall \mathbf{w} \in \mathcal{V}_{0},
$$

where the standard linear and bilinear forms are introduced

$$
\begin{gathered}
a(\mathbf{v}, \mathbf{w}):=\int_{\Omega} \boldsymbol{\varepsilon}(\mathbf{v}): \mathcal{C}: \boldsymbol{\varepsilon}(\mathbf{w}) \mathrm{d} \Omega \quad, \quad m(\mathbf{v}, \mathbf{w}):=\int_{\Omega} \rho \mathbf{v} \cdot \mathbf{w} \mathrm{d} \Omega \\
l(t ; \mathbf{w}):=(\mathbf{f}(t), \mathbf{w})+(\mathbf{g}(t), \mathbf{w})_{\Gamma_{\mathrm{N}}}
\end{gathered}
$$

along with the scalar products

$$
(\mathbf{v}, \mathbf{w}):=\int_{\Omega} \mathbf{v} \cdot \mathbf{w} \mathrm{d} \Omega \quad \text { and } \quad(\mathbf{v}, \mathbf{w})_{\Gamma_{\mathrm{N}}}:=\int_{\Gamma_{\mathrm{N}}} \mathbf{v} \cdot \mathbf{w} \mathrm{d} \Gamma
$$

A mesh of characteristic element size $H$ discretizing the spatial domain $\Omega$ is introduced together with its associated finite element space $\mathcal{V}_{0}^{H} \subset \mathcal{V}_{0}$. The degree of the complete polynomial basis in $\mathcal{V}_{0}^{H}$ is denoted by $p$. This allows introducing the spatially-discrete and time-continuous version of equation (3) (semidiscrete problem), namely: find $\mathbf{u}^{H}(t) \in \mathcal{V}_{0}^{H}$ such that for all $t \in I$

$$
m\left(\ddot{\mathbf{u}}^{H}(t)+a_{1} \dot{\mathbf{u}}^{H}(t), \mathbf{w}\right)+a\left(\mathbf{u}^{H}(t)+a_{2} \dot{\mathbf{u}}^{H}(t), \mathbf{w}\right)=l(t ; \mathbf{w}) \quad \forall \mathbf{w} \in \mathcal{V}_{0}^{H},
$$


with initial conditions (1d) and (1e). In the case $\mathbf{u}_{0}$ and $\mathbf{v}_{0}$ are not in $\mathcal{V}_{0}^{H}$, (1d) and (1e) have to be replaced by $\mathbf{u}^{H}(0)=\boldsymbol{\Pi}^{H}\left(\mathbf{u}_{0}\right)$ and $\dot{\mathbf{u}}^{H}(0)=\boldsymbol{\Pi}^{H}\left(\mathbf{v}_{0}\right)$, being $\boldsymbol{\Pi}^{H}$ the interpolation operator mapping functions from the continuous space $\mathcal{V}_{0}$ into the discrete space $\mathcal{V}_{0}^{H}$.

The Newmark method is a numerical time-marching scheme providing an approximation of the standard system of second order ODEs (4) arising in structural dynamics. A time-grid discretizing the time interval $I$ is introduced, $\mathcal{T}:=\left\{t_{0}, t_{1}, \ldots, t_{N}\right\}$, where $0=t_{0}<t_{1}<\ldots<t_{N}=T$. The time points in $\mathcal{T}$ define the time intervals $I_{n}:=\left(t_{n-1} t_{n}\right]$, $n=1, \ldots, N$. The length of the time interval $I_{n}$ is denoted by $\Delta t_{n}:=t_{n}-t_{n-1}$, for $n=1, \ldots, N$ and the characteristic time step for the time grid is

$$
\Delta t:=\max _{1 \leq n \leq N}\left(\Delta t_{n}\right)
$$

The Newmark solution consists in displacements, velocities and accelerations at each time $t_{n}, \mathbf{u}_{n}^{H, \Delta t} \approx \mathbf{u}^{H}\left(t_{n}\right), \mathbf{v}_{n}^{H, \Delta t} \approx \dot{\mathbf{u}}^{H}\left(t_{n}\right)$ and $\mathbf{a}_{n}^{H, \Delta t} \approx \ddot{\mathbf{u}}^{H}\left(t_{n}\right)$, respectively, for $n=1, \ldots, N$, such that equation (4) is fulfilled at each time $t_{n} \in \mathcal{T}$, that is

$$
m\left(\mathbf{a}_{n}^{H, \Delta t}+a_{1} \mathbf{v}_{n}^{H, \Delta t}, \mathbf{w}\right)+a\left(\mathbf{u}_{n}^{H, \Delta t}+a_{2} \mathbf{v}_{n}^{H, \Delta t}, \mathbf{w}\right)=l_{n}(\mathbf{w}) \quad \forall \mathbf{w} \in \mathcal{V}_{0}^{H} .
$$
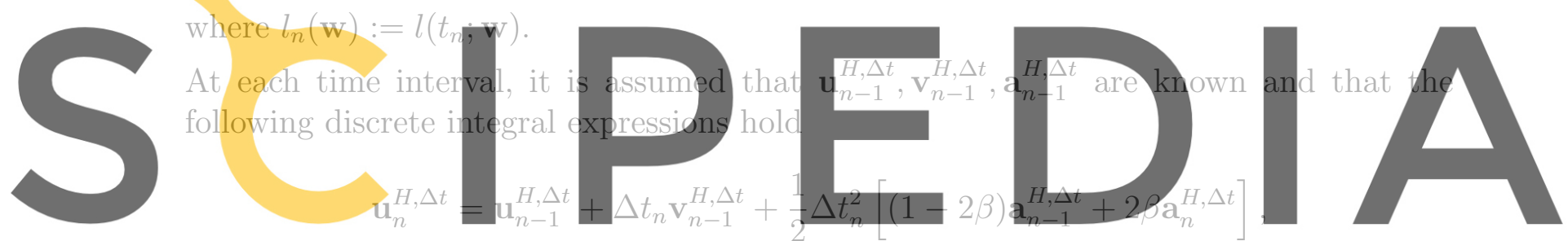

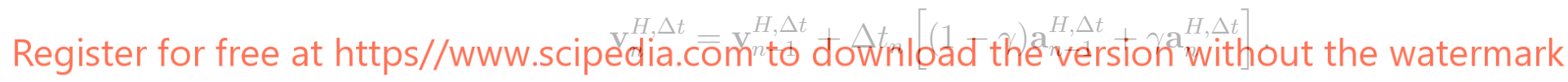

Thus, the only remaining unknown in equation (5) is $\mathrm{a}_{n}^{H, \Delta t}$, which is obtained solving a linear system of algebraic equations. Similarly, at time $t_{0}$, the displacements and velocities are determined by the initial conditions and the acceleration $\mathbf{a}_{0}^{H, \Delta t}$ is computed by considering that

$$
m\left(\mathbf{a}_{0}^{H, \Delta t}+a_{1} \boldsymbol{\Pi}^{H}\left(\mathbf{v}_{0}\right), \mathbf{w}\right)+a\left(\boldsymbol{\Pi}^{H}\left(\mathbf{u}_{0}\right)+a_{2} \boldsymbol{\Pi}^{H}\left(\mathbf{v}_{0}\right), \mathbf{w}\right)=l_{0}(\mathbf{w}) \quad \forall \mathbf{w} \in \mathcal{V}_{0}^{H} .
$$

The scalars $\beta$ and $\gamma$ are the parameters of the Newmark method taking values in $[0,1]$. For $\gamma=1 / 2$ the method is second order accurate and there is no numerical damping, whereas for $\gamma>1 / 2$ numerical damping is introduced. Moreover, the method is conditionally stable for $\beta \geq \gamma / 2 \geq 1 / 4$. See [49] for specific details.

In the framework of using finite difference based time marching schemes, it is quite common that error estimation strategies require obtaining a numerical approximation of problem (1) more regular than the direct numerical solution, with stronger continuity requirements. This post-processed version of the numerical solution is denoted hereafter as $\tilde{\mathbf{u}}$, see for instance section 6 . Note that the Newmark method does not directly provide a numerical approximation $\tilde{\mathbf{u}} \in \mathcal{W}$, since the it is not even defined in the whole time interval $I$ (it is only given at times $t_{n}$ of the time grid). 
The first step in order to recover an smooth numerical approximation is to extend the Newmark approximation into the whole time domain using a simple piecewise linear interpolation:

$$
\begin{aligned}
\mathbf{u}^{H, \Delta t}(\mathbf{x}, t) & :=\sum_{n=0}^{N} \mathbf{u}_{n}^{H, \Delta t}(\mathbf{x}) \theta_{n}(t), \\
\mathbf{v}^{H, \Delta t}(\mathbf{x}, t) & :=\sum_{n=0}^{N} \mathbf{v}_{n}^{H, \Delta t}(\mathbf{x}) \theta_{n}(t), \\
\mathbf{a}^{H, \Delta t}(\mathbf{x}, t) & :=\sum_{n=0}^{N} \mathbf{a}_{n}^{H, \Delta t}(\mathbf{x}) \theta_{n}(t),
\end{aligned}
$$

where the functions $\theta_{n}(t)$, for $n=0, \ldots, N$, are the one-dimensional piecewise linear shape functions related with the time partition $\mathcal{T}$. Note that, however, one cannot take $\tilde{\mathbf{u}}=\mathbf{u}^{H, \Delta t}(\mathbf{x}, t)$ since this approximation does not meet the regularity requirements of the functional space $\mathcal{W}, \mathbf{u}^{H, \Delta t}(\mathbf{x}, t) \notin \mathcal{W}$, because its time derivative is not continuous. Following [9], an admissible approximation $\tilde{\mathbf{u}} \in \mathcal{W}$ is easily recovered from the Newmark solution using the information provided by the numerical accelerations, namely
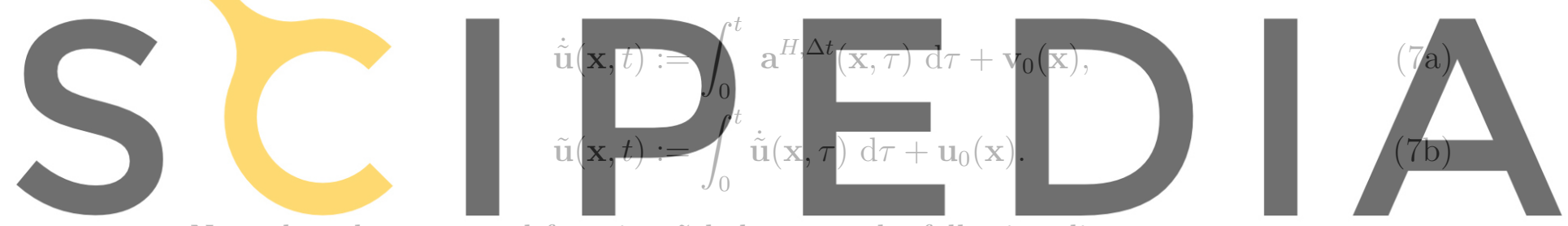

Note that the recovered function $\tilde{u}$ belongs to the following discrete space

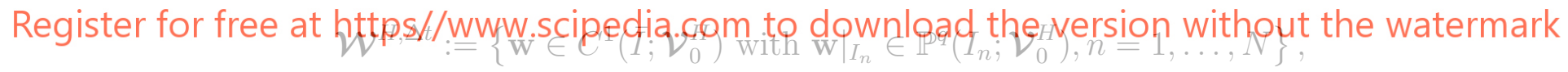

where $\mathbb{P}^{q}(\cdot)$ represent the space of polynomials of order $q$ in time (where for the particular expression (7b), the polynomial order is $q=3$ ). Note that by construction the approximation $\tilde{\mathbf{u}}$ exactly verifies the initial conditions and that the admissible acceleration coincides with the Newmark solution, $\ddot{\tilde{\mathbf{u}}}=\mathbf{a}^{H, \Delta t}$. Note that the displacements $\mathbf{u}^{H, \Delta t}$ and $\tilde{\mathbf{u}}$ do not coincide but they converge to the same function as $\Delta t$ tends to zero.

\subsubsection{Space-time variational formulations}

The main objective of a posteriori error estimation techniques is to evaluate the error in some specific scalar measure (energy-type norms or quantities of interest). The error is related with the non-verification of the equation to be solved, that is with the residual. In order to properly define the residual associated with some numerical approximation, a space-time variational form of the problem is required. Note that the variational format is employed to derive the error estimate, not necessarily to solve the problem. For instance, the Newmark method is not using any time variational form. However, there are some a posteriori error estimation techniques using the full variational formulation both for the problem approximation and for the error assessment strategy, see [35, 50, 29, 30]. 
Among the possible space-time variational formulations available for transient elastodynamics, four options are considered in the remainder of the paper. They correspond to the choices made by the authors that designed the error estimation strategies presented here. The first option is a Single Field (SF) Galerkin method based on the approach introduced by Hughes and Hulbert $[51,52]$. The other three options follow a Double Field (DF) formulation. The second option, based on [35], is a time-Continuous Galerkin approach using the mass product $m(\cdot, \cdot)$ to enforce the displacement-velocity consistency, and it will be referred as CGM. The third option, denoted by CGA, very similar to the previous one, differs in the fact that displacement-velocity consistency is enforced using the bilinear form $a(\cdot, \cdot)$, see [50]. The fourth case is a double field discontinuous Galerkin method introduced by Johnson [53] and it is denoted by DG.

The SF approach uses the following weak form of problem (1) (the SF reference is omitted in the notation): find $u \in \mathcal{W}$ such that

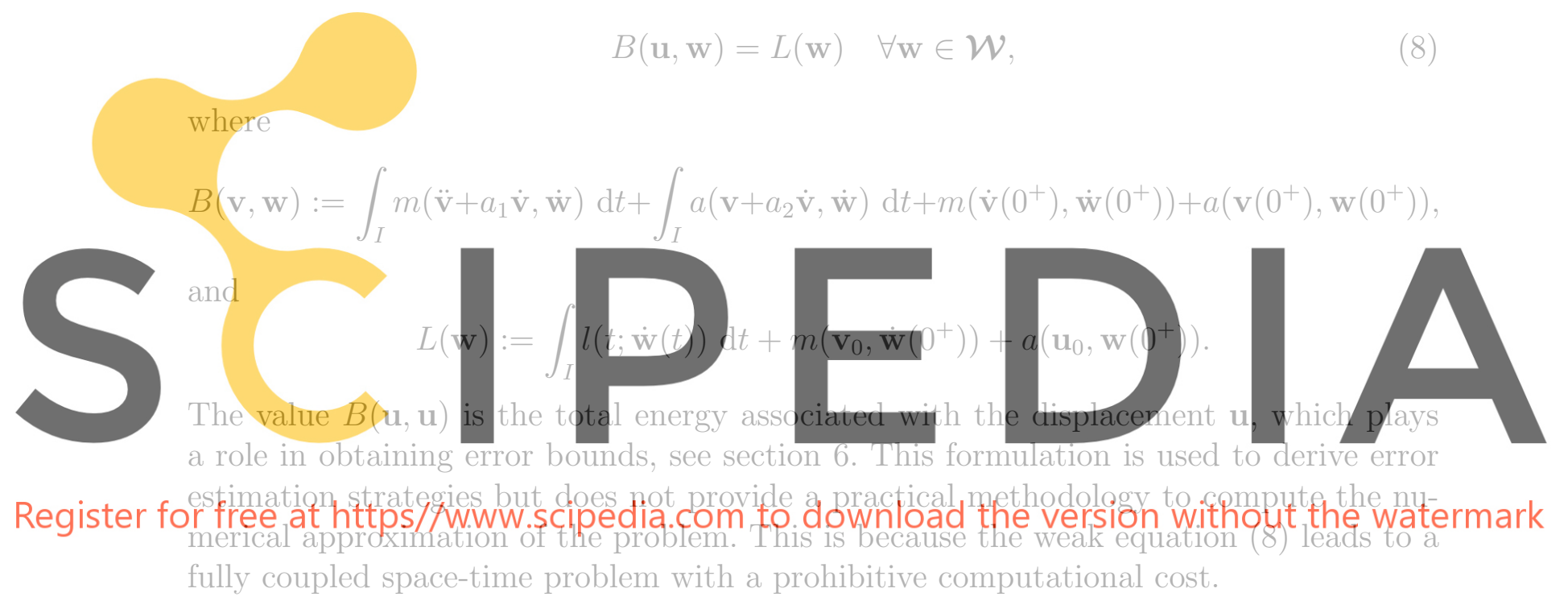

An usual alternative in transient elastodynamics is using Double field (or mixed) formulations, which introduce the velocity $\dot{\mathbf{u}}$ as new unknown. Thus, the unknown is the double field function $\mathbf{U}:=\left[\mathbf{u}_{u}, \mathbf{u}_{v}\right]:=[\mathbf{u}, \dot{\mathbf{u}}]$. The main advantage of mixed formulations is that they allow alleviating the continuity requirements on the solution. Instead of $\mathbf{u} \in \mathcal{W}$, the solution is $\mathbf{U} \in \mathcal{W}_{0} \times \mathcal{W}_{0}$ where

$$
\mathcal{W}_{0}:=\left\{\mathbf{w} \in L^{2}\left(I ; \mathcal{V}_{0}\right) \text { with } \dot{\mathbf{w}} \in L^{2}\left(I ; \mathcal{V}_{0}^{\prime}\right)\right\}
$$

Note that, in particular, this implies that $\mathbf{u}_{u}$ and $\mathbf{u}_{v} \in C^{0}\left(\bar{I} ;\left[L^{2}(\Omega)\right]^{d}\right)$ but their derivatives are not necessarily continuous.

The trial space for the double field time-continuous and time-discontinuous Galerkin formulations is defined as

$$
\widehat{\mathcal{W}}:=\left\{\mathbf{w} \in L^{2}\left(I ; \mathcal{V}_{0}\right) \text { with }\left.\mathbf{w}\right|_{I_{n}} \in H^{1}\left(I_{n} ; \mathcal{V}_{0}\right), n=1, \ldots, N\right\}
$$

Note that functions in $\widehat{\mathcal{W}}$ may be time-discontinuous. This property is necessary to decouple the solution in successive time intervals $I_{n}$. 
With these notations, the double field time continuous Galerkin weak form of problem (1) presented in [35] reads: find $\mathbf{U} \in \mathcal{W}_{0} \times \mathcal{W}_{0}$ such that for all

$$
B_{\mathrm{CGM}}(\mathbf{U}, \mathbf{W})=L_{\mathrm{CGM}}(\mathbf{W}) \quad \forall \mathbf{W} \in \widehat{\mathcal{W}} \times \widehat{\mathcal{W}}
$$

where $B_{\mathrm{CGM}}(\cdot, \cdot)$ and $L_{\mathrm{CGM}}(\cdot)$ are defined as

$$
\begin{aligned}
B_{\mathrm{CGM}}(\mathbf{U}, \mathbf{W}) & :=\int_{I} m\left(\dot{\mathbf{u}}_{v}+a_{1} \mathbf{u}_{v}, \mathbf{w}_{v}\right) \mathrm{d} t+\int_{I} a\left(\mathbf{u}_{u}+a_{2} \mathbf{u}_{v}, \mathbf{w}_{v}\right) \mathrm{d} t+m\left(\mathbf{u}_{v}\left(0^{+}\right), \mathbf{w}_{v}\left(0^{+}\right)\right) \\
& +\int_{I} m\left(\dot{\mathbf{u}}_{u}-\mathbf{u}_{v}, \mathbf{w}_{u}\right) \mathrm{d} t+m\left(\mathbf{u}_{u}\left(0^{+}\right), \mathbf{w}_{u}\left(0^{+}\right)\right), \\
L_{\mathrm{CGM}}(\mathbf{W}) & :=\int_{I} l\left(t ; \mathbf{w}_{v}\right) \mathrm{d} t+m\left(\mathbf{u}_{0}, \mathbf{w}_{u}\left(0^{+}\right)\right)+m\left(\mathbf{v}_{0}, \mathbf{w}_{v}\left(0^{+}\right)\right),
\end{aligned}
$$

and the general notation $\mathrm{W}:=\left[\mathrm{W}_{u}, \mathrm{~W}_{v}\right]$ is used. Note that the constrain $\dot{\mathrm{V}}_{u}=\mathrm{V}_{v}$ is weakly enforced using the mass product defined by $m(\cdot, \cdot)$ and this is the reason of using $\mathrm{M}$ in the notation for this approach.

A fully discrete solution is obtained replacing the infinite dimensional spaces involved in the weak form (9) by the discrete spaces
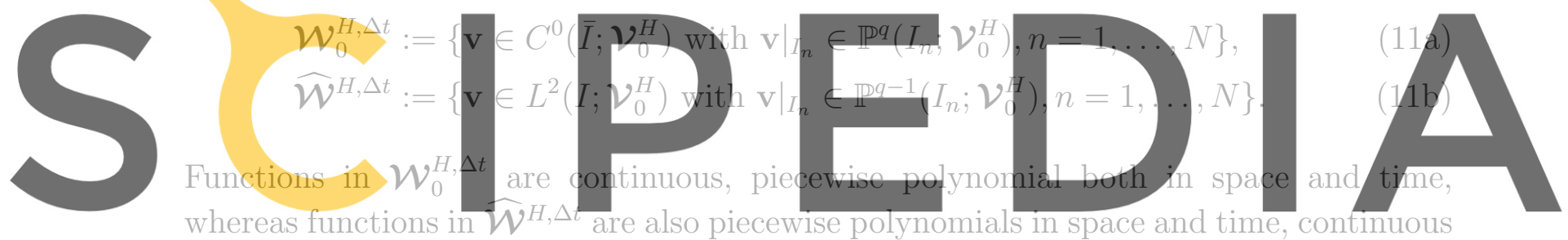

whereas functions in $\mathcal{W}^{H, \Delta t}$ are also piecewise polynomials in space and time, continuous

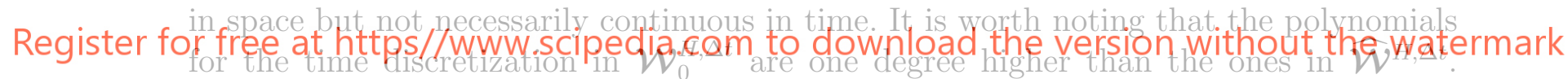

However, properly accounting for the initial conditions, the dimensions of $\mathcal{W}_{0}^{H, \Delta t}$ and $\widehat{\mathcal{W}}^{H, \Delta t}$ coincide due to the continuity requirements of $\mathcal{W}_{0}^{H, \Delta t}$.

The fully discrete equation reads: find $\widetilde{\mathbf{U}}:=\left[\tilde{\mathbf{u}}_{u}, \tilde{\mathbf{u}}_{v}\right] \in \mathcal{W}_{0}^{H, \Delta t} \times \mathcal{W}_{0}^{H, \Delta t}$ such that

$$
B_{\mathrm{CGM}}(\widetilde{\mathbf{U}}, \mathbf{W})=L_{\mathrm{CGM}}(\mathbf{W}) \quad \forall \mathbf{W} \in \widehat{\mathcal{W}}^{H, \Delta t} \times \widehat{\mathcal{W}}^{H, \Delta t}
$$

As mentioned before, although problem (12) is integrated over the whole space-time domain $\Omega \times I$, the discontinuities of the test functions allow decoupling the problem into $N$ problems posed over the time slabs $\Omega \times I_{n}, n=1, \ldots, N$. To be more precise, $\widetilde{\mathbf{U}}$ is computed recursively starting from $I_{1}$ and going forward in time (from $n=1$ to $N)$. In each time slab, $\left.\widetilde{\mathbf{U}}\right|_{I_{n}} \in \mathbb{P}^{q}\left(I_{n} ; \mathcal{V}_{0}^{H}\right) \times \mathbb{P}^{q}\left(I_{n} ; \mathcal{V}_{0}^{H}\right)$ is the solution of (12) where the function $\mathbf{W}$ is restricted to $I_{n}$ (with a zero value outside the time slab). The unknown $\left.\widetilde{\mathbf{U}}\right|_{I_{n}}$ accounts for the initial conditions given by the solution at the end point of the previous time-slab, $\left.\widetilde{\mathbf{U}}\right|_{I_{n}}\left(t_{n-1}^{+}\right)=\left.\widetilde{\mathbf{U}}\right|_{I_{n-1}}\left(t_{n-1}^{-}\right)=\left[\tilde{\mathbf{u}}_{u}\left(t_{n-1}^{-}\right), \tilde{\mathbf{u}}_{v}\left(t_{n-1}^{-}\right)\right]$(or $\left[\mathbf{u}_{0}, \mathbf{v}_{0}\right]$ for the first slab). In general, this method requires solving for each time step $2 q$ coupled spatial problems in $\mathcal{V}_{0}^{H}$. Recall that the Newmark method requires a single problem in $\mathcal{V}_{0}^{H}$ at each time step. However, for $q=1$ the block system of algebraic linear equations of 
double size can be pre-processed to make it equivalent to the Newmark method with parameters $\beta=1 / 4$ and $\gamma=1 / 2$, see [35] for a detailed proof.

Eriksson et al. [50] consider an alternative energy consistent weak form analogous to (9), where the velocity-displacement compatibility and initial conditions for the displacements are enforced using the energy product $a(\cdot, \cdot)$. The weak formulation reads: find $\mathbf{U} \in \mathcal{W}_{0} \times \mathcal{W}_{0}$ such that

$$
B_{\mathrm{CGA}}(\mathbf{U}, \mathbf{W})=L_{\mathrm{CGA}}(\mathbf{W}) \quad \forall \mathbf{W} \in \widehat{\mathcal{W}} \times \widehat{\mathcal{W}}
$$

where

$$
\begin{aligned}
B_{\mathrm{CGA}}(\mathbf{U}, \mathbf{W}) & :=\int_{I} m\left(\dot{\mathbf{u}}_{v}+a_{1} \mathbf{u}_{v}, \mathbf{w}_{v}\right) \mathrm{d} t+\int_{I} a\left(\mathbf{u}_{u}+a_{2} \mathbf{u}_{v}, \mathbf{w}_{v}\right) \mathrm{d} t+m\left(\mathbf{u}_{v}\left(0^{+}\right), \mathbf{w}_{v}\left(0^{+}\right)\right) \\
& +\int_{I} a\left(\dot{\mathbf{u}}_{u}-\mathbf{u}_{v}, \mathbf{w}_{u}\right) \mathrm{d} t+a\left(\mathbf{u}_{u}\left(0^{+}\right), \mathbf{w}_{u}\left(0^{+}\right)\right) \\
L_{\mathrm{CGA}}(\mathbf{W}) & :=\int_{I} l\left(t ; \mathrm{w}_{v}\right) \mathrm{d} t+a\left(\mathbf{u}_{0}, \mathrm{w}_{u}\left(0^{+}\right)\right)+m\left(\mathbf{v}_{0}, \mathrm{w}_{v}\left(0^{+}\right)\right) .
\end{aligned}
$$

It is easily seen that the bilinear form $B_{\mathrm{CGA}}(\cdot, \cdot)$ is energy consistent, namely $B_{\mathrm{CGA}}(\mathbf{U}, \mathbf{U})=B(\mathbf{u}, \mathbf{u})$. Note that the previous CGM variational formulation does not fulfill this property, that is $B_{\mathrm{CGM}}(\mathbf{U}, \mathbf{U}) \neq B(\mathbf{u}, \mathbf{u} \mathbf{)}$. This is due to the fact that for CGM
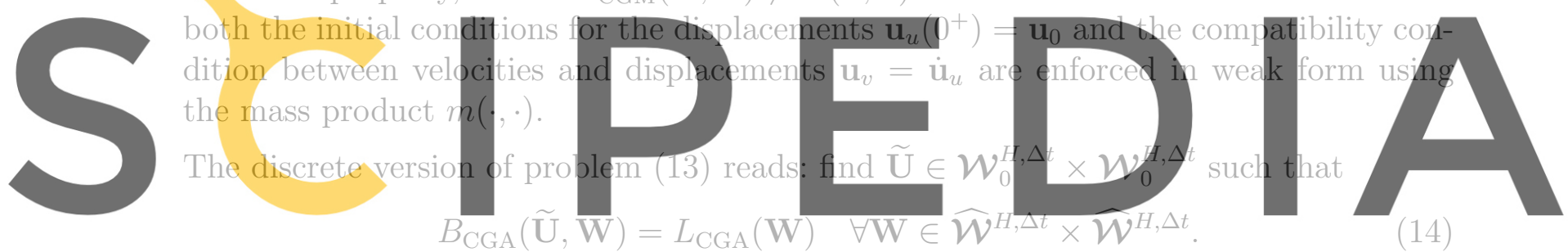

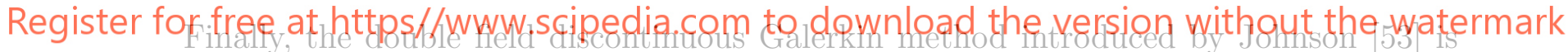

presented. This approach is a variant of the weak problem (13) in which both the trial and test functions are allowed to be discontinuous at time points in $\mathcal{T}$.

The continuity of the solution is weakly imposed by adding extra terms to the variational formulation, penalizing the time jumps of the solution at $\mathcal{T}$. The time discontinuous Galerkin weak form reads: find $\mathbf{U} \in \widehat{\mathcal{W}} \times \widehat{\mathcal{W}}$ such that

$$
B_{\mathrm{DG}}(\mathbf{U}, \mathbf{W})=L_{\mathrm{CGA}}(\mathbf{W}) \quad \forall \mathbf{W} \in \widehat{\mathcal{W}} \times \widehat{\mathcal{W}},
$$

where

$$
\begin{aligned}
B_{\mathrm{DG}}(\mathbf{U}, \mathbf{W}) & :=\sum_{n=1}^{N} \int_{I_{n}}\left(m\left(\dot{\mathbf{u}}_{v}+a_{1} \mathbf{u}_{v}, \mathbf{w}_{v}\right)+a\left(\mathbf{u}_{u}+a_{2} \mathbf{u}_{v}, \mathbf{w}_{v}\right)\right) \mathrm{d} t+m\left(\mathbf{u}_{v}\left(0^{+}\right), \mathbf{w}_{v}\left(0^{+}\right)\right) \\
& +\sum_{n=1}^{N} \int_{I_{n}} a\left(\dot{\mathbf{u}}_{u}-\mathbf{u}_{v}, \mathbf{w}_{u}\right) \mathrm{d} t+a\left(\mathbf{u}_{u}\left(0^{+}\right), \mathbf{w}_{u}\left(0^{+}\right)\right) \\
& +\sum_{n=1}^{N-1} m\left(\mathbf{u}_{v}\left(t_{n}^{+}\right)-\mathbf{u}_{v}\left(t_{n}^{-}\right), \mathbf{w}_{v}\left(t_{n}^{+}\right)\right)+\sum_{n=1}^{N-1} a\left(\mathbf{u}_{v}\left(t_{n}^{+}\right)-\mathbf{u}_{u}\left(t_{n}^{-}\right), \mathbf{w}_{u}\left(t_{n}^{+}\right)\right) .
\end{aligned}
$$


Note that, in order to obtain an energy consistent bilinear form, the jumps of the velocities and displacements at time $t_{n}, \mathbf{u}_{v}\left(t_{n}^{+}\right)-\mathbf{u}_{v}\left(t_{n}^{-}\right)$and $\mathbf{u}_{u}\left(t_{n}^{+}\right)-\mathbf{u}_{u}\left(t_{n}^{-}\right)$respectively, are introduced differently in the formulation. The mass bilinear form $m(\cdot, \cdot)$ is used for velocities whereas the energy product $a(\cdot, \cdot)$ is used for displacements, in such a way that $B_{\mathrm{DG}}(\mathbf{U}, \mathbf{U})=B(\mathbf{u}, \mathbf{u})$.

The discrete version of problem (15) is obtained replacing the space $\widehat{\mathcal{W}}$ by the discrete space $\widehat{\mathcal{W}}^{H, \Delta t}$ defined in equation (11b), namely: find $\widehat{\mathbf{U}}:=\left[\hat{\mathbf{u}}_{u}, \hat{\mathbf{u}}_{v}\right] \in \widehat{\mathcal{W}}^{H, \Delta t} \times \widehat{\mathcal{W}}^{H, \Delta t}$ such that

$$
B_{\mathrm{DG}}(\widehat{\mathbf{U}}, \mathbf{W})=L_{\mathrm{CGA}}(\mathbf{W}) \quad \forall \mathbf{W} \in \widehat{\mathcal{W}}^{H, \Delta t} \times \widehat{\mathcal{W}}^{H, \Delta t}
$$

The discrete problem (16) leads to $N$ uncoupled local problems posed over the time slabs $\Omega \times I_{n}, n=1, \ldots, N$. As in the previous double field formulations, the solution is computed recursively starting from $I_{1}$ and going forward in time. This approach requires solving, in each time slab, $2(q+1)$ coupled spatial problems in $\mathcal{V}_{0}^{H}$. Thus, for $q=1$ the dimension of the linear system to be solved in each time slab is four times larger than the system to be solved with the Newmark method. An efficient resolution strategy for this problem is presented in [29].

The error estimation techniques described in the forthcoming sections are presented using the previous variational formulations. Specifically, the recovery estimates described in section 4 consider the double field discontinuous Galerkin formulation (15), the explicit estimates and dual weighted residual technique described in section 5 are based on the double field time-continuous formulations (9) and (13) and finally, the implicit error estimates presented in section 6 require deriving error representations based on the standard single-field time-continuous Galerkin method (8).

\section{Error measures and error representation}

\subsection{Errors and error equations}

The error associated with a single field numerical approximation $\tilde{\mathbf{u}} \approx \mathbf{u}$, for instance the one introduced in (7), is defined as

$$
\mathbf{e}:=\mathbf{u}-\tilde{\mathbf{u}} \in \mathcal{W}
$$

Function e fulfills the following residual equation: find $\mathbf{e} \in \mathcal{W}$

$$
B(\mathbf{e}, \mathbf{w})=L(\mathbf{w})-B(\tilde{\mathbf{u}}, \mathbf{w})=: R(\mathbf{w}) .
$$

Equation (18) is derived replacing the exact solution $\mathbf{u}$ by $\tilde{\mathbf{u}}+\mathbf{e}$ into (8) and using the linearity of forms $B(\cdot, \cdot)$ and $L(\cdot)$. Note that, the residual $R(\cdot)$ is well defined only if the numerical approximation $\tilde{\mathbf{u}}$ is regular enough, that is $\tilde{\mathbf{u}} \in \mathcal{W}$. Thus, error techniques making use of the residual equation (18), in particular those presented in section 6, require that $\tilde{\mathbf{u}} \in \mathcal{W}$. 
In the case of using a double field formulation as (12) or (14), the numerical solution has the form $\widetilde{\mathbf{U}}=\left[\tilde{\mathbf{u}}_{u}, \tilde{\mathbf{u}}_{v}\right] \in \mathcal{W}_{0}^{H, \Delta t} \times \mathcal{W}_{0}^{H, \Delta t}$ and the error is defined by

$$
\mathbf{E}:=\left[\mathbf{e}_{u}, \mathbf{e}_{v}\right]:=\left[\mathbf{u}-\tilde{\mathbf{u}}_{u}, \dot{\mathbf{u}}-\tilde{\mathbf{u}}_{v}\right] \in \mathcal{W}_{0} \times \mathcal{W}_{0}
$$

where $\mathbf{e}_{u}$ and $\mathbf{e}_{v}$ are the errors in displacements and velocities respectively. Here, two different residual equations for the double field error $\mathbf{E}$ are derived replacing the exact solution $\mathbf{U}$ by $\widetilde{\mathbf{U}}+\mathbf{E}$ either into equation (9) or (13). That is, the error $\mathbf{E} \in \mathcal{W}_{0} \times \mathcal{W}_{0}$ is the solution of both

$$
B_{\mathrm{CGM}}(\mathbf{E}, \mathbf{W})=L_{\mathrm{CGM}}(\mathbf{W})-B_{\mathrm{CGM}}(\widetilde{\mathbf{U}}, \mathbf{W})=: R_{\mathrm{CGM}}(\mathbf{W}) \quad \forall \mathbf{W} \in \mathcal{W}_{0} \times \mathcal{W}_{0}
$$

and

$$
B_{\mathrm{CGA}}(\mathbf{E}, \mathbf{W})=L_{\mathrm{CGA}}(\mathbf{W})-B_{\mathrm{CGA}}(\widetilde{\mathbf{U}}, \mathbf{W})=: R_{\mathrm{CGA}}(\mathbf{W}) \quad \forall \mathbf{W} \in \mathcal{W}_{0} \times \mathcal{W}_{0}
$$

Note that the previous two equations have the same solution, which is precisely $\mathbf{E}$. In practice, the criterion for selecting either equation (19) or (20) depends on whether the Galerkin orthogonality property holds or not. This is because in the error estimation procedures presented in section 5, Galerkin orthogonality is required to properly split the time and space error contributions.

Thus, if the numerical approximation $\widetilde{\mathbf{U}}$ is the solution of the discrete problem (12), the error estimation strategy utilizes equation (19) since, in this case, the following Galerkin orthogonality property holds,

$$
R_{\mathrm{CGM}}(\mathbf{W})=0 \text { for all } \mathbf{W} \in \widehat{\mathcal{W}}^{H, \Delta t} \times \widehat{\mathcal{W}}^{H, \Delta t} .
$$

Analogously, if the numerical approximation $\widetilde{\mathbf{U}}$ is solution of the discrete problem (14), then the error estimation strategy takes equation (20) because

$$
R_{\mathrm{CGA}}(\mathbf{W})=0 \text { for all } \mathbf{W} \in \widehat{\mathcal{W}}^{H, \Delta t} \times \widehat{\mathcal{W}}^{H, \Delta t}
$$

If the numerical approximation is computed using other techniques, like the Newmark method, both residual equations could be used for error estimation, but the error estimation technique could not rely on Galerkin orthogonality.

The double field formulation (15) could also be used to derive a residual equation for the double field error E. However, in the remainder of the paper this formulation is only used for recovery type estimates (which do not utilize a residual equation).

\subsection{Energy measures}

As previously mentioned, the bilinear form $B(\cdot, \cdot)$ induces an energy measure in elastodynamics. For the sake of providing a physical interpretation, the energy norm $B(\mathbf{u}, \mathbf{u})$ reads as follows:

$$
B(\mathbf{u}, \mathbf{u})=\|\mathbf{u}\|^{2}+\frac{1}{2}\|\dot{\mathbf{u}}(T)\|_{m}^{2}+\frac{1}{2}\|\mathbf{u}(T)\|_{a}^{2}+\frac{1}{2}\left\|\mathbf{v}_{0}\right\|_{m}^{2}+\frac{1}{2}\left\|\mathbf{u}_{0}\right\|_{a}^{2}
$$


where $\|\mathbf{v}\|_{m}^{2}:=m(\mathbf{v}, \mathbf{v})$ and $\|\mathbf{v}\|_{a}^{2}:=a(\mathbf{v}, \mathbf{v})$ are the squared norms induced by the bilinear forms $m(\cdot, \cdot)$ and $a(\cdot, \cdot)$, respectively, and the space-time norm $\|\cdot\| \|$ is defined as

$$
\|\mathbf{v}\|:=\left(\int_{I} a_{1}\|\dot{\mathbf{v}}\|_{m}^{2} \mathrm{~d} t+\int_{I} a_{2}\|\dot{\mathbf{v}}\|_{a}^{2} \mathrm{~d} t\right)^{1 / 2} .
$$

The terms $\frac{1}{2}\|\dot{\mathbf{u}}(T)\|_{m}^{2}$ and $\frac{1}{2}\|\mathbf{u}(T)\|_{a}^{2}$ are the kinetic and elastic energy of $\mathbf{u}$ at time $t=T$, while the term $\|\mathbf{u}\|^{2}$ stands for the dissipated energy between times $t=0$ and $t=T$ due to the presence of damping in the equations, introduced by $a_{1}$ and $a_{2}$.

The different terms in (22) are used in the following to measure the error. For instance, the recovery estimates presented in section 4 make use of the squared norm $\|\cdot\|_{m}^{2}+\|\cdot\|_{a}^{2}$. Note that this is the only relevant energy measure of the error in the case of elastodynamics (for $a_{1}=a_{2}=0$ the dissipated error is zero, $\|\mathbf{e}\|=0$ ). On the contrary, dissipation is crucial to derive error bounds, as it is shown in section 6. Actually, the techniques computing upper bounds yield estimates $\eta_{\text {ener }}$ such that $\|\mathbf{e}\| \leq \eta_{\text {ener }}$.

However, there are norms different than those appearing in (22) which are also used in the literature. As an example, reference [54] measures the error with an $L^{2}$ norm of the displacements at the final simulation time $T$.

\subsection{Quantities of interest and adjoint problem}

Information provided by global error estimates (based on global norms) is not sufficient to make engineering decisions. Alternatively, the end-user often prefers measuring the error using some specific Quantity of Interest (QoI), which are particular functional outputs of the solution.

The quantity of interest is defined by a functional $L^{\mathcal{O}}: \mathcal{W} \longrightarrow \mathbb{R}$ which extracts a single representative scalar value of the whole space-time solution. The value $L^{\mathcal{O}}(\mathbf{u})$ is the quantity of interest, which is approximated by $L^{\mathcal{O}}(\tilde{\mathbf{u}})$ given a numerical approximation of the solution $\tilde{\mathbf{u}} \approx \mathbf{u}$. Goal-oriented error estimation strategies aim at assessing the quality of $L^{\mathcal{O}}(\tilde{\mathbf{u}})$, that is, the difference between the exact quantity of interest $L^{\mathcal{O}}(\mathbf{u})$ and the approximated one $L^{\mathcal{O}}(\tilde{\mathbf{u}}), L^{\mathcal{O}}(\mathbf{u})-L^{\mathcal{O}}(\tilde{\mathbf{u}})$. In the remainder of the paper it is assumed that the functional $L^{\mathcal{O}}$ is linear. Thus, $L^{\mathcal{O}}(\mathbf{u})-L^{\mathcal{O}}(\tilde{\mathbf{u}})$ coincides with $L^{\mathcal{O}}(\mathbf{e})$. However, non-linear functionals can also be handled using the same strategies after a simple linearization, see $[35,47]$ for details.

The estimation of value $L^{\mathcal{O}}(\mathbf{e})$ requires introducing an auxiliary problem associated with functional $L^{\mathcal{O}}(\cdot)$, usually denoted by adjoint or dual problem [31, 47, 42, 43, 44, 45, 46]. The variational form of the adjoint problem consists in finding $\mathbf{u}^{\mathrm{d}} \in \mathcal{W}$ such that

$$
B\left(\mathbf{w}, \mathbf{u}^{\mathrm{d}}\right)=L^{\mathcal{O}}(\mathbf{w}) \quad \forall \mathbf{w} \in \mathcal{W} .
$$

The adjoint solution $\mathbf{u}^{\mathrm{d}}$ characterizes the quantity of interest defined by $L^{\mathcal{O}}(\cdot)$. Note that if $\mathbf{u}^{\mathrm{d}}$ is available, the computable quantity $L\left(\mathbf{u}^{\mathrm{d}}\right)$ is equal to the quantity of interest $L^{\mathcal{O}}(\mathbf{u})$. In that sense, $\mathbf{u}^{\mathrm{d}}$ can be seen as the Riesz representation of functional $L^{\mathcal{O}}(\cdot)$. 
In practice, $L^{\mathcal{O}}(\cdot)$ is selected with the same structure as the functional $L(\cdot)$, namely

$$
L^{\mathcal{O}}(\mathbf{w}):=\int_{0}^{T}\left(\mathbf{f}^{\mathcal{O}}(t), \dot{\mathbf{w}}(t)\right) \mathrm{d} t+\int_{0}^{T}\left(\mathbf{g}^{\mathcal{O}}(t), \dot{\mathbf{w}}(t)\right)_{\Gamma_{\mathrm{N}}} \mathrm{d} t+m\left(\mathbf{v}^{\mathcal{O}}, \dot{\mathbf{w}}(T)\right)+a\left(\mathbf{u}^{\mathcal{O}}, \mathbf{w}(T)\right),
$$

where $\mathbf{f}^{\mathcal{O}}, \mathbf{g}^{\mathcal{O}}, \mathbf{v}^{\mathcal{O}}$ and $\mathbf{u}^{\mathcal{O}}$ are the data characterizing the quantity of interest. The functions $\mathbf{f}^{\mathcal{O}}$ and $\mathbf{g}^{\mathcal{O}}$ extract global or localized averages of velocities in $\Omega$ and $\Gamma_{\mathrm{N}}$, respectively, over the whole time interval $[0, T]$. The fields $\mathbf{v}^{\mathcal{O}}$ and $\mathbf{u}^{\mathcal{O}}$ play the role of weighting functions to compute averages of velocities and strains at the final simulation time $T$.

In this case, the associated strong form of the adjoint problem is

$$
\begin{aligned}
\rho\left(\ddot{\mathbf{u}}^{\mathrm{d}}-a_{1} \dot{\mathbf{u}}^{\mathrm{d}}\right)-\boldsymbol{\nabla} \cdot \boldsymbol{\sigma}^{\mathrm{d}} & =-\mathbf{f}^{\mathcal{O}} \quad \text { in } \Omega \times I, \\
\mathbf{u}^{\mathrm{d}} & =\mathbf{0} \quad \text { on } \Gamma_{\mathrm{D}} \times I, \\
\boldsymbol{\sigma}^{\mathrm{d}} \cdot \mathbf{n} & =-\mathbf{g}^{\mathcal{O}} \quad \text { on } \Gamma_{\mathrm{N}} \times I, \\
\mathbf{u}^{\mathrm{d}} & =\mathbf{u}^{\mathcal{O}} \quad \text { at } \Omega \times\{T\}, \\
\dot{\mathbf{u}}^{\mathrm{d}} & =\mathbf{v}^{\mathcal{O}} \quad \text { at } \Omega \times\{T\},
\end{aligned}
$$

with the constitutive law

$$
\boldsymbol{\sigma}^{\mathrm{d}}\left(\mathbf{u}^{\mathrm{d}}\right):=\mathcal{C}: \boldsymbol{\varepsilon}\left(\mathbf{u}^{\mathrm{d}}-a_{2} \dot{\mathbf{u}}^{\mathrm{d}}\right) .
$$

Note that the terms affected by $a_{1}$ and $a_{2}$ have opposite sign that the ones in the original problem (1). Consequently, the adjoint problem has exactly the same structure as the original (1) if integrated backwards in time starting from the final conditions (26d) and (26e).

Having selected the format of the quantity of interest given in (25) yields the adjoint problem (24) analogous to the original one (1). Thus, the same computer code available for solving the original problem (1) can be reused to solve the adjoint problem (26).

Remark 2. Note that the functional $L^{\mathcal{O}}(\cdot)$ as defined in (25) does not directly allow to compute averages of the displacements of $\mathbf{u}$ over the time interval $I=(0, T]$, namely

$$
\int_{I}(\boldsymbol{\lambda}(t), \mathbf{u}(t)) \mathrm{d} t
$$

However, it is easy to see that it is possible to express this quantity as

$$
\int_{I}(\boldsymbol{\lambda}(t), \mathbf{u}(t)) \mathrm{d} t=\int_{0}^{T}\left(\mathbf{f}^{\mathcal{O}}(t), \dot{\mathbf{u}}(t)\right) \mathrm{d} t-\left(\mathbf{f}^{\mathcal{O}}(0), \mathbf{u}_{0}\right),
$$

for

$$
\mathbf{f}^{\mathcal{O}}(t)=\int_{t}^{T} \boldsymbol{\lambda}(\xi) \mathrm{d} \xi
$$

Since the term $\left(\mathbf{f}^{\mathcal{O}}(0), \mathbf{u}_{0}\right)$ is constant, assessing the error in the quantity of interest (28) is equivalent to assess $L^{\mathcal{O}}(\mathbf{e})$ where the data characterizing $L^{\mathcal{O}}(\cdot)$ are $\mathbf{g}^{\mathcal{O}}=\mathbf{v}^{\mathcal{O}}=\mathbf{u}^{\mathcal{O}}=\mathbf{0}$ and $\mathbf{f}^{\mathcal{O}}$ defined in equation (29). 
Similarly, the average of displacements at the final time of the computation

$$
\left(\boldsymbol{\lambda}^{\mathcal{O}}, \mathbf{u}(T)\right)+\left(\boldsymbol{\lambda}_{N}^{\mathcal{O}}, \mathbf{u}(T)\right)_{\Gamma_{N}}
$$

where the data $\boldsymbol{\lambda}^{\mathcal{O}}$ and $\boldsymbol{\lambda}_{N}^{\mathcal{O}}$ are weighting functions allowing to localize the average of displacements in some sub-domains in $\Omega$ and $\Gamma_{\mathrm{N}}$ respectively, is neither directly included in (25). In this case, an auxiliary problem is introduced: find $\mathbf{u}^{\mathcal{O}} \in \mathcal{V}_{0}$ solution of the static problem

$$
a\left(\mathbf{u}^{\mathcal{O}}, \mathbf{w}\right)=\left(\boldsymbol{\lambda}^{\mathcal{O}}, \mathbf{w}\right)+\left(\boldsymbol{\lambda}_{N}^{\mathcal{O}}, \mathbf{w}\right)_{\Gamma_{N}} \quad \forall \mathbf{w} \in \mathcal{V}_{0}
$$

Note that here $\mathbf{u}^{\mathcal{O}}$ is not given as part of the data $\boldsymbol{\lambda}^{\mathcal{O}}$ and $\boldsymbol{\lambda}_{N}^{\mathcal{O}}$ characterizing (30). The function $\mathbf{u}^{\mathcal{O}}$ has to be computed as the solution of (31). Taking $\mathbf{w}=\mathbf{e}(T)$ in (31) it is easily seen that assessing the error in the quantity of interest (30) is equivalent to assess $L^{\mathcal{O}}(\mathbf{e})$ where in this case the data characterizing $L^{\mathcal{O}}(\cdot)$ are $\mathbf{f}^{\mathcal{O}}=\mathbf{g}^{\mathcal{O}}=\mathbf{v}^{\mathcal{O}}=\mathbf{0}$ and $\mathbf{u}^{\mathcal{O}}$ defined in equation (31).

Remark 3 (Illustrative example). The following example illustrates the adjoint problem given in (26) for a one dimensional example. The spatial computational domain is $\Omega=$ $(0,1) \mathrm{m}$, the boundaries are $\Gamma_{\mathrm{N}}=\{0 \mathrm{~m}\}$ and $\Gamma_{\mathrm{D}}=\{1 \mathrm{~m}\}$, and the time interval is $I=$ $(0,2] \mathrm{s}$. The material properties are $E=1 \mathrm{~Pa}, \nu=0, \rho=1, \mathrm{~kg} / \mathrm{m}^{3}$ and $a_{1}=a_{2}=0 \mathrm{~s}$.

Two different adjoint problems are illustrated in this remark, associated with the quantities of interest

$$
L_{1}^{\mathcal{O}}(w)=\int_{I} \int_{\Omega} \alpha(t) \beta(x) \dot{w}(x, t) \mathrm{d} x \mathrm{~d} t \quad \text { and } L_{2}^{\mathcal{O}}(w)=\int_{I} \int_{\Omega} \alpha(t) \beta(x) w(x, t) \mathrm{d} x \mathrm{~d} t,
$$

where $\alpha(t)$ and $\beta(x)$ are the time dependent functions defined in figure 1. Note that
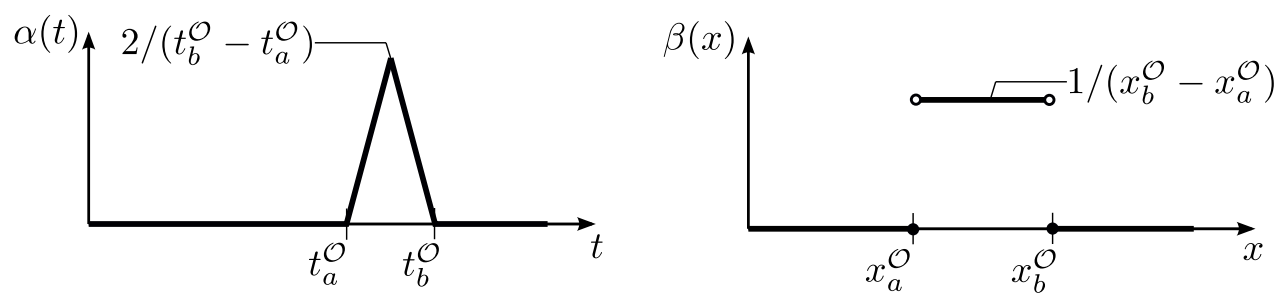

Figure 1: Definition of $\alpha(t)$ and $\beta(x)$, (left) and (right) respectively.

$L_{1}^{\mathcal{O}}(\cdot)$ corresponds to take $\mathbf{g}^{\mathcal{O}}=\mathbf{v}^{\mathcal{O}}=\mathbf{u}^{\mathcal{O}}=\mathbf{0}$ and $\mathbf{f}^{\mathcal{O}}=\alpha(t) \beta(x)$ in equation (25) and provides a weighted average of velocities in the space-time region $S^{\mathcal{O}}=\left(x_{a}^{\mathcal{O}}, x_{b}^{\mathcal{O}}\right) \times\left(t_{a}^{\mathcal{O}}, t_{b}^{\mathcal{O}}\right)$. On the other hand, $L_{2}^{\mathcal{O}}(\cdot)$ corresponds to take $\boldsymbol{\lambda}=\alpha(t) \beta(x)$ in (28) and provides a weighted average of displacement in $S^{\mathcal{O}}$. In this example, the region $S^{\mathcal{O}}$ is characterized by $x_{a}^{\mathcal{O}}=0.2 \mathrm{~m} x_{b}^{\mathcal{O}}=0.3 \mathrm{~m}, t_{a}^{\mathcal{O}}=1.8 \mathrm{~s}$ and $t_{b}^{\mathcal{O}}=1.9 \mathrm{~s}$.

The adjoint problems associated with quantities $L_{1}^{\mathcal{O}}$ and $L_{2}^{\mathcal{O}}$ are plotted in figure 2. Note that, the adjoint solutions are indeed the region of influence of each quantity of interest. That is, any perturbation taking place where the adjoint solution is zero has no influence in the quantity of interest. Note also that, the influence regions are different for quantities $L_{1}^{\mathcal{O}}$ and $L_{2}^{\mathcal{O}}$ even though they provide information of the solution $\mathbf{u}$ in the same space-time region $S^{\mathcal{O}}$. 

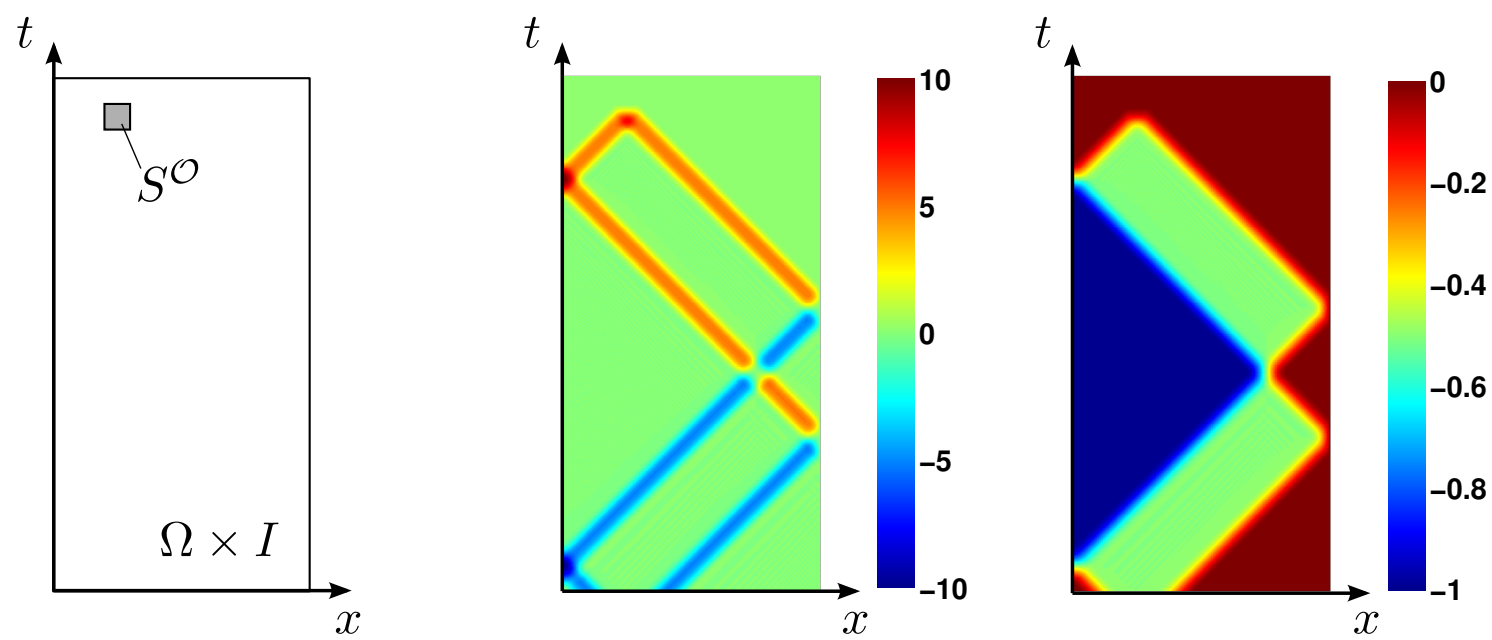

Figure 2: Illustration of the adjoint problem for two quantities of interest $L_{1}^{\mathcal{O}}$ and $L_{2}^{\mathcal{O}}$ (average of velocities and displacements in the region $S^{\mathcal{O}}$, respectively). Definition of the space-time domain $\Omega \times I$ and region of interest $S^{\mathcal{O}}$ (left). Adjoint velocities $[\mathrm{m} / \mathrm{s}]$ for quantity $L_{1}^{\mathcal{O}}$ (center). Adjoint velocities $[\mathrm{m} / \mathrm{s}]$ for quantity $L_{2}^{\mathcal{O}}$ (right).

The definition of the adjoint problem given in (24) depends on the weak form selected for the direct problem. Thus, the adjoint problems associated with the double field formulations CGM and CGA presented in (9) and (13) are introduced in the following. Note that the regularity restrictions for these problems are weaker than those of the previous adjoint problem. The adjoin problem associated with the formulation denoted as DG is not presented because, as previously said, this formulation is only used in the context of recovery type estimates for energy error measures.

The quantity of interest of a double field function $\mathbf{W}=\left[\mathbf{w}_{u}, \mathbf{w}_{v}\right] \in \mathcal{W}_{0} \times \mathcal{W}_{0}$ is defined as

$$
L_{\mathrm{D}}^{\mathcal{O}}(\mathbf{W}):=L_{u}^{\mathcal{O}}\left(\mathbf{w}_{u}\right)+L_{v}^{\mathcal{O}}\left(\mathbf{w}_{v}\right),
$$

where $L_{v}^{\mathcal{O}}: \mathcal{W}_{0} \longrightarrow \mathbb{R}$ and $L_{u}^{\mathcal{O}}: \mathcal{W}_{0} \longrightarrow \mathbb{R}$ are linear functionals extracting quantities of interest from velocities and displacements respectively. The variational form of the adjoint problem associated with a double field quantity $L_{\mathrm{D}}^{\mathcal{O}}(\cdot)$ is introduced for both formulations CGM and CGA given in section 2.2.2. Specifically, if the variational formulation CGM presented in (9) is considered, then, the adjoint problem reads: find $\mathbf{U}^{\mathrm{d}}:=\left[\mathbf{u}_{u}^{\mathrm{d}}, \mathbf{u}_{v}^{\mathrm{d}}\right] \in \mathcal{W}_{0} \times \mathcal{W}_{0}$ such that

$$
B_{\mathrm{CGM}}\left(\mathbf{W}, \mathbf{U}^{\mathrm{d}}\right)=L_{\mathrm{D}}^{\mathcal{O}}(\mathbf{W}) \quad \forall \mathbf{W} \in \mathcal{W}_{0} \times \mathcal{W}_{0},
$$

while considering the variational formulation CGA of (13) yields: find $\mathbf{U}^{\mathrm{d}} \in \mathcal{W}_{0} \times \mathcal{W}_{0}$ such that

$$
B_{\mathrm{CGA}}\left(\mathbf{W}, \mathbf{U}^{\mathrm{d}}\right)=L_{\mathrm{D}}^{\mathcal{O}}(\mathbf{W}) \quad \forall \mathbf{W} \in \mathcal{W}_{0} \times \mathcal{W}_{0}
$$

As discussed for the single field quantities of interest, in many practical applications it is important that the adjoint variational problem admits a strong form representation like the one given in (26). This introduces restrictions to the form of the linear functionals describing the quantities of interest as given in (32). 
Equation (33) leads to the strong equations (26) for functionals $L_{u}^{\mathcal{O}}(\cdot)$ and $L_{v}^{\mathcal{O}}(\cdot)$ defined as

$$
\begin{aligned}
& L_{u}^{\mathcal{O}}\left(\mathbf{w}_{u}\right):=m\left(\mathbf{u}^{\mathcal{O}}, \mathbf{w}_{u}(T)\right) \text { and } \\
& L_{v}^{\mathcal{O}}\left(\mathbf{w}_{v}\right):=\int_{0}^{T}\left(\mathbf{f}^{\mathcal{O}}(t), \mathbf{w}_{v}(t)\right) \mathrm{d} t+\int_{0}^{T}\left(\mathbf{g}^{\mathcal{O}}(t), \mathbf{w}_{v}(t)\right)_{\Gamma_{\mathrm{N}}} \mathrm{d} t+m\left(\mathbf{v}^{\mathcal{O}}, \mathbf{w}_{v}(T)\right),
\end{aligned}
$$

while equation (33) leads to the strong problem (26) for

$$
\begin{aligned}
& L_{u}^{\mathcal{O}}\left(\mathbf{w}_{u}\right):=a\left(\mathbf{u}^{\mathcal{O}}, \mathbf{w}_{u}(T)\right) \text { and } \\
& L_{v}^{\mathcal{O}}\left(\mathbf{w}_{v}\right):=\int_{0}^{T}\left(\mathbf{f}^{\mathcal{O}}(t), \mathbf{w}_{v}(t)\right) \mathrm{d} t+\int_{0}^{T}\left(\mathbf{g}^{\mathcal{O}}(t), \mathbf{w}_{v}(t)\right)_{\Gamma_{\mathrm{N}}} \mathrm{d} t+m\left(\mathbf{v}^{\mathcal{O}}, \mathbf{w}_{v}(T)\right) .
\end{aligned}
$$

It is worth noting that the quantity of interest associated to the energy consistent double field formulation CGA is the equivalent to the single field quantity of interest defined in (25). This is because the definition of $L_{D}^{\mathcal{O}}$ in $(36)$ is such that $L_{D}^{\mathcal{O}}([\mathbf{w}, \dot{\mathbf{w}}])=L^{\mathcal{O}}(\mathbf{w})$.

\subsection{Error representation with adjoint problem}

The adjoint problem allows rewriting the error in the quantity of interest in terms of residuals, combining the original and adjoint problems. Thus, the error assessment for the quantity of interest is performed using standard error estimation techniques designed for global error measures. Different error representations for $L^{\mathcal{O}}(\mathbf{e})$, or similarly for $L_{\mathrm{D}}^{\mathcal{O}}(\mathbf{E})$ in the case of a double field formulation, are used for the error assessment strategies presented here.

Techniques aiming at furnishing bounds for the error in the quantity of interest, like the ones presented in section 6 , consider an error representation based on the adjoint weak residual, $R^{\mathrm{d}}(\cdot)$, associated with the numerical approximation of the adjoint problem $\tilde{\mathbf{u}}^{\mathrm{d}} \approx \mathbf{u}^{\mathrm{d}}$. This error representation reads

$$
L^{\mathcal{O}}(\mathbf{e})=R^{\mathrm{d}}(\mathbf{e})+B\left(\mathbf{e}, \tilde{\mathbf{u}}^{\mathrm{d}}\right)=R^{\mathrm{d}}(\mathbf{e})+R\left(\tilde{\mathbf{u}}^{\mathrm{d}}\right),
$$

where

$$
R^{\mathrm{d}}(\mathbf{w}):=L^{\mathcal{O}}(\mathbf{w})-B\left(\mathbf{w}, \tilde{\mathbf{u}}^{\mathrm{d}}\right) .
$$

Equation (37) is derived taking $\mathbf{w}=\mathbf{e}$ into (38) along with the definition of the primal residual. In equation (37), the error in the quantity of interest is expressed as the non-computable term $R^{\mathrm{d}}(\mathbf{e})$ plus the computable term $R\left(\tilde{\mathbf{u}}^{\mathrm{d}}\right)$. Thus, bounds for $L^{\mathcal{O}}(\mathbf{e})$ are obtained finding bounds for $R^{\mathrm{d}}(\mathbf{e})$. Note that, $R\left(\tilde{\mathbf{u}}^{\mathrm{d}}\right)$ is a computable quantity, not necessarily equal to zero. Therefore, numerical approximations $\tilde{\mathbf{u}}$ and $\tilde{\mathbf{u}}^{\mathrm{d}}$ are not assumed to fulfill any Galerkin orthogonality property. That allows using many different computational methodologies to obtain $\tilde{\mathbf{u}}$ and $\tilde{\mathbf{u}}^{\mathrm{d}}$. The only requirement is that the numerical solutions are regular enough, that is $\tilde{\mathbf{u}}, \tilde{\mathbf{u}}^{\mathrm{d}} \in \mathcal{W}$ and that the bounds for $R^{\mathrm{d}}(\mathbf{e})$ are available.

On the other hand, the error estimates presented in section 5 utilize a different error representation. In this case, the error in the quantity of interest is expressed using the 
primal residual and the exact adjoint solution. The following development is analogous for the CGM and the CGA formulations. For the sake of simplicity, the presentation is done for CGM but equivalent expressions stand replacing CGM by CGA. One of the simpler versions of the error representation reads

$$
L_{\mathrm{D}}^{\mathcal{O}}(\mathbf{E})=R_{\mathrm{CGM}}\left(\mathbf{U}^{\mathrm{d}}\right) .
$$

The previous equation is a direct consequence of the definition of both the residuals and the adjoint solution.

In the error estimation setup, replacing $\mathbf{U}^{\mathrm{d}}$ by an approximated value $\widetilde{\mathbf{U}}^{\mathrm{d}}$, the error representation (39) gives an accurate approximation of the error in the quantity of interest. However, the local error contributions provided by the local restrictions of this residual expression are often too pessimistic due to the cancellation effect of the contributions of opposite sign with large absolute values, see [55]. Thus, equation (39) is not used directly to compute error maps for space-time mesh adaptivity. The error representation (39) is modified adding and subtracting an arbitrary function $\mathbf{W}^{H, \Delta t} \in \mathcal{W}_{0}^{H, \Delta t} \times \mathcal{W}_{0}^{H, \Delta t}$ in the argument of the residual,

$$
L_{\mathrm{D}}^{\mathcal{O}}(\mathbf{E})=R_{\mathrm{CGM}}\left(\mathbf{U}^{\mathrm{d}}-\mathbf{W}^{H, \Delta t}\right)+R_{\mathrm{CGM}}\left(\mathbf{W}^{H, \Delta t}\right) .
$$

Then, using Galerkin orthogonality of the residuals it follows

$$
L_{\mathrm{D}}^{\mathcal{O}}(\mathbf{E})=R_{\mathrm{CGM}}\left(\mathbf{U}^{\mathrm{d}}-\mathbf{W}^{H, \Delta t}\right) .
$$

Globally, the error representation (41) is identical to (39). However, their local restrictions are different and, if $\mathbf{W}^{H, \Delta t}$ is properly selected, (41) provides a map of local error contributions better suited for adaptive purposes. In practice, the function $\mathbf{W}^{H, \Delta t}$ is taken as the projection onto the test space $\mathcal{W}_{0}^{H, \Delta t} \times \mathcal{W}_{0}^{H, \Delta t}$ of the computed adjoint approximation $\widetilde{\mathbf{U}}^{\mathrm{d}}$, see section 5 for details.

Note that the estimated error distribution given by representation (41) is valid only if Galerkin orthogonality holds. In practice it means that, if equation (41) is used, then $\widetilde{\mathbf{U}}$ must be the solution of the discrete problem (12).

\section{Recovery estimates}

In the following, recovery type error estimates in the framework of elastodynamics, see $[29,30,31]$, are applied to the DG formulations. That is, they assess the error with respect to the solution $\widehat{\mathbf{U}}$ of the discrete problem (16). Recovery type error estimates provide error indicators for the space and time components of the error, namely $\eta_{n}^{\mathrm{s}}$ and $\eta_{n}^{\mathrm{t}}$, associated with a point $t_{n}$ of the time discretization $\mathcal{T}$. These indicators are input data for an adaptive procedure, allowing to select the mesh size and time step and to design adapted discretizations. 


\subsection{Space-time error splitting}

For a given time $t_{n} \in \mathcal{T}$, the error associated with the DG approximation $\widehat{\mathbf{U}}=\left[\hat{\mathbf{u}}_{u}, \hat{\mathbf{u}}_{v}\right]$ is defined as

$$
\mathbf{E}\left(t_{n}\right):=\mathbf{U}\left(t_{n}\right)-\widehat{\mathbf{U}}\left(t_{n}^{-}\right)=\left[\mathbf{u}\left(t_{n}\right)-\hat{\mathbf{u}}_{u}\left(t_{n}^{-}\right), \dot{\mathbf{u}}\left(t_{n}\right)-\hat{\mathbf{u}}_{v}\left(t_{n}^{-}\right)\right] .
$$

Note that the error $\mathbf{E}\left(t_{n}\right)$ measures both the space and time discretization errors at time $t_{n}$. In order to separate the contribution of the space and time errors, the error $\mathbf{E}\left(t_{n}\right)$ is decomposed into

$$
\mathbf{E}\left(t_{n}\right)=\mathbf{E}^{\mathrm{S}}\left(t_{n}\right)+\mathbf{E}^{\mathrm{t}}\left(t_{n}\right),
$$

where $\mathbf{E}^{\mathrm{s}}\left(t_{n}\right)$ and $\mathbf{E}^{\mathrm{t}}\left(t_{n}\right)$ are defined as

$$
\mathbf{E}^{\mathrm{s}}\left(t_{n}\right):=\mathbf{U}\left(t_{n}\right)-\mathbf{U}^{H}\left(t_{n}\right) \text { and } \mathbf{E}^{\mathrm{t}}\left(t_{n}\right):=\mathbf{U}^{H}\left(t_{n}\right)-\widehat{\mathbf{U}}\left(t_{n}^{-}\right) .
$$

Note that $\mathbf{E}^{\mathrm{s}}\left(t_{n}\right)$ and $\mathbf{E}^{\mathrm{t}}\left(t_{n}\right)$ are defined introducing function $\mathbf{U}^{H}=\left[\mathbf{u}^{H}, \dot{\mathbf{u}}^{H}\right]$, being $\mathbf{u}^{H}$ the exact (in time) solution of the semidiscrete problem (4), which is unknown. Function $\mathbf{U}^{H}$ tends to the exact solution $\mathbf{U}$ as $H$ tends to zero. Consequently, also $\mathbf{E}^{\mathbf{s}}\left(t_{n}\right)$ tends to zero with $H$ and therefore it is referred as the space discretization error. Similarly, the term $\mathbf{E}^{\mathrm{t}}\left(t_{n}\right)$ tends to zero as the time step, used in the discretization of equation (4), tends to zero. Thus, $\mathbf{E}^{\mathrm{t}}\left(t_{n}\right)$ is associated with the error produced by the time discretization.

In order to derive local error indicators, the space and time discretization errors are measured using the kinetic and elastic energy. Note that the error measure used here corresponds to the total energy if the viscosity vanishes $\left(a_{1}=a_{2}=0\right)$ :

$$
\left\|\mathbf{E}^{\mathrm{s}}\left(t_{n}\right)\right\|_{m+a}^{2}:=\left\|\mathbf{e}_{v}^{\mathrm{s}}\left(t_{n}\right)\right\|_{m}^{2}+\left\|\mathbf{e}_{u}^{\mathrm{s}}\left(t_{n}\right)\right\|_{a}^{2} \quad \text { and } \quad\left\|\mathbf{E}^{\mathrm{t}}\left(t_{n}\right)\right\|_{m+a}^{2}:=\left\|\mathbf{e}_{v}^{\mathrm{t}}\left(t_{n}\right)\right\|_{m}^{2}+\left\|\mathbf{e}_{u}^{\mathrm{t}}\left(t_{n}\right)\right\|_{a}^{2} .
$$

The goal of recovery error estimates is to furnish error indicators $\eta_{n}^{\mathrm{s}}$ and $\eta_{n}^{\mathrm{t}}$ such that

$$
\eta_{n}^{\mathrm{s}} \approx\left\|\mathbf{E}^{\mathrm{s}}\left(t_{n}\right)\right\|_{m+a}^{2} \quad \text { and } \quad \eta_{n}^{\mathrm{t}} \approx\left\|\mathbf{E}^{\mathrm{t}}\left(t_{n}\right)\right\|_{m+a}^{2} .
$$

The time error indicators $\eta_{n}^{\mathrm{t}}, n=1, \ldots, N$ are directly used to define the desired size of the time step at each time $t_{n}$. On the other hand, the space error indicator $\eta_{n}^{\mathrm{s}}$ is decomposed into element contributions

$$
\eta_{n}^{\mathrm{s}}=\sum_{k=1}^{N_{\mathrm{el}}} \eta_{n, k}^{\mathrm{s}}
$$

where $\eta_{n, k}^{\mathrm{s}}$ is an estimate of the contribution of element $\Omega_{k}$ to the norm $\left\|\mathbf{E}^{\mathrm{s}}\left(t_{n}\right)\right\|_{m+a}^{2}$. The space error indicators $\eta_{n, k}^{\mathrm{s}}$ allow defining a desired element size in the zone of element $\Omega_{k}$ at time $t_{n}$. Thus, combining the information provided by $\eta_{n}^{\mathrm{t}}$ and $\eta_{n, k}^{\mathrm{s}}$, the adapted space and time discretizations are designed to meet the precision required.

The remainder of this section is devoted to the actual computation of the error indicators $\eta_{k, n}^{\mathrm{s}}$ and $\eta_{n}^{\mathrm{t}}$. 


\subsection{Assessing time discretization errors}

The local error indicators $\eta_{n}^{\mathrm{t}} \approx\left\|\mathbf{E}^{\mathrm{t}}\left(t_{n}\right)\right\|_{m+a}^{2}$ are computed in references [29, 30] using the time-discontinuities of the DG approximation $\widehat{\mathbf{U}}$, namely

$$
\eta_{n}^{\mathrm{t}}:=\left\|\widehat{\mathbf{U}}\left(t_{n}^{+}\right)-\widehat{\mathbf{U}}\left(t_{n}^{-}\right)\right\|_{m+a}=\left\|\hat{\mathbf{u}}_{v}\left(t_{n}^{+}\right)-\hat{\mathbf{u}}_{v}\left(t_{n}^{-}\right)\right\|_{m}^{2}+\left\|\hat{\mathbf{u}}_{u}\left(t_{n}^{+}\right)-\hat{\mathbf{u}}_{u}\left(t_{n}^{-}\right)\right\|_{a}^{2} .
$$

This definition is suggested by the super-convergent properties of the time-DG formulations, stating that the solution at $t_{n}^{-}$is much more accurate than the solution at $t_{n}^{+}$.

References $[56,57,31]$ introduce alternative indicators $\eta_{n}^{\mathrm{t}}$ if the numerical approximation is a time-continuous function $\widetilde{\mathbf{U}}$ instead of the DG approximation $\widehat{\mathbf{U}}$. In that, case the indicators cannot be computed using the time jumps and, therefore, the following alternative definition is introduced

$$
\eta_{n}^{\mathrm{t}}:=\left\|\mathbf{U}^{*}\left(t_{n}\right)-\tilde{\mathbf{U}}\left(t_{n}\right)\right\|_{m+a}^{2}=\left\|\mathbf{u}_{v}^{*}\left(t_{n}\right)-\tilde{\mathbf{u}}_{v}\left(t_{n}^{-}\right)\right\|_{m}^{2}+\left\|\mathbf{u}_{u}^{*}\left(t_{n}\right)-\tilde{\mathbf{u}}_{u}\left(t_{n}^{-}\right)\right\|_{a}^{2} .
$$

where $\mathbf{U}^{*}\left(t_{n}\right)$ is an enhanced (in time) function computed using the numerical approximation $\widetilde{\mathbf{U}}$ at previous time steps, see reference [31] for specific details. The post-processed solution $\mathbf{U}^{*}\left(t_{n}\right)$ replaces the exact (in time) approximation $\mathbf{U}^{H}\left(t_{n}\right)$ in order to obtain an error estimate.

\subsection{Assessing space discretization errors}

The natural post-process of the numerical solution for the recovery type error estimators, is performed for stress fields rather than for the displacements, see [3]. In this context, energy is measured in terms of stresses using the complementary energy norm $\|\cdot\|_{\bar{a}}$ defined as

$$
\|\boldsymbol{\sigma}\|_{\bar{a}}^{2}:=\bar{a}(\boldsymbol{\sigma}, \boldsymbol{\sigma}) \quad \text { where } \quad \bar{a}(\boldsymbol{\sigma}, \boldsymbol{\tau}):=\int_{\Omega} \boldsymbol{\sigma}: \mathcal{C}^{-1}: \boldsymbol{\tau} \mathrm{d} \Omega .
$$

Thus, value $\left\|\mathbf{E}^{\mathrm{s}}\left(t_{n}\right)\right\|_{m+a}^{2}$ is rewritten using $\|\cdot\|_{\bar{a}}$ as

$$
\left\|\mathbf{E}^{\mathrm{S}}\left(t_{n}\right)\right\|_{m+a}^{2}=\left\|\dot{\mathbf{u}}\left(t_{n}\right)-\dot{\mathbf{u}}^{H}\left(t_{n}\right)\right\|_{m}^{2}+\left\|\boldsymbol{\sigma}\left(\mathbf{u}\left(t_{n}\right)\right)-\boldsymbol{\sigma}\left(\mathbf{u}^{H}\left(t_{n}\right)\right)\right\|_{\bar{a}}^{2} .
$$

In the framework of space error indicators, the semi-discrete (exact in time) functions $\dot{\mathbf{u}}^{H}\left(t_{n}\right)$ and $\boldsymbol{\sigma}\left(\mathbf{u}^{H}\left(t_{n}\right)\right)$ are assumed to fairly approximate $\hat{\mathbf{u}}_{v}\left(t_{n}\right)$ and $\boldsymbol{\sigma}\left(\hat{\mathbf{u}}_{u}\left(t_{n}\right)\right)$. This is equivalent to assume that the time integration error of the numerical approximation $\widehat{\mathbf{U}}\left(t_{n}\right)=\left[\hat{\mathbf{u}}_{u}\left(t_{n}\right), \hat{\mathbf{u}}_{v}\left(t_{n}\right)\right]$ is small. This requires reducing the time discretization error before assessing the space discretization error. Thus, the adaptive strategy aims at reaching the prescribed time accuracy at each time $t_{n}$ before starting with the space adaptive procedure.

A computable value for $\left\|\mathbf{E}^{\mathrm{S}}\left(t_{n}\right)\right\|_{m+a}^{2}$ using equation (44) is obtained recovering approximations of the exact velocities $\mathbf{v}_{n}^{*} \approx \dot{\mathbf{u}}\left(t_{n}\right)$ and stresses $\boldsymbol{\sigma}_{n}^{*} \approx \boldsymbol{\sigma}\left(\mathbf{u}\left(t_{n}\right)\right)$ with some post-process of $\widehat{\mathbf{U}}\left(t_{n}\right)$. Introducing these approximations, the space error contributions $\eta_{n}^{\mathrm{s}} \approx\left\|\mathbf{E}^{\mathrm{s}}\left(t_{n}\right)\right\|_{m+a}^{2}, n=1, \ldots, N$, are computed as

$$
\eta_{n}^{\mathrm{s}}:=\left\|\mathbf{v}_{n}^{*}-\hat{\mathbf{u}}_{v}\left(t_{n}^{-}\right)\right\|_{m}^{2}+\left\|\boldsymbol{\sigma}_{n}^{*}-\boldsymbol{\sigma}\left(\hat{\mathbf{u}}_{u}\left(t_{n}^{-}\right)\right)\right\|_{\bar{a}}^{2} .
$$


The error indicator $\eta_{n}^{\mathrm{s}}$ corresponding to time $t_{n}$ is decomposed into elementary contributions $\eta_{n, k}^{\mathrm{s}}$ associated with element $\Omega_{k}$ of the mesh. The elementary contributions $\eta_{n, k}^{\mathrm{s}}$ are computed restricting the integrals in equation (45) to the elements $\Omega_{k}$. The numerical methodology providing $\mathbf{v}_{n}^{*}$ and $\boldsymbol{\sigma}_{n}^{*}$ is detailed below.

The post-processed stress $\boldsymbol{\sigma}_{n}^{*}$ is usually computed using standard stress recovery techniques originally presented for static problems, see $[3,58,59]$. These techniques allow recovering an enhanced continuous stress field $\boldsymbol{\sigma}_{n}^{*}$, which is obtained projecting each component of the space-discontinuous stress field $\boldsymbol{\sigma}\left(\hat{\mathbf{u}}_{u}\left(t_{n}^{-}\right)\right)$into the continuous finite element functional space used to approximate displacements and velocities, see figure 3 . That is, the enhanced stress $\boldsymbol{\sigma}_{n}^{*}$ is recovered in the space

$$
\mathcal{S}^{H}:=\left\{\boldsymbol{\tau} \in\left[C^{0}(\bar{\Omega})\right]^{d(d+1) / 2}:\left.\boldsymbol{\tau}\right|_{\Omega_{k}} \in\left[\mathbb{P}^{p}\left(\Omega_{k}\right)\right]^{d(d+1) / 2}, k=1, \ldots, N_{\mathrm{el}}\right\},
$$

where $\mathbb{P}^{p}\left(\Omega_{k}\right)$ is the space of polynomials of degree $p$ on the element $\Omega_{k}$. In particular, the stress field $\boldsymbol{\sigma}_{n}^{*} \in \mathcal{S}^{H}$ is sought as a linear combination of nodal shape functions, namely

$$
\boldsymbol{\sigma}_{n}^{*}=\sum_{j=1}^{N_{\text {nod }}} \boldsymbol{\sigma}_{n}^{*}\left(\mathbf{x}_{j}\right) N_{j}(\mathbf{x}),
$$

where $N_{j}(\mathbf{x})$ is the finite element shape function of degree $p$ associated to the node $\mathbf{x}_{j}$, being $N_{\text {nod }}$ the total number of nodes. The output of the recovery procedure are the nodal values of the recovered stress field $\boldsymbol{\sigma}_{n}^{*}\left(\mathbf{x}_{j}\right), j=1, \ldots, N_{\text {nod }}$, describing the stress $\boldsymbol{\sigma}_{n}^{*} \in \mathcal{S}^{H}$
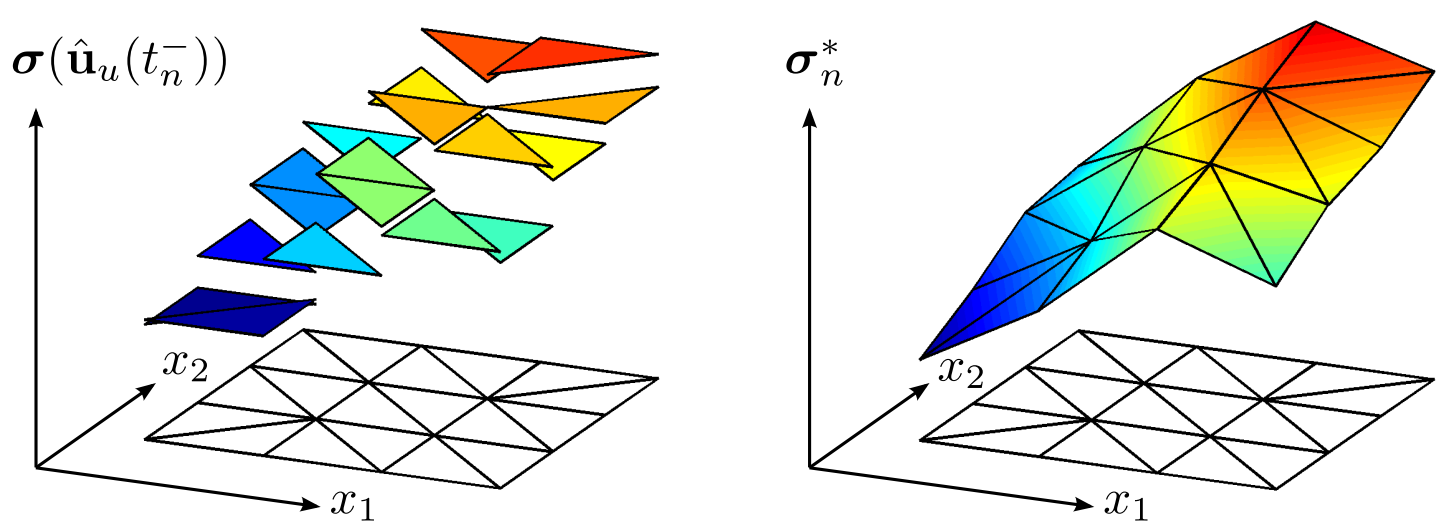

Figure 3: The the discontinuous stress field $\boldsymbol{\sigma}\left(\hat{\mathbf{u}}_{u}\left(t_{n}^{-}\right)\right)$(left) is projected into the continuous space generated by the shape functions furnishing the stress field $\boldsymbol{\sigma}_{n}^{*}$ (right) for all time steps $n=1, \ldots, N$ (illustrated for $p=1$ ).

Several strategies are studied in the literature for the stress recovery. Two of the most common techniques are summarized hereafter. First, the pioneering reference by Zienkiewicz and Zhu [3] proposes to recover the stress field $\boldsymbol{\sigma}_{n}^{*}$ using a global $L^{2}$ projection. Specifically, the recovered stress $\boldsymbol{\sigma}_{n}^{*}$ is found solving the global discrete problem: find $\boldsymbol{\sigma}_{n}^{*} \in \mathcal{S}^{H}$ such that

$$
\left(\boldsymbol{\sigma}_{n}^{*}, \boldsymbol{\tau}\right)=\left(\boldsymbol{\sigma}\left(\hat{\mathbf{u}}_{u}\left(t_{n}^{-}\right)\right), \boldsymbol{\tau}\right) \quad \forall \boldsymbol{\tau} \in \mathcal{S}^{H},
$$


where $(\cdot, \cdot)$ denotes the $L^{2}$-product in $\mathcal{S}^{H}$. Problem (46) is equivalent to a linear system of equations with a global mass matrix, for each component of the stress tensor, and at each time step $t_{n}$. If the space mesh changes, then the mass matrix has to be reassembled and inverted at each time step. Hence, this technique is not very well suited in dynamic problems. In that case, stress recovery techniques involving only local problems are preferred, as the well known SPR technique also introduced by Zienkiewicz and Zhu $[58,59]$.

The SPR technique allows recovering the nodal values of the recovered stress field $\boldsymbol{\sigma}_{n}^{*}\left(\mathbf{x}_{j}\right)$ solving local small problems associated to the mesh nodes (nodal patches). The values $\boldsymbol{\sigma}_{n}^{*}\left(\mathbf{x}_{j}\right)$ are found using the information provided by the set of Gauss points $\boldsymbol{\mathcal { X }}^{\omega_{j}}$ inside the patch $\omega_{j}:=\operatorname{supp}\left(N_{j}\right) \subset \Omega$ surrounding the $j$-th node, see figure 4 . A local stress field

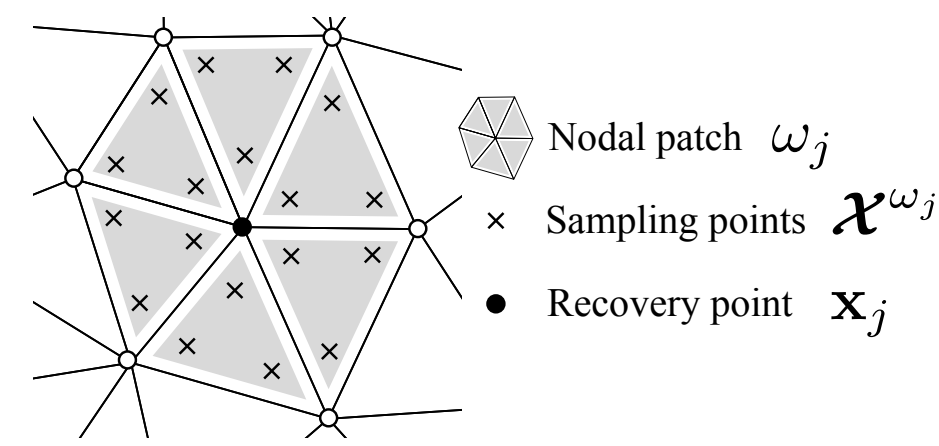

Figure 4: Definition of nodal patches and sampling points for stress recovery.

of polynomial degree $\tilde{p}, \boldsymbol{\sigma}_{n}^{\omega_{j}} \in\left[\mathbb{P}^{\tilde{p}}\left(\omega_{j}\right)\right]^{d(d+1) / 2}$, is defined on the patch $\omega_{j}$. The recovered stresses $\boldsymbol{\sigma}_{n}^{\omega_{j}}$ are computed as the least squares fitting of the values of the stress $\boldsymbol{\sigma}\left(\hat{\mathbf{u}}_{u}\left(t_{n}^{-}\right)\right)$ at the sampling points $\boldsymbol{\mathcal { X }}^{\omega_{j}}$. Specifically, the stress $\boldsymbol{\sigma}_{n}^{\omega_{j}}$ is defined as

$$
\boldsymbol{\sigma}_{n}^{\omega_{j}}:=\arg \min _{\mathbf{w} \in\left[\mathbb{P}^{\tilde{p}}\left(\omega_{j}\right)\right]^{d(d+1) / 2}} \sum_{\mathbf{x} \in \mathcal{X}^{\omega_{j}}}\left(\mathbf{w}(\mathbf{x})-\boldsymbol{\sigma}\left(\hat{\mathbf{u}}_{u}\left(t_{n}^{-}\right)\right)(\mathbf{x})\right)^{2} .
$$

The recovered value $\boldsymbol{\sigma}_{n}^{*}\left(\mathbf{x}_{j}\right)$ is then taken to be the value of the local stress field $\boldsymbol{\sigma}_{n}^{\omega_{j}}$ at point $\mathbf{x}_{j}$, that is $\boldsymbol{\sigma}_{n}^{*}\left(\mathbf{x}_{j}\right):=\boldsymbol{\sigma}_{n}^{\omega_{j}}\left(\mathbf{x}_{j}\right)$. In practice, for linear elements, $\tilde{p}$ is taken equal to 2. In any case, $\tilde{p}$ must be such that the dimension of $\mathbb{P}^{\tilde{p}}\left(\omega_{j}\right)$ is lower then the cardinal of $\mathcal{X}^{\omega_{j}}$ to guarantee that the least squares projection is well posed.

On the other hand, the technique furnishing the enhanced velocities $\mathbf{v}_{n}^{*}$ consists, basically, in increasing the interpolation degree of the computed velocity $\hat{\mathbf{u}}_{v}\left(t_{n}^{-}\right)$, see figure 5 . This type of post-process is used in other contexts, for instance, in assessing the error $L^{2}$-norm in static problems $[60,61]$ or, in building enhanced vibration modes and eigenfrequencies [62]. In the following, the post-process strategy introduced in [62] is presented.

Let $\Omega_{k}^{\text {patch }}$ denote the patch of elements around $\Omega_{k}$, consisting of all the elements sharing at least one node with $\Omega_{k}$, and let $\boldsymbol{\mathcal { X }}_{k}$ and $\boldsymbol{\mathcal { X }}_{k}^{\text {patch }}$ denote the set of nodes of element $\Omega_{k}$ and patch $\Omega_{k}^{\text {patch }}$ respectively, see figure 6 .

The post-processed velocity field $\mathbf{v}_{n}^{*}$ is found using the information provided by the restriction of $\hat{\mathbf{u}}_{v}\left(t_{n}^{-}\right)$to $\Omega_{k}^{\text {patch }}$. Specifically, a velocity field $\mathbf{v}_{n}^{\boldsymbol{X}_{k}} \in\left[\mathbb{P}^{p+1}\left(\Omega_{k}^{\text {patch }}\right)\right]^{d}$ is found 

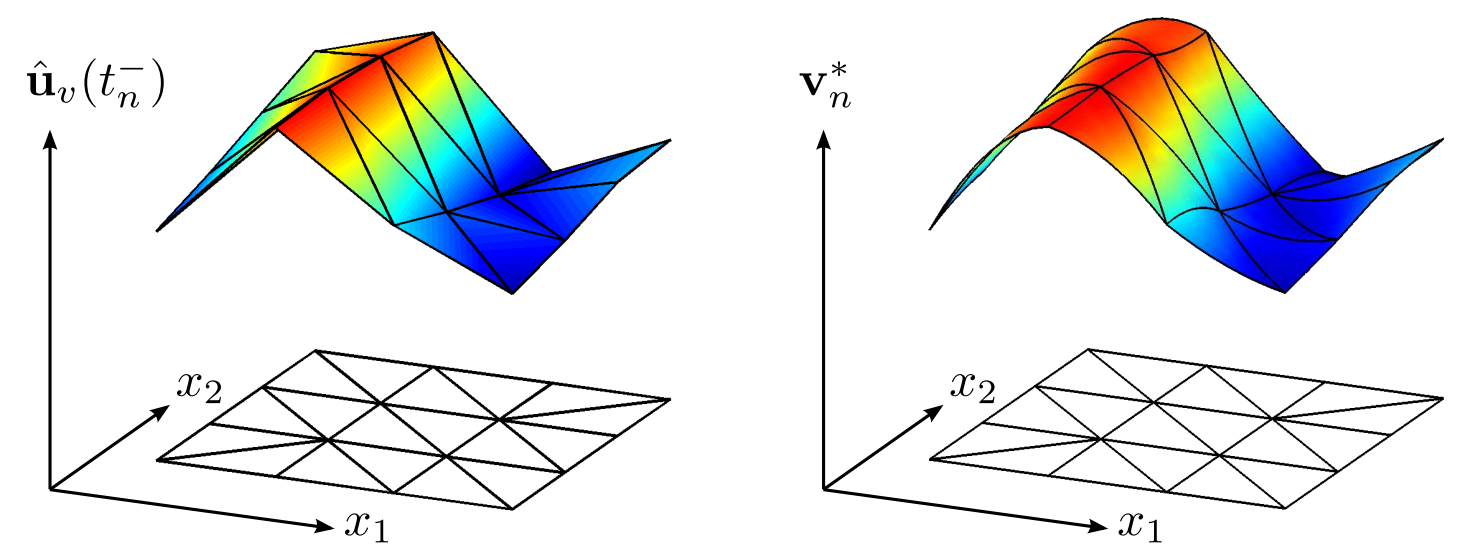

Figure 5: The recovery techniques for the velocities consist in increasing the interpolation order of $\hat{\mathbf{u}}_{v}\left(t_{n}^{-}\right)$(left) furnishing the recovered velocities $\mathbf{v}_{n}^{*}$ (right).
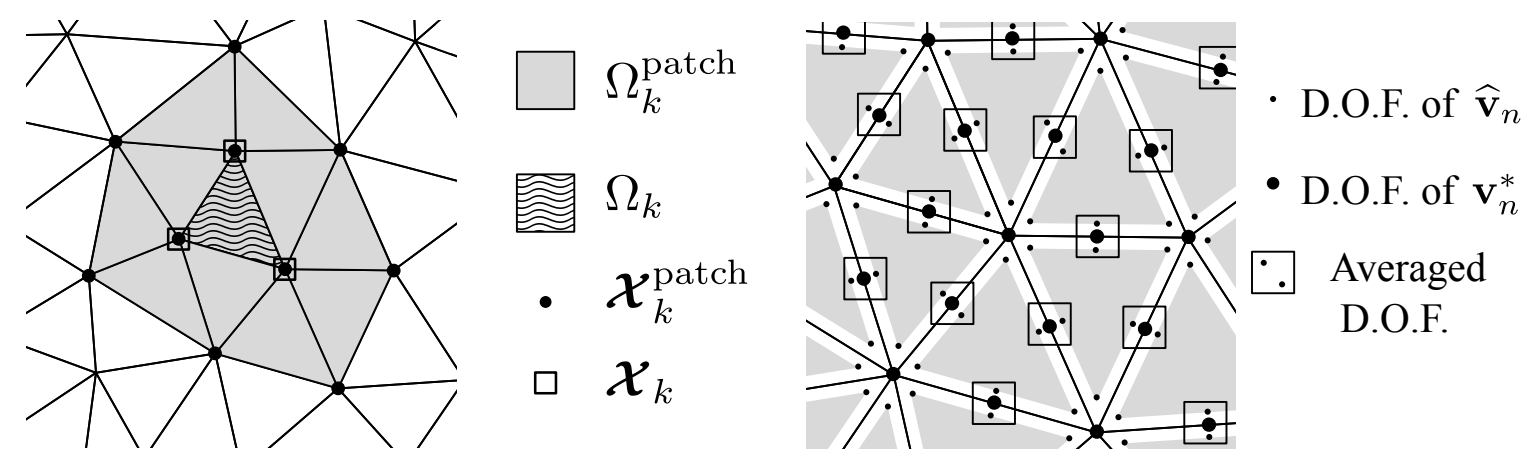

Figure 6: Definition of element patches (left) and illustration of the averaging of discontinuous function $\widehat{\mathbf{v}}_{n}$ into the continuous function $\mathbf{v}_{n}^{*}$ (right).

such that it fits the values of $\hat{\mathbf{u}}_{v}\left(t_{n}^{-}\right)$at $\boldsymbol{\mathcal { X }}_{k}^{\text {patch }}$ in a least squares sense and coincides with $\hat{\mathbf{u}}_{v}\left(t_{n}^{-}\right)$at $\boldsymbol{\mathcal { X }}_{k}$. That is,

$$
\begin{aligned}
\mathbf{v}_{n}^{\mathcal{X}_{k}}=\arg \min _{\mathbf{w} \in\left[\mathbb{P}^{p+1}\left(\Omega_{k}^{\text {patch }}\right)\right]^{d}} & \sum_{\mathbf{x} \in \mathcal{X}_{k}^{\text {patch }}}\left(\mathbf{w}(\mathbf{x})-\hat{\mathbf{u}}_{v}\left(\mathbf{x}, t_{n}^{-}\right)\right)^{2} \\
\text { constrained to } & \mathbf{w}(\mathbf{x})=\hat{\mathbf{u}}_{v}\left(\mathbf{x}, t_{n}^{-}\right) \text {for } \mathbf{x} \in \mathcal{X}_{k} .
\end{aligned}
$$

Problem (48) results in $d$ (one for each component of the velocity, all with the same mass matrix) linear system of equations of size equal to the dimension of $\mathbb{P}^{p+1}$ for each element of the computational mesh.

In order to obtain the post-processed velocity field $\mathbf{v}_{n}^{*}$, first the contributions of the restriction of the local recovered functions $\mathbf{v}_{n}^{\mathcal{X}_{k}}$ to the corresponding element $\Omega_{k}$ are summed, $\widehat{\mathbf{v}}_{n}:=\left.\sum_{k} \mathbf{v}_{n}^{\mathcal{X}_{k}}\right|_{\Omega_{k}}$. Note that $\widehat{\mathbf{v}}_{n}$ is discontinuous because, for two neighboring elements $\Omega_{k}$ and $\Omega_{k^{\prime}}$ with a common side $\Gamma_{k k^{\prime}}:=\bar{\Omega}_{k} \cap \bar{\Omega}_{k^{\prime}}$, functions $\left.\mathbf{v}_{n}^{\mathcal{X}_{k}}\right|_{\Omega_{k}}$ and $\left.\mathbf{v}_{n}^{\mathcal{X}_{k^{\prime}}}\right|_{\Omega_{k^{\prime}}}$ coincide at the endpoints of $\Gamma_{k k^{\prime}}$ but, in general, not in the other points of $\Gamma_{k k^{\prime}}$. In order to build up a continuous approximation $\mathbf{v}_{n}^{*}$, the local contributions are averaged on the 
element sides. Typically $\left.\mathbf{v}_{n}^{\mathcal{X}_{k}}\right|_{\Omega_{k}}$ is represented with the nodal values of a finite element of degree $p+1$. Therefore, computing $\mathbf{v}_{n}^{*}$ is simply performed by just averaging the values of the degrees of freedom associated with the element edges (not vertices), as illustrated in figure 6.

\section{Dual weighted residual \& explicit residual esti- mates}

\subsection{Dual weighted residual method}

This section presents the so-called Dual Weighted Residual (DWR) technique providing estimates for the error in the quantity of interest. This technique is introduced by Rannacher and Stuttmeier in the context of steady state linear elasticity problems [63], but it is also applied to linear elastodynamics [35, 31, 36, 14, 37, 38]. In particular, the dual weighted residual technique is presented here in the context of elastodynamics following [35].

The dual weighted residual methodology provides a scalar estimate $\eta^{\mathrm{dwr}}$ for the error in the quantity of interest

$$
\eta^{\mathrm{dwr}} \approx L_{\mathrm{D}}^{\mathcal{O}}(\mathbf{E}),
$$

where, here, the error $\mathbf{E}$ is defined with respect the solution $\widetilde{\mathbf{U}}$ of the discrete double-field problem (12). Following [35] the developments are restricted to linear time descriptions, that is $q=1$.

The error estimate $\eta^{\mathrm{dwr}}$ is obtained replacing the exact adjoint solution $\mathbf{U}^{\mathrm{d}}$ by an appropriate approximation $\widetilde{\mathbf{U}}^{\mathrm{d}}$ in the error representation (39), namely

$$
\eta^{\mathrm{dwr}}:=R_{\mathrm{CGM}}\left(\widetilde{\mathbf{U}}^{\mathrm{d}}\right) .
$$

The scalar estimate $\eta^{\text {dwr }}$ provides a single scalar quantity, which may be used in the framework of an adaptive procedure as a stopping criterion. That is, to check whether the computed numerical approximation has reached the desired accuracy. Additionally, the dual weighted residual method provides local error indicators (typically element by element) to drive goal-oriented adaptive procedures. This information is used to improve the space-time discretization in order to reduce the error $\eta^{\mathrm{dwr}}$.

Deriving the local error indicators requires rewriting the error representation (41) in such a way that the contributions of the space and time discretization errors are separated. This allows to decide whether the space or the time discretizations (or both) have to be refined. First, in order to separate the space and time errors in the error representation (41), the projection $\Pi^{\Delta t} \boldsymbol{\Pi}^{H} \mathbf{U}^{\mathrm{d}}$ of $\mathbf{U}^{\mathrm{d}}$ in the space $\widehat{\mathcal{W}}^{H, \Delta t} \times \widehat{\mathcal{W}}^{H, \Delta t}$ is introduced, where $\Pi^{\Delta t}$ is a projection of a time dependent function into the space of time piecewise constant functions and $\boldsymbol{\Pi}^{H}$ is the classical nodal interpolation projecting space-dependent functions into $\mathcal{V}_{0}^{H}$. In practice, $\Pi^{\Delta t}$ is defined for a time-dependent 
function $\mathbf{w}$ taking the average of $\mathbf{w}$ inside each time interval $I_{n}$

$$
\left.\Pi^{\Delta t} \mathbf{w}\right|_{I_{n}}:=\frac{1}{\operatorname{meas}\left(I_{n}\right)} \int_{I_{n}} \mathbf{w} \mathrm{d} t .
$$

Note that the error in the projection $\mathbf{w}-\Pi^{\Delta t} \mathbf{w}$ is orthogonal to piecewise constant functions in time

$$
\int_{I_{n}}\left(\mathbf{w}-\Pi^{\Delta t} \mathbf{w}\right) \cdot \mathbf{v} \mathrm{d} t=0 \quad \forall \mathbf{v} \in\left[\mathbb{P}^{0}\left(I_{n}\right)\right]^{d} .
$$

Thus, taking $\mathbf{W}^{H, \Delta t}=\boldsymbol{\Pi}^{\Delta t} \boldsymbol{\Pi}^{H} \mathbf{U}^{\mathrm{d}}$ in (41) yields

$$
L_{\mathrm{D}}^{\mathcal{O}}(\mathbf{E})=R_{\mathrm{CGM}}\left(\mathbf{U}^{\mathrm{d}}-\boldsymbol{\Pi}^{\Delta t} \boldsymbol{\Pi}^{H} \mathbf{U}^{\mathrm{d}}\right) .
$$

Remark 4. Figure 7 illustrates the projection operators $\Pi^{H}$ and $\boldsymbol{\Pi}^{\Delta t}$ using the adjoint velocity $\mathbf{u}_{v}^{\mathrm{d}}$ shown in figure 2 associated to the quantity of interest $L_{1}^{\mathcal{O}}(\cdot)$. The exact adjoint velocity $\mathbf{u}_{v}^{\mathrm{d}} \in \mathcal{W}$ is continuous both in space and time. Function $\Pi^{\Delta t} \mathbf{u}_{v}^{\mathrm{d}} \in \widehat{\mathcal{W}}^{\Delta t}$, with

$$
\widehat{\mathcal{W}}^{\Delta t}:=\left\{\mathbf{v} \in\left[L^{2}\left(I ; \mathcal{V}_{0}\right)\right]^{d}:\left.\mathbf{v}\right|_{I_{n}} \in\left[\mathbb{P}^{q-1}\left(I_{n} ; \mathcal{V}_{0}\right)\right]^{d}, n=1, \ldots, N\right\}
$$

is piecewise polynomial (constant for $q=1$ ) in time. However, the spacial description of functions in $\widehat{\mathcal{W}}^{\Delta t}$ is infinite dimensional. Finally, the fully discrete projection $\Pi^{\Delta t} \boldsymbol{\Pi}^{H} \mathbf{u}_{v}^{\mathrm{d}} \in \widehat{\mathcal{W}}^{H, \Delta t}$ is continuous and piecewise polynomial in space, see figure $\%$.
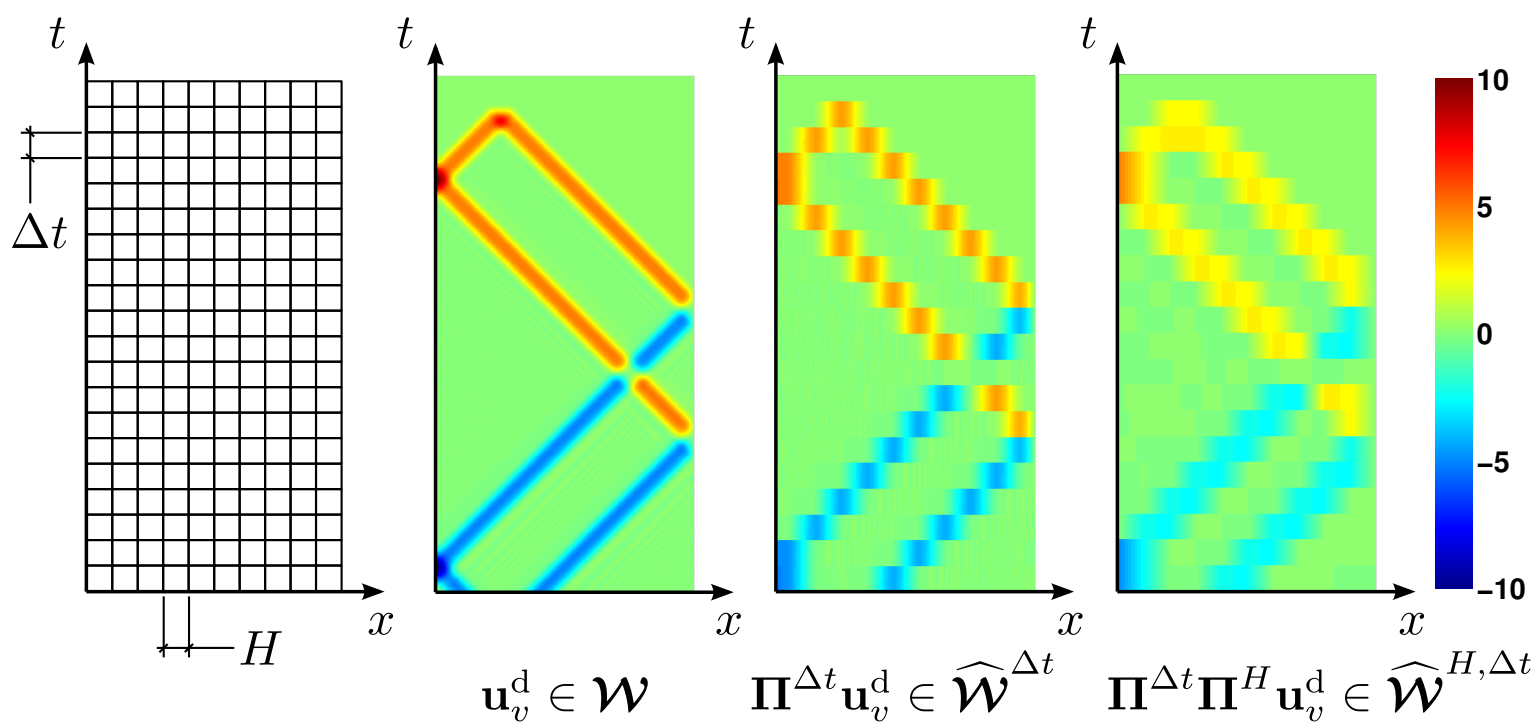

Figure 7: Space-time discretization defining the spaces $\widehat{\mathcal{W}}^{\Delta t}$ and $\widehat{\mathcal{W}}^{H, \Delta t}$ (outer left). Adjoint velocity field $\mathbf{u}_{v}^{\mathrm{d}}$ associated to the quantity of interest $L_{1}^{\mathcal{O}}(\cdot)$ (see figure 2) and its projections $\Pi^{\Delta t} \mathbf{u}_{v}^{\mathrm{d}} \in \widehat{\mathcal{W}}^{\Delta t}$ and $\Pi^{\Delta t} \boldsymbol{\Pi}^{H} \mathbf{u}_{v}^{\mathrm{d}} \in \widehat{\mathcal{W}}^{H, \Delta t}$ (right).

The space and time errors are separated adding and subtracting $\boldsymbol{\Pi}^{\Delta t} \mathbf{U}^{\mathrm{d}}$ in equation (51)

$$
L_{\mathrm{D}}^{\mathcal{O}}(\mathbf{E})=R_{\mathrm{CGM}}\left(\mathbf{U}^{\mathrm{d}}-\boldsymbol{\Pi}^{\Delta t} \mathbf{U}^{\mathrm{d}}\right)+R_{\mathrm{CGM}}\left(\boldsymbol{\Pi}^{\Delta t}\left(\mathbf{U}^{\mathrm{d}}-\boldsymbol{\Pi}^{H} \mathbf{U}^{\mathrm{d}}\right)\right) .
$$


The terms $R_{\mathrm{CGM}}\left(\mathbf{U}^{\mathrm{d}}-\boldsymbol{\Pi}^{\Delta t} \mathbf{U}^{\mathrm{d}}\right)$ and $R_{\mathrm{CGM}}\left(\boldsymbol{\Pi}^{\Delta t}\left(\mathbf{U}^{\mathrm{d}}-\boldsymbol{\Pi}^{H} \mathbf{U}^{\mathrm{d}}\right)\right)$ are associated with the time and space discretization errors respectively. Indeed, $R_{\mathrm{CGM}}\left(\mathbf{U}^{\mathrm{d}}-\boldsymbol{\Pi}^{\Delta t} \mathbf{U}^{\mathrm{d}}\right)$ tends to zero as the time discretization is refined whereas $R_{\mathrm{CGM}}\left(\boldsymbol{\Pi}^{\Delta t}\left(\mathbf{U}^{\mathrm{d}}-\mathbf{\Pi}^{H} \mathbf{U}^{\mathrm{d}}\right)\right)$ tends to zero as the space discretization is refined.

Once the space and time errors are separated, the next step to obtain the local error contributions is splitting the integrals in $R_{\mathrm{CGM}}(\cdot)$ using the space-time cells $\Omega_{k} \times I_{n}$ associated with the elements $\Omega_{k}$ and time intervals $I_{n}$. That is

$$
\begin{aligned}
R_{\mathrm{CGM}}(\mathbf{W}) & =\sum_{n=1}^{N} \sum_{k=1}^{N_{\mathrm{el}}} \int_{I_{n}}\left[\left(\mathbf{f}, \mathbf{w}_{v}\right)_{\Omega_{k}}+(\mathbf{g}, \mathbf{w})_{\Gamma_{\mathrm{N}} \cap \partial \Omega_{k}}-m\left(\dot{\tilde{\mathbf{u}}}_{v}, \mathbf{w}_{v}\right)_{\Omega_{k}}-a\left(\tilde{\mathbf{u}}_{u}, \mathbf{w}_{v}\right)\right] \mathrm{d} t \\
& \sum_{n=1}^{N} \sum_{k=1}^{N_{\mathrm{el}}} \int_{I_{n}}\left(\mathbf{r}_{u}, \mathbf{w}_{u}\right)_{\Omega_{k}} \mathrm{~d} t+\sum_{k=1}^{N_{\mathrm{el}}}\left[\left(\mathbf{r}_{u}^{0}, \mathbf{w}_{u}(0)\right)_{\Omega_{k}}+\left(\mathbf{r}_{v}^{0}, \mathbf{w}_{v}(0)\right)_{\Omega_{k}}\right],
\end{aligned}
$$

where

$$
\mathbf{r}_{u}:=\rho\left(\dot{\tilde{\mathbf{u}}}_{u}-\tilde{\mathbf{u}}_{v}\right), \quad \mathbf{r}_{u}^{0}:=\rho\left(\mathbf{u}_{0}-\tilde{\mathbf{u}}_{u}(0)\right), \quad \mathbf{r}_{v}^{0}:=\rho\left(\mathbf{v}_{0}-\tilde{\mathbf{u}}_{v}(0)\right) .
$$

The residual $R_{\mathrm{CGM}}(\cdot)$ is written in equation (53) for the particular case of pure elasticity $\left(a_{1}=a_{2}=0\right)$ following reference [35].

An alternative format of the residual is derived integrating by parts the term $a\left(\tilde{\mathbf{u}}_{u}, \mathbf{w}_{v}\right)$ in (53). Thus, the strong residuals associated with the interior of the elements and the element boundaries (edges or faces) are introduced

$\mathbf{r}_{v}^{\mathrm{el}}:=\mathbf{f}-\rho \dot{\tilde{\mathbf{u}}}_{v}+\nabla \cdot \boldsymbol{\sigma}\left(\tilde{\mathbf{u}}_{u}\right)$, in $\Omega_{k}, k=1, \ldots, N^{\mathrm{el}}, \quad$ and $\quad \mathbf{r}_{v}^{\mathrm{fa}}:=\left\{\begin{array}{c}-\frac{1}{2} \llbracket \boldsymbol{\sigma}\left(\tilde{\mathbf{u}}_{u}\right) \cdot \mathbf{n} \rrbracket \text { on } \Gamma^{\mathrm{int}} \\ \mathbf{g}-\boldsymbol{\sigma}\left(\tilde{\mathbf{u}}_{u}\right) \cdot \mathbf{n} \text { on } \Gamma_{\mathrm{N}}\end{array}\right.$

being $\Gamma^{\text {int }}$ the set of interelement boundaries (mesh edges or faces). The jump $\llbracket \boldsymbol{\sigma} \cdot \mathbf{n} \rrbracket$ is defined on a generic element interface $\Gamma_{l}=\partial \Omega_{k} \cap \partial \Omega_{k^{\prime}} \in \Gamma^{\text {int }}$ as $\llbracket \boldsymbol{\sigma} \cdot \mathbf{n} \rrbracket:=\left.\boldsymbol{\sigma}\right|_{\Omega_{k}} \cdot \mathbf{n}_{k}+$ $\left.\boldsymbol{\sigma}\right|_{\Omega_{k^{\prime}}} \cdot \mathbf{n}_{k^{\prime}}$ where $\mathbf{n}_{k}$ and $\mathbf{n}_{k^{\prime}}$ are the outward unit normals to $\partial \Omega_{k}$ and $\partial \Omega_{k^{\prime}}$ respectively. Equation (53) is therefore rewritten as

$$
\begin{aligned}
R_{\mathrm{CGM}}(\mathbf{W}) & =\sum_{n=1}^{N} \sum_{k=1}^{N_{\mathrm{el}}} \int_{I_{n}}\left[\left(\mathbf{r}_{u}, \mathbf{w}_{u}\right)_{\Omega_{k}}+\left(\mathbf{r}_{v}^{\mathrm{el}}, \mathbf{w}_{v}\right)_{\Omega_{k}}+\left(\mathbf{r}_{v}^{\mathrm{fa}}, \mathbf{w}_{v}\right)_{\partial \Omega_{k}}\right] \\
& +\sum_{k=1}^{N_{\mathrm{el}}}\left[\left(\mathbf{r}_{u}^{0}, \mathbf{w}_{u}(0)\right)_{\Omega_{k}}+\left(\mathbf{r}_{v}^{0}, \mathbf{w}_{v}(0)\right)_{\Omega_{k}}\right]
\end{aligned}
$$

where functions $\mathbf{r}_{u}, \mathbf{r}_{v}^{\mathrm{el}}, \mathbf{r}_{v}^{\mathrm{fa}}, \mathbf{r}_{u}^{0}$ and $\mathbf{r}_{v}^{0}$ are the computable strong residuals contributing to $R_{\mathrm{CGM}}(\cdot)$. This new format of the residual is interesting because, when restricted to elements and time slabs, it provides better space-time local indicators than the original one (53).

The local (element by element) error contributions associated with the space and time discretization errors are obtained using the residual decomposition (54) in the error 
representation (52):

$$
\begin{aligned}
L_{\mathrm{D}}^{\mathcal{O}}(\mathbf{E}) & =\sum_{k=1}^{N_{\mathrm{el}}}\left[\left(\mathbf{r}_{u}^{0}, \mathbf{u}_{u}^{\mathrm{d}}(0)-\boldsymbol{\Pi}^{\Delta t} \boldsymbol{\Pi}^{H} \mathbf{u}_{u}^{\mathrm{d}}(0)\right)_{\Omega_{k}}+\left(\mathbf{r}_{v}^{0}, \mathbf{u}_{v}^{\mathrm{d}}(0)-\boldsymbol{\Pi}^{\Delta t} \boldsymbol{\Pi}^{H} \mathbf{u}_{v}^{\mathrm{d}}(0)\right]\right. \\
& +\sum_{n=1}^{N} \sum_{k=1}^{N_{\mathrm{el}}} \int_{I_{n}}\left[\left(\mathbf{r}_{u}-\boldsymbol{\Pi}^{\Delta t} \mathbf{r}_{u}, \mathbf{u}_{u}^{\mathrm{d}}-\boldsymbol{\Pi}^{\Delta t} \mathbf{u}_{u}^{\mathrm{d}}\right)_{\Omega_{k}}+\left(\mathbf{r}_{u}, \boldsymbol{\Pi}^{\Delta t} \mathbf{u}_{u}^{\mathrm{d}}-\boldsymbol{\Pi}^{\Delta t} \boldsymbol{\Pi}^{H} \mathbf{u}_{u}^{\mathrm{d}}\right)_{\Omega_{k}}\right] \\
& +\sum_{n=1}^{N} \sum_{k=1}^{N_{\mathrm{el}}} \int_{I_{n}}\left[\left(\mathbf{r}_{v}^{\mathrm{el}}-\boldsymbol{\Pi}^{\Delta t} \mathbf{r}_{v}^{\mathrm{el}}, \mathbf{u}_{v}^{\mathrm{d}}-\boldsymbol{\Pi}^{\Delta t} \mathbf{u}_{v}^{\mathrm{d}}\right)_{\Omega_{k}}+\left(\mathbf{r}_{v}^{\mathrm{el}}, \boldsymbol{\Pi}^{\Delta t} \mathbf{u}_{v}^{\mathrm{d}}-\boldsymbol{\Pi}^{\Delta t} \boldsymbol{\Pi}^{H} \mathbf{u}_{v}^{\mathrm{d}}\right)_{\Omega_{k}}\right] \\
& +\sum_{n=1}^{N} \sum_{k=1}^{N_{\mathrm{el}}} \int_{I_{n}}\left[\left(\mathbf{r}_{v}^{\mathrm{fa}}-\boldsymbol{\Pi}^{\Delta t} \mathbf{r}_{v}^{\mathrm{fa}}, \mathbf{u}_{v}^{\mathrm{d}}-\boldsymbol{\Pi}^{\Delta t} \mathbf{u}_{v}^{\mathrm{d}}\right)_{\partial \Omega_{k}}+\left(\mathbf{r}_{v}^{\mathrm{fa}}, \boldsymbol{\Pi}^{\Delta t} \mathbf{u}_{v}^{\mathrm{d}}-\boldsymbol{\Pi}^{\Delta t} \boldsymbol{\Pi}^{H} \mathbf{u}_{v}^{\mathrm{d}}\right)_{\partial \Omega_{k}}\right],
\end{aligned}
$$

where functions $\Pi^{\Delta t} \mathbf{r}_{u}, \Pi^{\Delta t} \mathbf{r}_{v}^{\mathrm{el}}$ and $\Pi^{\Delta t} \mathbf{r}_{v}^{\mathrm{fa}}$ are introduced into equation (55) using the orthogonality property (50).

Equation (55) leads to local error contributions which might have opposite signs from element to element. In practice, the remeshing criteria, which translate the local error contributions into a desired element size, require that the input local indicators are positive. The positive error contributions are obtained using the Cauchy-Schwartz inequality in equation (55), namely

$$
\left|L_{\mathrm{D}}^{\mathcal{O}}(\mathbf{E})\right| \leq \sum_{k=1}^{N_{\mathrm{el}}} \eta_{k}^{\mathrm{dwr}, \mathrm{i}}+\sum_{n=0}^{N} \sum_{k=1}^{N_{\mathrm{el}}}\left[\eta_{n, k}^{\mathrm{dwr}, \mathrm{s}}+\eta_{n, k}^{\mathrm{dwr}, \mathrm{t}}\right]
$$

with

$$
\begin{aligned}
\eta_{k}^{\mathrm{dwr}, \mathrm{i}} & :=\left\|\mathbf{r}_{u}^{0}\right\|_{\Omega_{k}}\left\|\mathbf{u}_{u}^{\mathrm{d}}(0)-\boldsymbol{\Pi}^{\Delta t} \boldsymbol{\Pi}^{H} \mathbf{u}_{u}^{\mathrm{d}}(0)\right\|_{\Omega_{k}}+\left\|\mathbf{r}_{v}^{0}\right\|_{\Omega_{k}}\left\|\mathbf{u}_{v}^{\mathrm{d}}(0)-\boldsymbol{\Pi}^{\Delta t} \boldsymbol{\Pi}^{H} \mathbf{u}_{v}^{\mathrm{d}}(0)\right\|_{\Omega_{k}}, \\
\eta_{n, k}^{\mathrm{dwr}, \mathrm{t}} & :=\left\|\mathbf{r}_{u}-\boldsymbol{\Pi}^{\Delta t} \mathbf{r}_{u}\right\|_{\Omega_{k} \times I_{n}}\left\|\mathbf{u}_{u}^{\mathrm{d}}-\boldsymbol{\Pi}^{\Delta t} \mathbf{u}_{u}^{\mathrm{d}}\right\|_{\Omega_{k} \times I_{n}}+\left\|\mathbf{r}_{v}^{\mathrm{el}}-\boldsymbol{\Pi}^{\Delta t} \mathbf{r}_{v}^{\mathrm{el}}\right\|_{\Omega_{k} \times I_{n}}\left\|\mathbf{u}_{v}^{\mathrm{d}}-\boldsymbol{\Pi}^{\Delta t} \mathbf{u}_{v}^{\mathrm{d}}\right\|_{\Omega_{k} \times I_{n}} \\
& +\left\|\mathbf{r}_{v}^{\mathrm{fa}}-\boldsymbol{\Pi}^{\Delta t} \mathbf{r}_{v}^{\mathrm{fa}}\right\|_{\partial \Omega_{k} \times I_{n}}\left\|\mathbf{u}_{v}^{\mathrm{d}}-\boldsymbol{\Pi}^{\Delta t} \mathbf{u}_{v}^{\mathrm{d}}\right\|_{\partial \Omega_{k} \times I_{n}}, \\
\eta_{n, k}^{\mathrm{dwr}, \mathrm{s}} & :=\left\|\mathbf{r}_{u}\right\|_{\Omega_{k}}\left\|\boldsymbol{\Pi}^{\Delta t} \mathbf{u}_{u}^{\mathrm{d}}-\boldsymbol{\Pi}^{\Delta t} \boldsymbol{\Pi}^{H} \mathbf{u}_{u}^{\mathrm{d}}\right\|_{\Omega_{k} \times I_{n}}+\left\|\mathbf{r}_{v}^{\mathrm{el}}\right\|_{\Omega_{k} \times I_{n}}\left\|\boldsymbol{\Pi}^{\Delta t} \mathbf{u}_{v}^{\mathrm{d}}-\boldsymbol{\Pi}^{\Delta t} \boldsymbol{\Pi}^{H} \mathbf{u}_{v}^{\mathrm{d}}\right\|_{\Omega_{k} \times I_{n}} \\
& +\left\|\mathbf{r}_{v}^{\mathrm{fa}}\right\|_{\partial \Omega_{k} \times I_{n}}\left\|\boldsymbol{\Pi}^{\Delta t} \mathbf{u}_{v}^{\mathrm{d}}-\boldsymbol{\Pi}^{\Delta t} \boldsymbol{\Pi}^{H} \mathbf{u}_{v}^{\mathrm{d}}\right\|_{\partial \Omega_{k} \times I_{n}},
\end{aligned}
$$

where the notation $\|\cdot\|_{\Omega_{k}},\|\cdot\|_{\Omega_{k} \times I_{n}}$ and $\|\cdot\|_{\partial \Omega_{k} \times I_{n}}$ is used to denote the $L^{2}$ norms in $\Omega_{k}$, $\Omega_{k} \times I_{n}$ and $\partial \Omega_{k} \times I_{n}$ respectively. Note that 1) $\eta_{k}^{\mathrm{dwr}, \mathrm{i}}$ is the contribution of element $\Omega_{k}$ to the interpolation error due to the initial conditions, 2) $\eta_{n, k}^{\mathrm{dwr}, \mathrm{t}}$ is the contribution of the space-time slab $\Omega_{k} \times I_{n}$ to the time discretization error and 3) $\eta_{n, k}^{\mathrm{dwr}, \mathrm{s}}$ is the contribution of the space-time slab $\Omega_{k} \times I_{n}$ to the space discretization error.

The inequality in equation (56) guarantees that the error in the quantity of interest is controlled if the local errors $\eta_{k}^{\mathrm{dwr}, \mathrm{i}}, \eta_{n, k}^{\mathrm{dwr}, \mathrm{s}}, \eta_{n, k}^{\mathrm{dwr}, \mathrm{t}}$ are small enough. Thus, the local error indicators $\eta_{k}^{\mathrm{dwr}, \mathrm{i}}, \eta_{n, k}^{\mathrm{dwr}, \mathrm{s}}, \eta_{n, k}^{\mathrm{dwr}, \mathrm{t}}$ are useful to drive goal-oriented adaptive procedures aiming 
at efficiently controlling $\left|L^{\mathcal{O}}(\mathbf{e})\right|$. However, these local error indicators cannot be used to obtain a reliable assessment of $L^{\mathcal{O}}(\mathbf{e})$, which is better estimated directly with equation (49).

Note that the local error contributions in equation (57) are not fully computable. They involve the norms of the strong residuals which are computable, but they also involve the unknown exact solution of the adjoint problem, $\mathbf{U}^{\mathrm{d}}$. Computable local error indicators from equation (57) are obtained following two alternative approaches.

On the one hand, the exact adjoint solution $\mathbf{U}^{\mathrm{d}}$ is replaced in (57) by a suitable approximation $\widetilde{\mathbf{U}}^{\mathrm{d}}$ as previously done in equation (49). The approximation $\widetilde{\mathbf{U}}^{\mathrm{d}}$ must belong to a richer space than $\widehat{\mathcal{W}}^{H, \Delta t} \times \widehat{\mathcal{W}}^{H, \Delta t}$ in order to preclude Galerkin cancellation. Function $\widetilde{\mathbf{U}}^{\mathrm{d}}$ is computed in $[64,35]$ as post-process of the numerical adjoint approximation using recovery techniques. Alternatively, references [35, 47] compute $\widetilde{\mathbf{U}}^{\mathrm{d}}$ solving a global problem in a richer space obtained with $H$ - or $p$-refinement. This second approach might be computationally unaffordable in three-dimensional demanding problems.

On the other hand, computable local indicators are obtained introducing a priori error estimates for the adjoint interpolation errors $\mathbf{U}^{\mathrm{d}}-\boldsymbol{\Pi}^{\Delta t} \boldsymbol{\Pi}^{H} \mathbf{U}^{\mathrm{d}}$ and $\mathbf{U}^{\mathrm{d}}-\boldsymbol{\Pi}^{\Delta t} \mathbf{U}^{\mathrm{d}}$ appearing in equation (57). This allows to write the adjoint interpolation error in terms of higher order derivatives (both in space and time) of $\mathbf{U}^{\mathrm{d}}$. Then, the unknown high order derivatives of $\mathbf{U}^{\mathrm{d}}$ are replaced by a post-process of the computed adjoint approximation, see $[31,35]$ for details. The use of a priori interpolation estimates introduces unknown constants in the final expression of the estimate. However, the local information given by the computable part of the estimate is used to perform space-time mesh adaptivity.

\subsection{An $L^{2}$-norm explicit estimate}

This section briefly summarizes reference [50] deriving explicit estimates for the $L^{2}$-norm of the final displacement error, $\left\|\mathbf{e}_{u}(T)\right\|=\left(\mathbf{e}_{u}(T), \mathbf{e}_{u}(T)\right)^{1 / 2}$. An analogous rationale holds for assessing the total energy of the error, $\left\|\mathbf{e}_{v}(T)\right\|_{m}^{2}+\left\|\mathbf{e}_{u}(T)\right\|_{a}^{2}$, see references $[34,53]$.

Here, the $L^{2}$-norm of the error is seen as a particular quantity of interest to be estimated using the DWR approach presented in section 5.1. The non-linear character of this quantity of interest induces a corresponding functional output $L_{\mathrm{D}}^{\mathcal{O}}(\cdot)$ involving the unknown error $\mathbf{e}_{u}(T)$. This functional output induces an adjoint problem that plays a role in the derivation of the estimate. However, it is worth mentioning that the estimate is explicit and it does not require solving any adjoint problem.

The presentation in reference [50] considers the wave equation as model problem (with a scalar unknown). The equations of structural dynamics (1) are a general framework (with vectorial unknown) for a second order hyperbolic problem, that are seen as a generalization of the wave equation. Thus, the concepts introduced in [50] are presented here in the framework of problem (1) for the sake of a unified exposition.

Following [50], the Dirichlet boundary conditions are defined on the whole boundary, $\Gamma_{\mathrm{D}}=\partial \Omega$, the density is taken $\rho=1$ and the initial conditions are assumed to be exactly represented by the numerical approximation, that is $\mathbf{u}_{0}-\tilde{\mathbf{u}}_{u}(0)=\mathbf{0}$ and $\mathbf{v}_{0}-\tilde{\mathbf{u}}_{v}(0)=\mathbf{0}$. 
The global $L^{2}$-norm of the final displacement error, $\left\|\mathbf{e}_{u}(T)\right\|$, is assessed introducing the auxiliary quantity of interest

$$
L_{\mathrm{D}}^{\mathcal{O}}(\mathbf{W}):=\left(\mathbf{e}_{u}(T), \mathbf{w}_{u}(T)\right) .
$$

Note that, with this definition, the error in the quantity of interest is indeed the $L^{2}$-norm of the final displacement error,

$$
L_{\mathrm{D}}^{\mathcal{O}}(\mathbf{E})=\left(\mathbf{e}_{u}(T), \mathbf{e}_{u}(T)\right)=\left\|\mathbf{e}_{u}(T)\right\|^{2} .
$$

Assuming that $\mathbf{U}^{\mathrm{d}}$ is the solution of the adjoint problem (34) associated with the quantity of interest (58), the value $\left\|\mathbf{e}_{u}(T)\right\|$ can be expressed using the error representation (41). That is

$$
\left\|\mathbf{e}_{u}(T)\right\|^{2}=L_{\mathrm{D}}^{\mathcal{O}}(\mathbf{E})=R_{\mathrm{CGA}}\left(\mathbf{U}^{\mathrm{d}}-\boldsymbol{\Pi}^{\Delta t} \boldsymbol{\Pi}^{H} \mathbf{U}^{\mathrm{d}}\right),
$$

where $\mathbf{W}^{H, \Delta t}$ is replaced in (41) by the projection of the adjoint solution $\Pi^{\Delta t} \boldsymbol{\Pi}^{H} \mathbf{U}^{\mathrm{d}}$. The error representation (59) involves the residual $R_{\mathrm{CGA}}(\cdot)$ instead of $R_{\mathrm{CGM}}(\cdot)$ because the numerical approximation $\widetilde{\mathbf{U}}$ is computed in reference [50] with the discrete CGA problem (14), and consequently, the orthogonality property holds only for $R_{\mathrm{CGA}}(\cdot)$.

The space and time errors are separated adding and subtracting the projection $\Pi^{H} \mathbf{U}^{d}$ into equation (59),

$$
L_{\mathrm{D}}^{\mathcal{O}}(\mathbf{E})=R_{\mathrm{CGA}}\left(\mathbf{U}^{\mathrm{d}}-\boldsymbol{\Pi}^{H} \mathbf{U}^{\mathrm{d}}\right)+R_{\mathrm{CGA}}\left(\boldsymbol{\Pi}^{H}\left(\mathbf{U}^{\mathrm{d}}-\boldsymbol{\Pi}^{\Delta t} \mathbf{U}^{\mathrm{d}}\right)\right),
$$

where the terms $R_{\mathrm{CGA}}\left(\mathbf{U}^{\mathrm{d}}-\boldsymbol{\Pi}^{H} \mathbf{U}^{\mathrm{d}}\right)$ and $R_{\mathrm{CGA}}\left(\boldsymbol{\Pi}^{H}\left(\mathbf{U}^{\mathrm{d}}-\boldsymbol{\Pi}^{\Delta t} \mathbf{U}^{\mathrm{d}}\right)\right)$ are related with the space and time discretization errors respectively. The space projection operator $\boldsymbol{\Pi}^{H}$ is defined here as the $L^{2}$-projection in the space $\mathcal{V}_{0}^{H}$ instead of the usual nodal interpolation, see reference [50]. This technicality is required to ensure some orthogonality properties. That is, $\boldsymbol{\Pi}^{H} \mathbf{w}$ is defined for a generic function $\mathbf{w} \in \mathcal{V}_{0}$ as the solution of the problem: find $\boldsymbol{\Pi}^{H} \mathbf{w} \in \mathcal{V}_{0}^{H}$ such that

$$
\left(\boldsymbol{\Pi}^{H} \mathbf{w}, \mathbf{v}\right)=(\mathbf{w}, \mathbf{v}), \quad \forall \mathbf{v} \in \mathcal{V}_{0}^{H}
$$

The derivation of the explicit error estimate is split into three conceptual steps: 1) equation (60) is rewritten using the orthogonality properties of the operators $\boldsymbol{\Pi}^{H}$ and $\boldsymbol{\Pi}^{\Delta t}$ and integrating by parts in time, 2) the adjoint interpolation errors are expressed in terms of high order derivatives of the adjoint solution using a priori error estimates and 3) the resulting high order derivatives are bounded using a stability property of the adjoint solution. These steps are detailed below.

First, using the orthogonality properties of the operators $\Pi^{H}$ and $\Pi^{\Delta t}$, equation (60) is rewritten as

$$
\begin{aligned}
L_{\mathrm{D}}^{\mathcal{O}}(\mathbf{E}) & =\int_{I}\left(\mathbf{f}-\boldsymbol{\Pi}^{H} \mathbf{f}, \dot{\mathbf{u}}^{\mathrm{d}}-\boldsymbol{\Pi}^{H} \dot{\mathbf{u}}^{\mathrm{d}}\right) \mathrm{d} t+\int_{I}\left(\mathbf{f}-\boldsymbol{\Pi}^{\Delta t} \mathbf{f}, \boldsymbol{\Pi}^{H}\left(\dot{\mathbf{u}}^{\mathrm{d}}-\boldsymbol{\Pi}^{\Delta t} \dot{\mathbf{u}}^{\mathrm{d}}\right)\right) \mathrm{d} t \\
& -\int_{I} a\left(\tilde{\mathbf{u}}_{u}, \dot{\mathbf{u}}^{\mathrm{d}}-\boldsymbol{\Pi}^{H} \dot{\mathbf{u}}^{\mathrm{d}}\right) \mathrm{d} t-\int_{I} a\left(\tilde{\mathbf{u}}_{u}-\boldsymbol{\Pi}^{\Delta t} \tilde{\mathbf{u}}_{u}, \boldsymbol{\Pi}^{H}\left(\dot{\mathbf{u}}^{\mathrm{d}}-\boldsymbol{\Pi}^{\Delta t} \dot{\mathbf{u}}^{\mathrm{d}}\right)\right) \mathrm{d} t \\
& -\int_{I} a\left(\dot{\tilde{\mathbf{u}}}_{u}-\tilde{\mathbf{u}}_{v}, \mathbf{u}^{\mathrm{d}}-\boldsymbol{\Pi}^{H} \mathbf{u}^{\mathrm{d}}\right) \mathrm{d} t+\int_{I} a\left(\tilde{\mathbf{u}}_{v}-\boldsymbol{\Pi}^{\Delta t} \tilde{\mathbf{u}}_{v}, \boldsymbol{\Pi}^{H}\left(\mathbf{u}^{\mathrm{d}}-\boldsymbol{\Pi}^{\Delta t} \mathbf{u}^{\mathrm{d}}\right)\right) \mathrm{d} t .
\end{aligned}
$$


Then, integrating by parts the time integrals in the term

$$
\int_{I} a\left(\tilde{\mathbf{u}}_{u}, \dot{\mathbf{u}}^{\mathrm{d}}-\boldsymbol{\Pi}^{H} \dot{\mathbf{u}}^{\mathrm{d}}\right) \mathrm{d} t
$$

equation (61) yields

$$
\begin{aligned}
L_{\mathrm{D}}^{\mathcal{O}}(\mathbf{E}) & =\int_{I}\left(\mathbf{f}-\boldsymbol{\Pi}^{H} \mathbf{f}, \dot{\mathbf{u}}^{\mathrm{d}}-\boldsymbol{\Pi}^{H} \dot{\mathbf{u}}^{\mathrm{d}}\right) \mathrm{d} t+\int_{I}\left(\mathbf{f}-\boldsymbol{\Pi}^{\Delta t} \mathbf{f}, \boldsymbol{\Pi}^{H}\left(\dot{\mathbf{u}}^{\mathrm{d}}-\boldsymbol{\Pi}^{\Delta t} \dot{\mathbf{u}}^{\mathrm{d}}\right)\right) \mathrm{d} t \\
& -\int_{I} a\left(\tilde{\mathbf{u}}_{u}-\boldsymbol{\Pi}^{\Delta t} \tilde{\mathbf{u}}_{u}, \boldsymbol{\Pi}^{H}\left(\dot{\mathbf{u}}^{\mathrm{d}}-\boldsymbol{\Pi}^{\Delta t} \dot{\mathbf{u}}^{\mathrm{d}}\right)\right) \mathrm{d} t \\
& +\int_{I} a\left(\tilde{\mathbf{u}}_{v}, \mathbf{u}^{\mathrm{d}}-\boldsymbol{\Pi}^{H} \mathbf{u}^{\mathrm{d}}\right) \mathrm{d} t+\int_{I} a\left(\tilde{\mathbf{u}}_{v}-\boldsymbol{\Pi}^{\Delta t} \tilde{\mathbf{u}}_{v}, \boldsymbol{\Pi}^{H}\left(\mathbf{u}^{\mathrm{d}}-\boldsymbol{\Pi}^{\Delta t} \mathbf{u}^{\mathrm{d}}\right)\right) \mathrm{d} t \\
& -a\left(\tilde{\mathbf{u}}_{u}(T), \mathbf{u}^{\mathrm{d}}(T)-\boldsymbol{\Pi}^{H} \mathbf{u}^{\mathrm{d}}(T)\right)+a\left(\tilde{\mathbf{u}}_{u}(0), \mathbf{u}^{\mathrm{d}}(0)-\boldsymbol{\Pi}^{H} \mathbf{u}^{\mathrm{d}}(0)\right) .
\end{aligned}
$$

Second, previous equation is rewritten using a priori error estimates for the interpolation errors $\mathbf{u}^{\mathrm{d}}-\boldsymbol{\Pi}^{H} \mathbf{u}^{\mathrm{d}}$ and $\mathbf{u}^{\mathrm{d}}-\boldsymbol{\Pi}^{\Delta t} \mathbf{u}^{\mathrm{d}}$, see reference [50] for details:

$$
\begin{aligned}
\left|L_{\mathrm{D}}^{\mathcal{O}}(\mathbf{E})\right| & \leq C_{1}\left(\max _{t \in I}\left\|\ddot{\mathbf{u}}^{\mathrm{d}}(t)\right\|\right)\left(\int_{I} \Delta t\left\|\mathbf{f}-\boldsymbol{\Pi}^{\Delta t} \mathbf{f}\right\| \mathrm{d} t+\int_{I} \Delta t\left\|\boldsymbol{\Delta}_{H}\left(\tilde{\mathbf{u}}_{u}-\boldsymbol{\Pi}^{\Delta t} \tilde{\mathbf{u}}_{u}\right)\right\| \mathrm{d} t\right) \\
& +C_{2}\left(\max _{t \in I}\left\|\dot{\mathbf{u}}^{\mathrm{d}}(t)\right\|_{a}\right)\left(\int_{I}\left\|H\left(\mathbf{f}-\boldsymbol{\Pi}^{H} \mathbf{f}\right)\right\| \mathrm{d} t+\int_{I} \Delta t\left\|\tilde{\mathbf{u}}_{v}-\boldsymbol{\Pi}^{\Delta t} \tilde{\mathbf{u}}_{v}\right\|_{a} \mathrm{~d} t\right) \\
& +C_{3}\left(\max _{t \in I}\left\|\boldsymbol{\nabla} \cdot \boldsymbol{\sigma}^{\mathrm{d}}\left(\mathbf{u}^{\mathrm{d}}(t)\right)\right\|\right)\left(\int_{I}\left\|H^{2} R_{2}\left(\tilde{\mathbf{u}}_{v}\right)\right\| \mathrm{d} t+\left\|H^{2} R_{2}\left(\tilde{\mathbf{u}}_{u}(T)\right)\right\|+\left\|H^{2} R_{2}\left(\tilde{\mathbf{u}}_{u}(0)\right)\right\|\right),
\end{aligned}
$$

where the discrete laplacian operator $\boldsymbol{\Delta}_{H}$ is defined for a generic function $\mathbf{w} \in \mathcal{V}_{0}$ as : find $\boldsymbol{\Delta}_{H} \mathbf{w} \in \mathcal{V}_{0}^{H}$ such that

$$
\left(\boldsymbol{\Delta}_{H} \mathbf{w}, \mathbf{v}\right)=a(\mathbf{w}, \mathbf{v}), \quad \forall \mathbf{w} \in \mathcal{V}_{0}^{H},
$$

and the operator $R_{2}(\cdot)$ is defined for a generic function $\mathbf{w} \in \mathcal{V}_{0}^{H}$ as

$$
\left.R_{2}(\mathbf{w})\right|_{\Omega_{k}}:=\frac{1}{2 H_{k}} \max _{\mathbf{x} \in \partial \Omega_{k}}\left|\llbracket \mathbf{s}^{\mathrm{E}}(\mathbf{w}(\mathbf{x})) \cdot \mathbf{n}(\mathbf{x}) \rrbracket\right| .
$$

Third, the factors in (63) involving the adjoint solution are bounded in terms of the error $\left\|\mathbf{e}_{u}(T)\right\|$ using the following stability property of the adjoint solution $\mathbf{u}^{\mathrm{d}}$ associated with the quantity of interest (58).

Theorem 1. The solution $\mathbf{u}^{\mathrm{d}}$ of the adjoint problem (34) for quantity defined in (58), which strong form is

$$
\begin{aligned}
& \rho \ddot{\mathbf{u}}^{\mathrm{d}}-\boldsymbol{\nabla} \cdot \boldsymbol{\sigma}^{\mathrm{d}}\left(\mathbf{u}^{\mathrm{d}}\right)=\mathbf{0} \quad \text { in } \Omega \times I, \\
& \mathbf{u}^{\mathrm{d}}=\mathbf{0} \quad \text { on } \Gamma_{D} \times I, \\
& \boldsymbol{\sigma}^{\mathrm{d}} \cdot \mathbf{n}=\mathbf{0} \text { on } \Gamma_{N} \times I, \\
& -\boldsymbol{\nabla} \cdot \boldsymbol{\sigma}\left(\mathbf{u}^{\mathrm{d}}\right)=\mathbf{e}_{u} \quad \text { at } \Omega \times\{T\}, \\
& \dot{\mathbf{u}}^{\mathrm{d}}=\mathbf{0} \quad \text { at } \Omega \times\{T\},
\end{aligned}
$$


fulfills

$$
\max _{t \in I}\left(\left\|\ddot{\mathbf{u}}^{\mathrm{d}}(t)\right\|^{2}+\left\|\dot{\mathbf{u}}^{\mathrm{d}}(t)\right\|_{a}^{2}+\left\|\boldsymbol{\nabla} \cdot \boldsymbol{\sigma}\left(\mathbf{u}^{\mathrm{d}}(t)\right)\right\|^{2}\right) \leq C\left\|\mathbf{e}_{u}(T)\right\|^{2} .
$$

The proof can be found in [50, Lemma 17.3].

Using the stability property (65) and recalling that $L_{\mathrm{D}}^{\mathcal{O}}(\mathbf{E})=\left\|\mathbf{e}_{u}(T)\right\|^{2}$, equation (63) yields

$$
\begin{aligned}
\left\|\mathbf{e}_{u}(T)\right\| \leq C\left(\int_{I} \Delta t\left\|\mathbf{f}-\Pi^{\Delta t} \mathbf{f}\right\| \mathrm{d} t+\int_{I} \Delta t\left\|\boldsymbol{\Delta}_{H}\left(\tilde{\mathbf{u}}_{u}-\Pi^{\Delta t} \tilde{\mathbf{u}}_{u}\right)\right\| \mathrm{d} t\right. \\
\quad+\int_{I} \Delta t\left\|\tilde{\mathbf{u}}_{v}-\boldsymbol{\Pi}^{\Delta t} \tilde{\mathbf{u}}_{v}\right\|_{a} \mathrm{~d} t+\int_{I}\left\|H\left(\mathbf{f}-\Pi^{H} \mathbf{f}\right)\right\| \mathrm{d} t \\
\left.\quad+\int_{I}\left\|H^{2} R_{2}\left(\tilde{\mathbf{u}}_{v}\right)\right\| \mathrm{d} t+\left\|H^{2} R_{2}\left(\tilde{\mathbf{u}}_{u}(T)\right)\right\|+\left\|H^{2} R_{2}\left(\tilde{\mathbf{u}}_{u}(0)\right)\right\|\right) .
\end{aligned}
$$

Constant $C$ in the previous expression is unknown. Nevertheless the computable part of the error estimate is split into space-time local contributions providing information on the relative magnitude of the error generated at each region of the computational domain. This information can be used for adaptive purposes. The three first terms in the right hand side of equation (66) are associated with the time discretization error while the four last terms are associated with the space discretization. The local contributions associated with these terms are used to adapt the corresponding space or time discretization.

\section{Constitutive relation error and implicit estimates}

This section aims at computing bounds for the error in the dissipation norm $\|\mathbf{e}\|$ and in the quantity of interest $L^{\mathcal{O}}(\mathbf{e})$ using the so-called constitutive relation error estimates [9]. These estimates require an underlying stress equilibration technique based on solving local problems. The residual is playing the role of the loading of the local problems, and therefore the solution is not an explicit post-process of the residual. Thus, these strategies are also denoted as implicit residual type estimates. In many contexts, constitutive relation error estimates and implicit residual type estimates are fully equivalent.

The goal is to compute scalar values $\eta_{\text {ener }}, \eta_{\mathrm{L}}$ and $\eta_{\mathrm{U}}$ such that

$$
\|\mathbf{e}\| \leq \eta_{\text {ener }} \text { and } \eta_{\mathrm{L}} \leq L^{\mathcal{O}}(\mathbf{e}) \leq \eta_{\mathrm{U}} .
$$

Deriving the error bounds $\eta_{\text {ener }}, \eta_{\mathrm{L}}$ and $\eta_{\mathrm{U}}$ using constitutive relation estimates requires that problem (1) contains some damping (i.e. either $a_{1}$ or $a_{2}$ is different from zero). This means that the bounding properties of the estimate are lost in the limit case of pure elasticity $\left(a_{1}=a_{2}=0\right)$. Non-zero damping allows computing the error bounds (67) following a rationale analogous to the one used in steady-state problems [9].

The technique providing $\eta_{\text {ener }}, \eta_{\mathrm{L}}$ and $\eta_{\mathrm{U}}$ is presented here following reference [46], where the model problem under consideration corresponds to taking $a_{1}=0$ and $a_{2}>0$ in equations (1). Thus, in the remaining of this section, the coefficient $a_{1}$ is assumed to be 
zero and $a_{2}$ is assumed to be strictly positive. The same rationale holds for other damped versions of problem (1). For instance, references [44, 42, 45] introduce damping in the constitutive relation (2) using the linear Maxwell viscous model.

\subsection{Computable upper bounds for the dissipation norm}

The key ingredient to compute error bounds is building a pair $(\tilde{\boldsymbol{\sigma}}, \tilde{\mathbf{u}})$ of a dynamically admissible (D-admissible) stress and a kinematically admissible (K-admissible) displacement.

On the one hand, the set of K-admissible displacements is defined as

$$
\mathcal{U}:=\left\{\mathbf{w} \in \mathcal{W}: \mathbf{w}=\mathbf{u}_{0} \text { at } \Omega \times\{0\} \text { and } \dot{\mathbf{w}}=\mathbf{v}_{0} \text { at } \Omega \times\{0\}\right\} .
$$

Functions in $\mathcal{U}$ are continuous with continuous time derivative and exactly fulfilling both the homogeneous Dirichlet condition (1b) and the initial conditions (1d) and (1e). On the other hand, the space of D-admissible stresses is defined for a given K-admissible displacement $\tilde{\mathbf{u}} \in \mathcal{U}$ as

$$
\mathcal{S}(\tilde{\mathbf{u}}):=\left\{\boldsymbol{\tau} \in \mathcal{Z}: \int_{I}(\boldsymbol{\tau}, \boldsymbol{\varepsilon}(\dot{\mathbf{w}})) \mathrm{d} t=\int_{I}(l(t ; \dot{\mathbf{w}})-(\rho \ddot{\tilde{\mathbf{u}}}, \dot{\mathbf{w}})) \mathrm{d} t \quad \forall \mathbf{w} \in \mathcal{W}\right\}
$$

where

$$
\mathcal{Z}:=\left\{\boldsymbol{\tau}:[\boldsymbol{\tau}]_{i j} \in L^{2}(\Omega \times I) \quad i, j \leq d\right\},
$$

and for $\boldsymbol{\tau}, \boldsymbol{\varepsilon} \in \mathcal{Z}$, the standard $L^{2}$ product in $\Omega$ reads

$$
(\tau, \varepsilon):=\int_{\Omega} \tau: \varepsilon \mathrm{d} \Omega
$$

The stress tensors in $\mathcal{S}(\tilde{\mathbf{u}})$ are in dynamic equilibrium with respect the external loads plus the inertia forces associated with $\ddot{\tilde{\mathbf{u}}}$. For that reason, the definition of (and the notation for) the set $\mathcal{S}(\tilde{\mathbf{u}})$ depends on the K-admissible displacement $\tilde{\mathbf{u}}$. A stress tensor $\tilde{\boldsymbol{\sigma}} \in \mathcal{S}(\tilde{\mathbf{u}})$ is generally discontinuous between mesh elements, while the traction vector $\tilde{\boldsymbol{\sigma}} \cdot \mathbf{n}$ is continuous across element edges (or faces in $3 \mathrm{D}$ ). The weak form of the dynamic equilibrium is implicitly stated in the definition of $\mathcal{S}(\tilde{\mathbf{u}})$ given in (68). The equivalent strong formulation for D-admissibility enforces point wise equilibrium in the interior of the elements and traction continuity across the element interfaces. Thus, for a given finite element mesh, a D-admissible stress $\tilde{\boldsymbol{\sigma}}$ fulfills

$$
\begin{aligned}
-\nabla \cdot \tilde{\boldsymbol{\sigma}} & =\mathbf{f}-\rho \ddot{\tilde{\mathbf{u}}} \quad \text { on } \Omega^{\text {int }} \times I, \\
\tilde{\boldsymbol{\sigma}} \cdot \mathbf{n} & =\mathbf{g} \quad \text { on } \Gamma_{\mathrm{N}} \times I, \\
\llbracket \tilde{\boldsymbol{\sigma}} \cdot \mathbf{n} \rrbracket & =\mathbf{0} \quad \text { on } \Gamma^{\text {int }} \times I,
\end{aligned}
$$

where $\Omega^{\text {int }}$ is the interior of the elements of the mesh and $\Gamma^{\text {int }}$ is the set of interelement faces (or edges in 2D). 
The admissible pair $(\tilde{\boldsymbol{\sigma}}, \tilde{\mathbf{u}}) \in \mathcal{S}(\tilde{\mathbf{u}}) \times \mathcal{U}$ defines the following stress error

$$
\tilde{\boldsymbol{\sigma}}^{\mathrm{e}}:=\tilde{\boldsymbol{\sigma}}-\boldsymbol{\sigma}(\tilde{\mathbf{u}}),
$$

which corresponds to the non verification of the constitutive relation (2). The so-called constitutive relation error (following the terminology by Ladevèze and co-workers) is then computed as $\left\|\tilde{\boldsymbol{\sigma}}^{\mathrm{e}}\right\|_{\sigma}$ where $\|\cdot\|_{\sigma}$ is the stress version of the space-time norm $\|\cdot\|$ defined in (23), namely

$$
\|\boldsymbol{\tau}\|_{\sigma}^{2}:=\frac{1}{a_{2}} \int_{I}\|\boldsymbol{\tau}\|_{\bar{a}}^{2} \mathrm{~d} t .
$$

For the particular case $a_{1}=0$, the displacement and stress norms are related by $\|\mathbf{w}\|=$ $\left\|a_{2} \mathcal{C}: \varepsilon(\dot{\mathbf{w}})\right\|_{\sigma}$.

The constitutive relation error $\left\|\tilde{\boldsymbol{\sigma}}^{\mathrm{e}}\right\|_{\sigma}$ is computable once the fields $\tilde{\boldsymbol{\sigma}}$ and $\tilde{\mathbf{u}}$ are available. Note that, $\left\|\tilde{\boldsymbol{\sigma}}^{\mathrm{e}}\right\|_{\sigma}=0$ if and only if $\tilde{\boldsymbol{\sigma}}=\boldsymbol{\sigma}$ and $\tilde{\mathbf{u}}=\mathbf{u}$. Consequently, $\left\|\tilde{\boldsymbol{\sigma}}^{\mathrm{e}}\right\|_{\sigma}$ is adopted as a pertinent error measure. Moreover, the value $\left\|\tilde{\boldsymbol{\sigma}}^{\mathrm{e}}\right\|_{\sigma}$ provides information about the unknown error e, as shown by the following theorem. For the sake of simplifying, the operators identifying the elastic and viscous contributions of the constitutive law are introduced as $\mathbf{s}^{\mathrm{E}}(\mathbf{u}):=\mathcal{C}: \varepsilon(\mathbf{u})$ and $\mathbf{s}^{\nu}(\mathbf{u}):=a_{2} \mathcal{C}: \varepsilon(\dot{\mathbf{u}})$.

Theorem 2. Given an admissible pair $(\tilde{\boldsymbol{\sigma}}, \tilde{\mathbf{u}}) \in \mathcal{S}(\tilde{\mathbf{u}}) \times \mathcal{U}$, the errors $\mathbf{e}$ and $\tilde{\boldsymbol{\sigma}}^{\mathrm{e}}$ defined in equations (17) and (70), respectively, fulfill

$$
\left\|\tilde{\boldsymbol{\sigma}}^{\mathrm{e}}\right\|_{\sigma}^{2}=\|\dot{\mathbf{e}}(T)\|_{m}^{2}+\|\mathbf{e}(T)\|_{a}^{2}+\|\mathbf{e}\|^{2}+\left\|\boldsymbol{\sigma}^{\nu}-\tilde{\boldsymbol{\sigma}}^{\nu}\right\|_{\sigma}^{2}
$$

where $\boldsymbol{\sigma}^{\nu}:=\mathbf{s}^{\nu}(\mathbf{u})$ and $\tilde{\boldsymbol{\sigma}}^{\nu}:=\tilde{\boldsymbol{\sigma}}-\mathbf{s}^{\mathrm{E}}(\tilde{\mathbf{u}})$.

For the proof, the reader is referred to [46, Theorem 1].

A direct consequence of theorem 2 is the relation $\left\|\tilde{\boldsymbol{\sigma}}^{\mathrm{e}}\right\|_{\sigma}^{2} \geq\|\dot{\mathbf{e}}(T)\|_{m}^{2}+\|\mathbf{e}(T)\|_{a}^{2}+\|\mathbf{e}\|^{2}$ and, in particular, the following upper bound

$$
\eta_{\text {ener }}:=\left\|\tilde{\boldsymbol{\sigma}}^{\mathrm{e}}\right\|_{\sigma} \geq\|\mathbf{e}\|
$$

Moreover, expression (72) is particularly important because it is used to bound the quantity of interest.

\subsection{Error bounds in the quantity of interest}

Bounds of the error in the quantity of interest $L^{\mathcal{O}}(\mathbf{e})$ are obtained combining admissible pairs for both the original and the adjoint problems, $(\tilde{\mathbf{u}}, \tilde{\boldsymbol{\sigma}})$ and $\left(\tilde{\mathbf{u}}^{\mathrm{d}}, \tilde{\boldsymbol{\sigma}}^{\mathrm{d}}\right)$. The space of adjoint kinematically admissible displacements is defined as

$$
\mathcal{U}^{\mathrm{d}}:=\left\{\mathbf{w} \in \mathcal{W}: \mathbf{w}=\mathbf{u}^{\mathcal{O}} \text { at } \Omega \times\{T\} \text { and } \dot{\mathbf{w}}=\mathbf{v}^{\mathcal{O}} \text { at } \Omega \times\{T\}\right\} .
$$

The space of adjoint dynamically admissible stress fields is defined for a given $\tilde{\mathbf{u}}^{\mathrm{d}} \in \mathcal{U}^{\mathrm{d}}$ as

$$
\mathcal{S}^{\mathrm{d}}\left(\tilde{\mathbf{u}}^{\mathrm{d}}\right):=\left\{\boldsymbol{\tau} \in \mathcal{Z}: \int_{I}(\boldsymbol{\tau}, \boldsymbol{\varepsilon}(\dot{\mathbf{w}})) \mathrm{d} t=\int_{I}\left(l^{\mathcal{O}}(t ; \dot{\mathbf{w}})-\left(\rho \ddot{\tilde{\mathbf{u}}}^{\mathrm{d}}, \dot{\mathbf{w}}\right)\right) \mathrm{d} t \quad \forall \mathbf{w} \in \mathcal{W}\right\},
$$


where $l^{\mathcal{O}}(t ; \mathbf{w}):=\left(\mathbf{f}^{\mathcal{O}}(t), \mathbf{w}\right)+\left(\mathbf{g}^{\mathcal{O}}(t), \mathbf{w}\right)_{\Gamma_{\mathrm{N}}}$.

The admissible pair $\left(\tilde{\mathbf{u}}^{\mathrm{d}}, \tilde{\boldsymbol{\sigma}}^{\mathrm{d}}\right) \in \mathcal{U}^{\mathrm{d}} \times \mathcal{S}^{\mathrm{d}}\left(\tilde{\mathbf{u}}^{\mathrm{d}}\right)$ provides the error in stresses associated with the non verification of the adjoint constitutive relation (27),

$$
\tilde{\boldsymbol{\sigma}}^{\mathrm{d}, \mathrm{e}}:=\tilde{\boldsymbol{\sigma}}^{\mathrm{d}}-\boldsymbol{\sigma}^{\mathrm{d}}\left(\tilde{\mathbf{u}}^{\mathrm{d}}\right) .
$$

The bounds for $L^{\mathcal{O}}(\mathbf{e})$ are computed using the constitutive relation errors $\tilde{\boldsymbol{\sigma}}^{\mathrm{e}}$ and $\tilde{\boldsymbol{\sigma}}^{\mathrm{d}, \mathrm{e}}$, as defined in (70) and (73). Actually, $\tilde{\boldsymbol{\sigma}}^{\mathrm{e}}$ and $\tilde{\boldsymbol{\sigma}}^{\mathrm{d}, \mathrm{e}}$ are used to obtain bounds for $R^{\mathrm{d}}(\mathbf{e})$, which is the non-computable part of the error representation (37). There are three different approaches to derive upper bounds on the basis of the constitutive relation errors, which are described in the following.

\subsubsection{Error bounds based on the Cauchy-Schwarz inequality}

References [42, 43, 44, 45] derive computable bounds using the Cauchy-Schwarz inequality. The bounds for $R^{\mathrm{d}}(\mathbf{e})$ are obtained noting that if $\left(\tilde{\mathbf{u}}^{\mathrm{d}}, \tilde{\boldsymbol{\sigma}}^{\mathrm{d}}\right)$ is an adjoint admissible pair, then its associated error $\tilde{\boldsymbol{\sigma}}^{\mathrm{d}, \mathrm{e}}$ verifies

$$
\bar{B}^{\nu}\left(\tilde{\boldsymbol{\sigma}}^{\mathrm{d}, \mathrm{e}}, \mathbf{s}^{\nu}(\mathbf{w})\right)=R^{\mathrm{d}}(\mathbf{w}) \quad \forall \mathbf{w} \in \mathcal{W},
$$

where $\bar{B}^{\nu}(\cdot, \cdot)$ is the bilinear form

$$
\bar{B}^{\nu}\left(\boldsymbol{\tau}_{1}, \boldsymbol{\tau}_{2}\right):=\frac{1}{a_{2}} \int_{I} \bar{a}\left(\boldsymbol{\tau}_{1}, \boldsymbol{\tau}_{2}\right) \mathrm{d} t
$$

Note that the bilinear form $\bar{B}^{\nu}(\cdot, \cdot)$ induces the stress energy norm $\|\cdot\|_{\sigma}$, that is $\|\boldsymbol{\tau}\|_{\sigma}^{2}:=$ $\bar{B}^{\nu}(\boldsymbol{\tau}, \boldsymbol{\tau})$.

Taking $\mathbf{w}=\mathbf{e}$, equation (74) yields

$$
R^{\mathrm{d}}(\mathbf{e})=\bar{B}^{\nu}\left(\tilde{\boldsymbol{\sigma}}^{\mathrm{d}, \mathrm{e}}, \mathbf{s}^{\nu}(\mathbf{e})\right) .
$$

Being $\bar{B}^{\nu}$ symmetric-positive-definite, the Cauchy-Schwarz inequality holds and yields

$$
\left|R^{\mathrm{d}}(\mathbf{e})\right| \leq\left\|\tilde{\boldsymbol{\sigma}}^{\mathrm{d}, \mathrm{e}}\right\|_{\sigma}\left\|\mathbf{s}^{\nu}(\mathbf{e})\right\|_{\sigma}=\left\|\tilde{\boldsymbol{\sigma}}^{\mathrm{d}, \mathrm{e}}\right\|_{\sigma}\|\mathbf{e}\| .
$$

The factor involving the unknown error e is bounded using the equation (72) leading to the following computable bound for $\left|R^{\mathrm{d}}(\mathbf{e})\right|$,

$$
\left|R^{\mathrm{d}}(\mathbf{e})\right| \leq\left\|\tilde{\boldsymbol{\sigma}}^{\mathrm{d}, \mathrm{e}}\right\|_{\sigma}\left\|\tilde{\boldsymbol{\sigma}}^{\mathrm{e}}\right\|_{\sigma} .
$$

The computable bounds for the error in the quantity of interest are readily obtained using the previous result together with the computable part of the error representation (37). That is,

$$
\zeta_{\mathrm{L}}^{\mathrm{C}-\mathrm{S}} \leq L^{\mathcal{O}}(\mathbf{e}) \leq \zeta_{\mathrm{U}}^{\mathrm{C}-\mathrm{S}}
$$

where

$$
\begin{aligned}
& \zeta_{\mathrm{U}}^{\mathrm{C}-\mathrm{S}}:=\left\|\tilde{\boldsymbol{\sigma}}^{\mathrm{d}, \mathrm{e}}\right\|_{\sigma}\left\|\tilde{\boldsymbol{\sigma}}^{\mathrm{e}}\right\|_{\sigma}+R\left(\tilde{\mathbf{u}}^{\mathrm{d}}\right), \\
& \zeta_{\mathrm{L}}^{\mathrm{C}-\mathrm{S}}:=-\left\|\tilde{\boldsymbol{\sigma}}^{\mathrm{d}, \mathrm{e}}\right\|_{\sigma}\left\|\tilde{\boldsymbol{\sigma}}^{\mathrm{e}}\right\|_{\sigma}+R\left(\tilde{\mathbf{u}}^{\mathrm{d}}\right) .
\end{aligned}
$$

The use of the Cauchy-Schwarz inequality is typically inducing a large overestimation of the quantities assessed. This is because the two vectors $\tilde{\boldsymbol{\sigma}}^{\mathrm{d}, \mathrm{e}}$ and $\mathbf{s}^{\nu}(\mathbf{e})$ are, in general, far of being parallel. This makes the error bounds given in (76) not sharp, with an unrealistic and impractical bound gap. 


\subsubsection{Bounds using symmetric error equations}

Alternative error bounds, based on different algebraic identities, are often used in the literature to derive sharper bounds than the ones obtained with the Cauchy-Schwarz approach. For instance, the parallelogram identity is used in $[7,65,66]$ in the context of linear elasticity and in $[21,22]$ for transient convection-diffusion-reaction equations. In the framework of structural dynamics reference [46] proposes a bounding expression, alternative to the one in equation (76) originally proposed in reference [45].

The derivation of the alternative bounds requires introducing symmetrized equations for the original and adjoint errors. However, it is worth noting that this is only a mathematical artifact and, in practice, the error bounds are computed using only the admissible pairs $(\tilde{\mathbf{u}}, \tilde{\boldsymbol{\sigma}})$ and $\left(\tilde{\mathbf{u}}^{\mathrm{d}}, \tilde{\boldsymbol{\sigma}}^{\mathrm{d}}\right)$ without solving any auxiliary symmetrized error equations.

The symmetrized error equations read: find $\mathbf{e}^{\nu} \in \mathcal{U}_{0}$ and $\mathbf{e}^{\mathrm{d}, \nu} \in \mathcal{U}_{0}^{\mathrm{d}}$ such that

$$
\begin{gathered}
B^{\nu}\left(\mathbf{e}^{\nu}, \mathbf{w}\right)=R(\mathbf{w}) \quad \forall \mathbf{w} \in \mathcal{W}, \\
B^{\nu}\left(\mathbf{e}^{\mathrm{d}, \nu}, \mathbf{w}\right)=R^{\mathrm{d}}(\mathbf{w}) \quad \forall \mathbf{w} \in \mathcal{W},
\end{gathered}
$$

where the spaces $\mathcal{U}_{0}$ and $\mathcal{U}_{0}^{\mathrm{d}}$ are defined respectively as

$$
\mathcal{U}_{0}:=\{\mathbf{w} \in \mathcal{W}: \mathbf{w}=0 \text { at } \Omega \times\{0\} \text { and } \dot{\mathbf{w}}=0 \text { at } \Omega \times\{0\}\}
$$

and

$$
\mathcal{U}_{0}^{\mathrm{d}}:=\{\mathbf{w} \in \mathcal{W}: \mathbf{w}=0 \text { at } \Omega \times\{T\} \text { and } \dot{\mathbf{w}}=0 \text { at } \Omega \times\{T\}\} .
$$

Equations (77) resemble the residual equation (18) for the primal error e. Note that the difference is that the non symmetric bilinear form $B(\cdot, \cdot)$ is replaced by the symmetric one $B^{\nu}(\cdot, \cdot)$ defined as

$$
B^{\nu}(\mathbf{v}, \mathbf{w}):=a_{2} \int_{I} a(\dot{\mathbf{v}}, \dot{\mathbf{w}}) \mathrm{d} t
$$

It is easily shown that for any scalar value $\kappa \neq 0$, see [46], the following algebraic identity holds:

$$
-\frac{1}{4}\left\|\kappa \mathbf{e}^{\nu}-\frac{1}{\kappa} \mathbf{e}^{\mathrm{d}, \nu}\right\|^{2} \leq R^{\mathrm{d}}(\mathbf{e}) \leq \frac{1}{4}\left\|\kappa \mathbf{e}^{\nu}+\frac{1}{\kappa} \mathbf{e}^{\mathrm{d}, \nu}\right\|^{2} .
$$

Functions $\kappa \mathbf{e}^{\nu} \pm \frac{1}{\kappa} \mathbf{e}^{\mathrm{d}, \nu}$ are solutions of the infinite dimensional problems (77). Therefore, the error bounds proposed in (78) are not computable. However, introducing the constitutive relation errors of the original and adjoint problem, the computable bounds for $\left\|\kappa \mathbf{e}^{\nu} \pm \frac{1}{\kappa} \mathbf{e}^{\mathrm{d}, \nu}\right\|$ are

$$
\left\|\kappa \mathbf{e}^{\nu} \pm \frac{1}{\kappa} \mathbf{e}^{\mathrm{d}, \nu}\right\| \leq\left\|\kappa \tilde{\boldsymbol{\sigma}}^{\mathrm{e}} \pm \frac{1}{\kappa} \tilde{\boldsymbol{\sigma}}^{\mathrm{d}, \mathrm{e}}\right\|_{\sigma} .
$$

The final bounds for $L^{\mathcal{O}}(\mathbf{e})$ are derived substituting expression (79) in equation (78) and adding the correction term $R\left(\tilde{\mathbf{u}}^{\mathrm{d}}\right)$

$$
\begin{aligned}
\zeta_{\mathrm{U}} & :=\frac{1}{4}\left\|\kappa \tilde{\boldsymbol{\sigma}}^{\mathrm{e}}+\frac{1}{\kappa} \tilde{\boldsymbol{\sigma}}^{\mathrm{d}, \mathrm{e}}\right\|_{\sigma}^{2}+R\left(\tilde{\mathbf{u}}^{\mathrm{d}}\right), \\
\zeta_{\mathrm{L}} & :=-\frac{1}{4}\left\|\kappa \tilde{\boldsymbol{\sigma}}^{\mathrm{e}}-\frac{1}{\kappa} \tilde{\boldsymbol{\sigma}}^{\mathrm{d}, \mathrm{e}}\right\|_{\sigma}^{2}+R\left(\tilde{\mathbf{u}}^{\mathrm{d}}\right),
\end{aligned}
$$


where $\zeta_{\mathrm{L}}$ and $\zeta_{\mathrm{U}}$ are such that

$$
\zeta_{\mathrm{L}} \leq L^{\mathcal{O}}(\mathbf{e}) \leq \zeta_{\mathrm{U}}
$$

The error bounds (80) have a similar (but not identical) structure as the ones obtained using the parallelogram rule in linear elasticity. In both cases, the error in the quantity of interest is expressed in terms of energy measures of linear combinations of the original and adjoint errors. The main difference with respect to the parallelogram approach is that, here, energy-like lower bounds of the error are not used to obtain sharper bounds for the quantity of interest.

Note that the bounds (80) hold for any non-zero scalar parameter $\kappa$. In practice, the parameter $\kappa$ is determined such that it minimizes the bound gap, yielding the optimal value

$$
\kappa=\left(\frac{\left\|\tilde{\boldsymbol{\sigma}}^{\mathrm{d}, \mathrm{e}}\right\|_{\sigma}}{\left\|\tilde{\boldsymbol{\sigma}}^{\mathrm{e}}\right\|_{\sigma}}\right)^{1 / 2} .
$$

The error bounds $\zeta_{\mathrm{U}}, \zeta_{\mathrm{L}}$ proposed in (80) are sharper than $\zeta_{\mathrm{U}}^{\mathrm{C}-\mathrm{S}}, \zeta_{\mathrm{L}}^{\mathrm{C}-\mathrm{S}}$ in (76) obtained using the Cauchy-Schwartz inequality. Indeed, introducing the optimal value of $\kappa$ given by (81) into the bound expression (80) yields

$$
\begin{aligned}
& \zeta_{\mathrm{U}}=\frac{1}{2}\left\|\tilde{\boldsymbol{\sigma}}^{\mathrm{d}, \mathrm{e}}\right\|_{\sigma}\left\|\tilde{\boldsymbol{\sigma}}^{\mathrm{e}}\right\|_{\sigma}+R\left(\tilde{\mathbf{u}}^{\mathrm{d}}\right)+\frac{1}{2} \bar{B}^{\nu}\left(\tilde{\boldsymbol{\sigma}}^{\mathrm{d}, \mathrm{e}}, \tilde{\boldsymbol{\sigma}}^{\mathrm{e}}\right), \\
& \zeta_{\mathrm{L}}=-\frac{1}{2}\left\|\tilde{\boldsymbol{\sigma}}^{\mathrm{d} \mathrm{e} \mathrm{e}}\right\|_{\sigma}\left\|\tilde{\boldsymbol{\sigma}}^{\mathrm{e}}\right\|_{\sigma}+R\left(\tilde{\mathbf{u}}^{\mathrm{d}}\right)+\frac{1}{2} \bar{B}^{\nu}\left(\tilde{\boldsymbol{\sigma}}^{\mathrm{d}, \mathrm{e}}, \tilde{\boldsymbol{\sigma}}^{\mathrm{e}}\right) .
\end{aligned}
$$

The bound gap, that is the difference between the upper and lower bound, is therefore $\zeta_{\mathrm{U}}-\zeta_{\mathrm{L}}=\left\|\tilde{\boldsymbol{\sigma}}^{\mathrm{d}, \mathrm{e}}\right\|_{\sigma}\left\|\tilde{\boldsymbol{\sigma}}^{\mathrm{e}}\right\|_{\sigma}$ whereas the bound gap of the bounds in equation (76) is $\zeta_{\mathrm{U}}^{\mathrm{C}-\mathrm{S}}-$ $\zeta_{\mathrm{L}}^{\mathrm{C}-\mathrm{S}}=2\left\|\tilde{\boldsymbol{\sigma}}^{\mathrm{d}, \mathrm{e}}\right\|_{\sigma}\left\|\tilde{\boldsymbol{\sigma}}^{\mathrm{e}}\right\|_{\sigma}$. Hence, the bound gap in equation (80) is half of the bound gap corresponding to equation $(76)$, that is $\zeta_{\mathrm{U}}-\zeta_{\mathrm{L}}=\frac{1}{2}\left(\zeta_{\mathrm{U}}^{\mathrm{C}-\mathrm{S}}-\zeta_{\mathrm{L}}^{\mathrm{C}-\mathrm{S}}\right)$, and provides a sharper error assessment.

\subsubsection{Equivalent alternative approach}

The error bounds (82) are derived here using an alternative presentation, without introducing the symmetrized error equations and following a rationale similar to the one presented in references $[67,68]$ for steady-state linear elasticity. This alternative approach requires introducing an auxiliary stress field that stands for the error with respect to the averaged viscous stress, namely

$$
\boldsymbol{\sigma}_{\text {ave }}^{\mathrm{e}, \nu}:=\boldsymbol{\sigma}^{\nu}-\frac{1}{2}\left(\tilde{\boldsymbol{\sigma}}^{\nu}+\mathbf{s}^{\nu}(\tilde{\mathbf{u}})\right) .
$$

Note that $\boldsymbol{\sigma}_{\text {ave }}^{\mathrm{e}, \nu}$ is introduced as a mathematical artifact (it is not computable because it involves the exact solution $\mathbf{u})$ allowing to rewrite the residual $R^{\mathrm{d}}(\mathbf{e})$ as

$$
R^{\mathrm{d}}(\mathbf{e})=\bar{B}^{\nu}\left(\tilde{\boldsymbol{\sigma}}^{\mathrm{d}, \mathrm{e}}, \mathbf{s}^{\nu}(\mathbf{e})\right)=\bar{B}^{\nu}\left(\tilde{\boldsymbol{\sigma}}^{\mathrm{d}, \mathrm{e}}, \boldsymbol{\sigma}_{\mathrm{ave}}^{\mathrm{e}, \nu}\right)+\frac{1}{2} \bar{B}^{\nu}\left(\tilde{\boldsymbol{\sigma}}^{\mathrm{d}, \mathrm{e}}, \tilde{\boldsymbol{\sigma}}^{\mathrm{e}}\right)
$$


Hence, the bounds for $R^{\mathrm{d}}(\mathbf{e})$ are obtained bounding the value $\bar{B}^{\nu}\left(\tilde{\boldsymbol{\sigma}}^{\mathrm{d}, \mathrm{e}}, \boldsymbol{\sigma}_{\text {ave }}^{\mathrm{e}, \nu}\right)$ which is the only non-computable term in the right hand side of equation (84). The computable bound for $\bar{B}^{\nu}\left(\tilde{\boldsymbol{\sigma}}^{\mathrm{d}, \mathrm{e}}, \boldsymbol{\sigma}_{\text {ave }}^{\mathrm{e}, \nu}\right)$ is derived by applying the Cauchy-Schwartz inequality

$$
\left|\bar{B}^{\nu}\left(\tilde{\boldsymbol{\sigma}}^{\mathrm{d}, \mathrm{e}}, \tilde{\boldsymbol{\sigma}}_{\text {ave }}^{\mathrm{e}}\right)\right| \leq\left\|\tilde{\boldsymbol{\sigma}}^{\mathrm{d}, \mathrm{e}}\right\|_{\sigma}\left\|\tilde{\boldsymbol{\sigma}}_{\text {ave }}^{\mathrm{e}, \nu}\right\|_{\sigma},
$$

and then bounding $\left\|\tilde{\boldsymbol{\sigma}}_{\text {ave }}^{\mathrm{e}, \nu}\right\|_{\sigma}$. The following theorem proves that the constitutive relation error $\tilde{\boldsymbol{\sigma}}^{\mathrm{e}}$ provides a bound for $\left\|\tilde{\boldsymbol{\sigma}}_{\text {ave }}^{\mathrm{e}, \nu}\right\|_{\sigma}$.

Theorem 3. The constitutive relation error $\tilde{\boldsymbol{\sigma}}^{\mathrm{e}}$ defined in equation (70) leads to the following upper bound of the averaged stress error $\boldsymbol{\sigma}_{\text {ave }}^{\mathrm{e}, \nu}$ defined in equation (83),

$$
\frac{1}{2}\left\|\tilde{\boldsymbol{\sigma}}^{\mathrm{e}}\right\|_{\sigma} \geq\left\|\boldsymbol{\sigma}_{\mathrm{ave}}^{\mathrm{e}, \nu}\right\|_{\sigma} .
$$

Proof. Using the relation $\boldsymbol{\sigma}_{\text {ave }}^{\mathrm{e}, \nu}=\mathbf{s}^{\nu}(\mathbf{e})-\frac{1}{2} \tilde{\boldsymbol{\sigma}}^{\mathrm{e}}$, the value $\left\|\boldsymbol{\sigma}_{\text {ave }}^{\mathrm{e}, \nu}\right\|_{\sigma}^{2}$ is rewritten as

$$
\begin{aligned}
\left\|\boldsymbol{\sigma}_{\text {ave }}^{\mathrm{e}, \nu}\right\|_{\sigma}^{2} & =\frac{1}{4}\left\|\tilde{\boldsymbol{\sigma}}^{\mathrm{e}}\right\|_{\sigma}^{2}+\left\|\mathbf{s}^{\nu}(\mathbf{e})\right\|_{\sigma}^{2}-\bar{B}^{\nu}\left(\mathbf{s}^{\nu}(\mathbf{e}), \tilde{\boldsymbol{\sigma}}^{\mathrm{e}}\right), \\
& =\frac{1}{4}\left\|\tilde{\boldsymbol{\sigma}}^{\mathrm{e}}\right\|_{\sigma}^{2}+\bar{B}^{\nu}\left(\mathbf{s}^{\nu}(\mathbf{e}), \mathbf{s}^{\nu}(\mathbf{e})-\tilde{\boldsymbol{\sigma}}^{\mathrm{e}}\right), \\
& =\frac{1}{4}\left\|\tilde{\boldsymbol{\sigma}}^{\mathrm{e}}\right\|_{\sigma}^{2}+\bar{B}^{\nu}\left(\mathbf{s}^{\nu}(\mathbf{e}), \boldsymbol{\sigma}^{\nu}-\tilde{\boldsymbol{\sigma}}^{\nu}\right) .
\end{aligned}
$$

The proof is concluded noting that $\bar{B}^{\nu}\left(\mathbf{s}^{\nu}(\mathbf{e}), \boldsymbol{\sigma}^{\nu}-\tilde{\boldsymbol{\sigma}}^{\nu}\right) \leq 0$, which directly proves that (86) holds.

The statement $\bar{B}^{\nu}\left(\mathbf{s}^{\nu}(\mathbf{e}), \boldsymbol{\sigma}^{\nu}-\tilde{\boldsymbol{\sigma}}^{\nu}\right) \leq 0$ is proved noting that, for an admissible pair $(\tilde{\mathbf{u}}, \tilde{\boldsymbol{\sigma}}) \in \mathcal{U} \times \mathcal{S}(\tilde{\mathbf{u}})$, the following relation holds

$$
0=\int_{I}(\rho(\ddot{\mathbf{u}}-\ddot{\hat{\mathbf{u}}}), \dot{\mathbf{e}}) \mathrm{d} t+\int_{I}(\boldsymbol{\sigma}-\tilde{\boldsymbol{\sigma}}, \boldsymbol{\varepsilon}(\dot{\mathbf{e}})) \mathrm{d} t .
$$

Then, injecting the expression

$$
\boldsymbol{\sigma}-\tilde{\boldsymbol{\sigma}}=\mathbf{s}^{\mathrm{E}}(\mathbf{u}-\tilde{\mathbf{u}})+\boldsymbol{\sigma}^{\nu}-\tilde{\boldsymbol{\sigma}}^{\nu}
$$

into equation (87) one has

$$
\begin{aligned}
0 & =\int_{I}(\rho \ddot{\mathbf{e}}, \dot{\mathbf{e}}) \mathrm{d} t+\int_{I} a(\mathbf{e}, \dot{\mathbf{e}}) \mathrm{d} t+\bar{B}^{\nu}\left(\mathbf{s}^{\nu}(\mathbf{e}), \boldsymbol{\sigma}^{\nu}-\tilde{\boldsymbol{\sigma}}^{\nu}\right) \\
& =\frac{1}{2} \int_{I} \frac{\mathrm{d}}{\mathrm{d} t}(\rho \dot{\mathbf{e}}, \dot{\mathbf{e}}) \mathrm{d} t+\frac{1}{2} \int_{I} \frac{\mathrm{d}}{\mathrm{d} t} a(\mathbf{e}, \mathbf{e}) \mathrm{d} t+\bar{B}^{\nu}\left(\mathbf{s}^{\nu}(\mathbf{e}), \boldsymbol{\sigma}^{\nu}-\tilde{\boldsymbol{\sigma}}^{\nu}\right) \\
& =\frac{1}{2}\left[\|\dot{\mathbf{e}}\|_{m}^{2}+\|\mathbf{e}\|_{a}^{2}\right]_{t=0}^{t=T}+\bar{B}^{\nu}\left(\mathbf{s}^{\nu}(\mathbf{e}), \boldsymbol{\sigma}^{\nu}-\tilde{\boldsymbol{\sigma}}^{\nu}\right) .
\end{aligned}
$$

Taking into account that $\mathbf{e}(0)=\dot{\mathbf{e}}(0)=\mathbf{0}$, one has

$$
\bar{B}^{\nu}\left(\mathbf{s}^{\nu}(\mathbf{e}), \boldsymbol{\sigma}^{\nu}-\tilde{\boldsymbol{\sigma}}^{\nu}\right)=-\frac{1}{2}\|\dot{\mathbf{e}}(T)\|_{m}^{2}-\frac{1}{2}\|\mathbf{e}(T)\|_{a}^{2},
$$

which proves that $\bar{B}^{\nu}\left(\mathbf{s}^{\nu}(\mathbf{e}), \boldsymbol{\sigma}^{\nu}-\tilde{\boldsymbol{\sigma}}^{\nu}\right) \leq 0$. 
Using equations (85) and (86), the computable bound for $\left|\bar{B}^{\nu}\left(\tilde{\boldsymbol{\sigma}}^{\mathrm{d}, \mathrm{e}}, \tilde{\boldsymbol{\sigma}}_{\text {ave }}^{\mathrm{e}}\right)\right|$ is readily obtained as

$$
\left|\bar{B}^{\nu}\left(\tilde{\boldsymbol{\sigma}}^{\mathrm{d}, \mathrm{e}}, \tilde{\boldsymbol{\sigma}}_{\text {ave }}^{\mathrm{e}}\right)\right| \leq \frac{1}{2}\left\|\tilde{\boldsymbol{\sigma}}^{\mathrm{d}, \mathrm{e}}\right\|_{\sigma}\left\|\tilde{\boldsymbol{\sigma}}^{\mathrm{e}}\right\|_{\sigma} .
$$

Using equation (88) together with equations (84) and (37), the result given in equation (82) is derived in an alternative way, without using the symmetrized error equations.

\subsection{Construction of D-admissible fields}

This section describes in detail the computation of a D-admissible stress $\tilde{\boldsymbol{\sigma}} \in \mathcal{S}(\tilde{\mathbf{u}})$, given a K-admissible field $\tilde{\mathbf{u}} \in \mathcal{U}$. The presentation focuses in the original problem because the same methodology is used also for the adjoint problem.

The stress $\tilde{\boldsymbol{\sigma}} \in \mathcal{S}(\tilde{\mathbf{u}})$ is characterized by a series of stresses $\tilde{\boldsymbol{\sigma}}_{n}, n=0, \ldots, N$ at the time points in $\mathcal{T}$. Each $\tilde{\boldsymbol{\sigma}}_{n}$ is seen as a statically equilibrated stress field for some loading. Thus, the D-admissible stress $\tilde{\boldsymbol{\sigma}}$ is eventually computed solving a series of static equilibration problems following the standard procedures described in $[9,8,66]$.

The following theorem demonstrates how the the D-admissible stress $\tilde{\boldsymbol{\sigma}} \in \mathcal{S}(\tilde{\mathbf{u}})$ can be computed in terms of the statically equilibrated stresses $\tilde{\boldsymbol{\sigma}}_{n}, n=0, \ldots, N$.

Theorem 4. Given the external loads $\mathbf{f}, \mathbf{g}$ and a K-admissible field $\tilde{\mathbf{u}} \in \mathcal{U}$, then a $D$-admissible stress $\tilde{\boldsymbol{\sigma}} \in \mathcal{S}(\tilde{\mathbf{u}})$ is straightforwardly defined through piecewise linear interpolation in time

$$
\tilde{\boldsymbol{\sigma}}(\mathbf{x}, t):=\sum_{n=0}^{N} \tilde{\boldsymbol{\sigma}}_{n}(\mathbf{x}) \theta_{n}(t),
$$

provided that: 1) the stress fields $\tilde{\boldsymbol{\sigma}}_{n}, n=0, \ldots, N$ fulfill the static equilibrium condition

$$
\left(\tilde{\boldsymbol{\sigma}}_{n}, \boldsymbol{\varepsilon}(\mathbf{w})\right)=l_{n}(\mathbf{w})-\left(\rho \ddot{\tilde{\mathbf{u}}}_{n}, \mathbf{w}\right) \quad \forall \mathbf{w} \in \mathcal{V}_{0},
$$

or equivalently

$$
\begin{aligned}
\boldsymbol{\nabla} \cdot \tilde{\boldsymbol{\sigma}}_{n} & =\mathbf{f}_{n}-\rho \ddot{\tilde{\mathbf{u}}}_{n} \quad \text { in } \Omega^{\mathrm{int}}, \\
\llbracket \tilde{\boldsymbol{\sigma}}_{n} \cdot \mathbf{n} \rrbracket & =\mathbf{0} \quad \text { on } \Gamma^{\mathrm{int}}, \\
\tilde{\boldsymbol{\sigma}}_{n} \cdot \mathbf{n} & =\mathbf{g}_{n} \quad \text { on } \Gamma_{\mathrm{N}},
\end{aligned}
$$

and 2) the external loads $\mathbf{f}, \mathbf{g}$ and the acceleration $\ddot{\tilde{\mathbf{u}}}$ are piecewise linear in time, i.e.

$$
\begin{aligned}
& \mathbf{f}(\mathbf{x}, t)=\sum_{n=0}^{N} \mathbf{f}_{n}(\mathbf{x}) \theta_{n}(t), \\
& \mathbf{g}(\mathbf{x}, t)=\sum_{n=0}^{N} \mathbf{g}_{n}(\mathbf{x}) \theta_{n}(t), \\
& \ddot{\tilde{\mathbf{u}}}(\mathbf{x}, t)=\sum_{n=0}^{N} \ddot{\tilde{\mathbf{u}}}_{n}(\mathbf{x}) \theta_{n}(t) .
\end{aligned}
$$


Most of the techniques providing D-admissible stresses, see $[9,8,66]$, require as input an approximation of the stresses, say $\boldsymbol{\sigma}_{n}^{H, \Delta t} \approx \boldsymbol{\sigma}\left(t_{n}\right)$, fulfilling a discrete form of (90), namely

$$
\left(\rho \ddot{\tilde{\mathbf{u}}}_{n}, \mathbf{w}\right)+\left(\boldsymbol{\sigma}_{n}^{H, \Delta t}, \boldsymbol{\varepsilon}(\mathbf{w})\right)=l_{n}(\mathbf{w}) \quad \forall \mathbf{w} \in \mathcal{V}_{0}^{H},
$$

being $\mathcal{V}_{0}^{H}$ the usual functional space associated with the computational mesh. This relation guarantees that the local problems are solvable.

Note that equation (93) holds defining the admissible solution $\tilde{\mathbf{u}}$ as in (7), that is $\ddot{\tilde{\mathbf{u}}}_{n}=$ $\mathbf{a}_{n}^{H, \Delta t}$, and taking discrete stress as $\boldsymbol{\sigma}_{n}^{H, \Delta t}:=\mathcal{C}: \boldsymbol{\varepsilon}\left(\mathbf{u}_{n}^{H, \Delta t}+a_{2} \mathbf{v}_{n}^{H, \Delta t}\right.$ ) (being $\mathbf{u}_{n}^{H, \Delta t}$ and $\mathbf{v}_{n}^{H, \Delta t}$ the Newmark displacements and velocities).

\subsubsection{The hybrid fluxes method}

The hybrid fluxes method introduced by Ladevèze in [2] is a classical stress equilibration technique. It is also denoted in more recent works by EET (Element Equilibration Technique). This methodology provides stress fields $\tilde{\boldsymbol{\sigma}}_{n}, n=0, \ldots, N$, fulfilling equations (90) and (91). The construction of the equilibrated stress field $\tilde{\boldsymbol{\sigma}}_{n}$ is based on some approximate stress $\boldsymbol{\sigma}_{n}^{H, \Delta t}$ that is taken as the input of the procedure. This section is devoted to present this methodology, stressing the technical details of its application to compute D-admissible stresses.

Some additional notations are needed to introduce the hybrid fluxes method. The location of a generic node of the computational mesh is denoted by $\mathbf{x}_{i}, i=1, \ldots, N_{\text {no }}$, being $N_{\text {no }}$ the total number of nodes. As introduced before, elements in the mesh are denoted by $\Omega_{k} \subset \Omega, k=1, \ldots, N_{\mathrm{el}}$, where $N_{\mathrm{el}}$ is the total number of elements. Element sides (or faces in 3D) are denoted by $\Gamma_{l} \subset \bar{\Omega}, l=1, \ldots, N_{\mathrm{fa}}$, being $N_{\mathrm{fa}}$ their total number (note that $\Gamma_{l}$ is either an inter-element boundary, that is $\Gamma_{l}=\partial \Omega_{k} \bigcap \partial \Omega_{k^{\prime}}$ for some $k$ and $k^{\prime}$ or a boundary element side, that is $\Gamma_{l}=\partial \Omega_{k} \bigcap \partial \Omega$ for some $k$ ). Also, some sets of indices are introduced describing the connectivity of every node $\mathbf{x}_{i}$, element $\Omega_{k}$ and face $\Gamma_{l}$. The set $\mathcal{N}\left(\Omega_{k}\right)$ is the standard connectivity information containing the indices of the nodes of element $\Omega_{k}$. The set $\mathcal{E}\left(\mathbf{x}_{i}\right)$ contains the indices of the elements to which node $\mathbf{x}_{i}$ belongs. The set $\mathcal{F}\left(\mathbf{x}_{i}\right)$ contains the indices of the sides/faces to which node $\mathbf{x}_{i}$ belongs. The set $\mathcal{F}\left(\Omega_{k}\right)$ contains the indices of the faces of element $\Omega_{k}$. Finally, the set $\mathcal{N}\left(\Gamma_{l}\right)$ contains the indices of the nodes of face $\Gamma_{l}$. Figure 8 illustrates the definitions of these sets.

The equilibrated stresses $\tilde{\boldsymbol{\sigma}}_{n}$ at time $t_{n}, n=0, \ldots, N$, is computed solving local equilibrium problems element-by-element. Each local problem consists in finding the restriction of $\tilde{\boldsymbol{\sigma}}_{n}$ to element $\Omega_{k}$ of the mesh, $k=1, \ldots, N_{\mathrm{el}}$, such that

$$
\begin{aligned}
\nabla \cdot \tilde{\boldsymbol{\sigma}}_{n} & =\mathbf{f}_{n}-\rho \ddot{\tilde{\mathbf{u}}}_{n} \quad \text { in } \Omega_{k}, \\
\tilde{\boldsymbol{\sigma}}_{n} \cdot \mathbf{n}_{k} & =\eta_{k l} \mathbf{t}_{n} \quad \text { on } \partial \Gamma_{l} \subset \Omega_{k} .
\end{aligned}
$$

It is worth noting that the boundary conditions (94b) for the local problem (94) are not known and they require obtaining the inter-element tractions $\mathbf{t}_{n}$, defined on every $\Gamma_{l}$, for $l=1, \ldots, N_{\mathrm{fa}}$. The coefficient $\eta_{k l}$ takes the values 1 or -1 , depending on the orientation of the face $\Gamma_{l}$ with respect to $\Omega_{k}$. It is assumed that the orientation of $\Gamma_{l}$ is given by a 

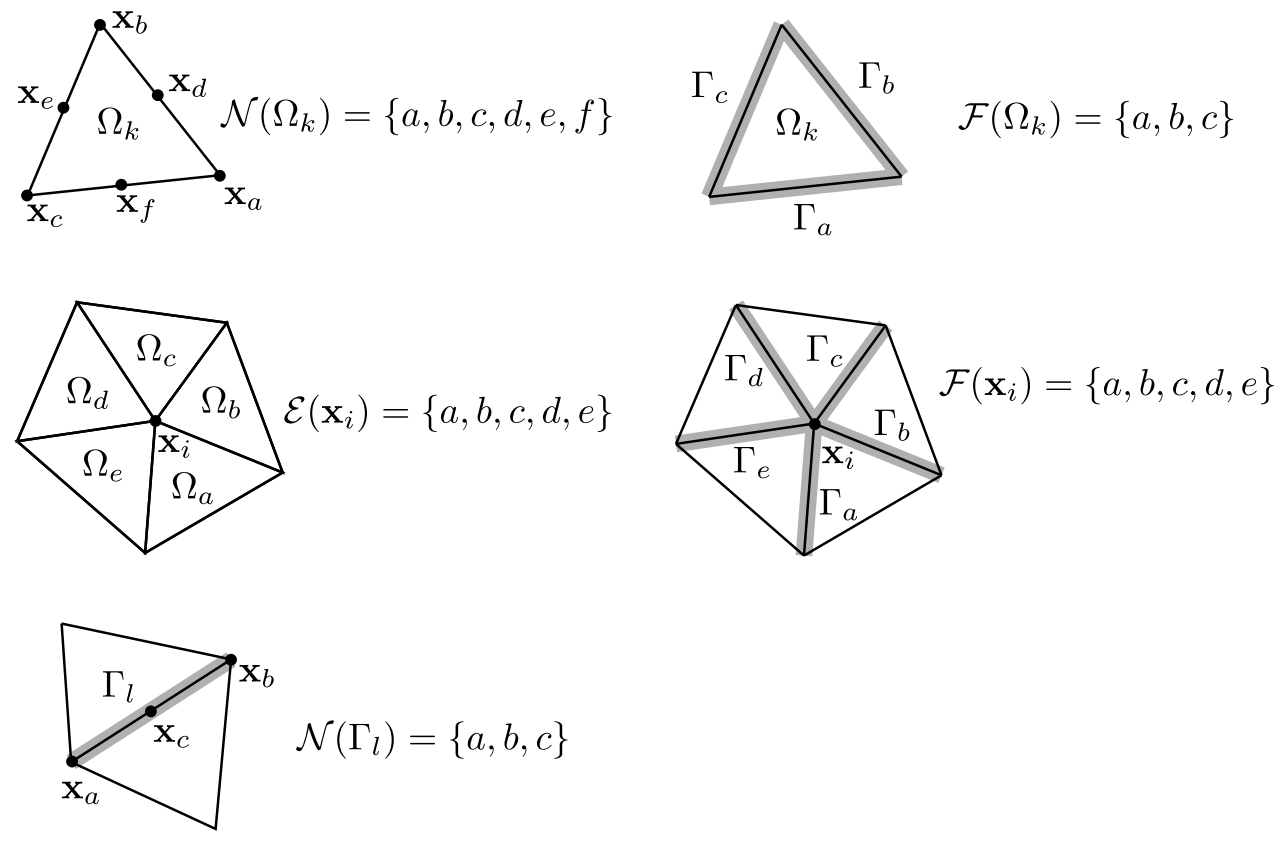

Figure 8: Illustration of sets $\mathcal{N}\left(\Omega_{k}\right), \mathcal{F}\left(\Omega_{k}\right), \mathcal{E}\left(\mathbf{x}_{i}\right), \mathcal{F}\left(\mathbf{x}_{i}\right)$ and $\mathcal{N}\left(\Gamma_{l}\right)$.

normal unit vector $\tilde{\mathbf{n}}_{l}$ and then $\eta_{k l}=\tilde{\mathbf{n}}_{l} \cdot \mathbf{n}_{k}$. Moreover, equation(94) is a pure Neumann problem and therefore is only well posed if the prescribed loads, the body forces and the tractions in the right-hand-sides of (94a) and (94b), are in equilibrium.

Thus, the inter-element tractions $\mathbf{t}_{n}$ must be computed previous to solving the local problems (94) and they must fulfill local equilibrium, namely

$$
\sum_{l \in \mathcal{F}\left(\Omega_{k}\right)} \int_{\Gamma_{l}} \eta_{k l} \mathbf{t}_{n} \cdot \mathbf{w} \mathrm{d} \Gamma+\int_{\Omega_{k}}\left(\mathbf{f}_{n}-\rho \ddot{\tilde{\mathbf{u}}}_{n}\right) \cdot \mathbf{w} \mathrm{d} \Omega=0,
$$

for all $\mathbf{w}$ in the space of rigid body motions. This space is defined as (in 3D) $\operatorname{span}\left\{(1,0,0)^{T},(0,1,0)^{T},(0,0,1)^{T},(-y, x, 0)^{T},(-z, 0, x)^{T},(0,-z, y)^{T}\right\}$.

On the faces on the Neumann boundary, that is for $\Gamma_{l} \subset \Gamma_{N}$, the tractions have to match the actual boundary conditions (91c), that is $\eta_{k l} \mathbf{t}_{n}=\mathbf{g}_{n}$.

At the first sight, obtaining the inter-element tractions $\mathbf{t}_{n}$ fulfilling (95) leads to a global problem and requires solving the unknowns for all the faces $\Gamma_{l}$, for $l=1, \ldots, N_{\mathrm{fa}}$, resulting in a large system of linear equations. In practice, this problem is decoupled into local computations thanks to the idea introduced in [2], which is based on enforcing locally (for each element $\Omega_{k}$ ) the so-called prolongation condition

$$
\int_{\Omega_{k}}\left(\tilde{\boldsymbol{\sigma}}_{n}-\boldsymbol{\sigma}_{n}^{H, \Delta t}\right) \cdot \nabla \varphi_{i} \mathrm{~d} \Omega=\mathbf{0} \quad \forall i \in \mathcal{N}\left(\Omega_{k}\right),
$$

where $\varphi_{i}$ is the shape function associated with node $\mathbf{x}_{i}$. Note that this additional restriction is selecting a particular solution for $\mathbf{t}_{n}$ and hence of $\tilde{\boldsymbol{\sigma}}_{n}$. The problem (95) is decoupled into local computations precisely for this particular solution. 
Assuming that $\boldsymbol{\sigma}^{H, \Delta t}$ is such that (93) holds, enforcing the prolongation condition (96) is equivalent to find $\mathbf{t}_{n}$ such that

$$
\sum_{l \in \mathcal{F}\left(\Omega_{k}\right)} \int_{\Gamma_{l}} \eta_{k l} \mathbf{t}_{n} \varphi_{i} \mathrm{~d} \Gamma=\int_{\Omega_{k}}\left(\boldsymbol{\sigma}_{n}^{H, \Delta t} \cdot \nabla \varphi_{i}-\left(\mathbf{f}_{n}-\rho \ddot{\tilde{\mathbf{u}}}_{n}\right) \varphi_{i}\right) \mathrm{d} \Omega,
$$

for all mesh elements $\Omega_{k}, k=1, \ldots, N_{\mathrm{el}}$ and for all nodes $i \in \mathcal{N}\left(\Omega_{k}\right)$, pertaining to element $\Omega_{k}$.

If $\mathbf{t}_{n}$ fulfills (97) and (93) holds, then the equilibrium condition (95) is satisfied and $\tilde{\boldsymbol{\sigma}}_{n}$ can be computed solving the local problems (94). Thus, the tractions $\mathbf{t}_{n}$ are obtained such that they fulfill equation (97).

The following definitions are introduced:

$$
\mathbf{b}_{i l}:=\int_{\Gamma_{l}} \mathbf{t}_{n} \varphi_{i} \mathrm{~d} \Gamma \quad \text { and } \quad \mathbf{j}_{k i}:=\int_{\Omega_{k}}\left(\boldsymbol{\sigma}_{n}^{H, \Delta t} \cdot \nabla \varphi_{i}-\left(\mathbf{f}_{n}-\rho \ddot{\tilde{\mathbf{u}}}_{n}\right) \varphi_{i}\right) \mathrm{d} \Omega .
$$

Note that $\mathbf{b}_{i l}$ is nonzero only if $l \in \mathcal{F}\left(\mathbf{x}_{i}\right)$ or, conversely, if $i \in \mathcal{N}\left(\Gamma_{l}\right)$. Thus, equation (97) yields

$$
\sum_{l \in\left(\mathcal{F}\left(\Omega_{k}\right) \cap \mathcal{F}\left(\mathbf{x}_{i}\right)\right)} \eta_{k l} \mathbf{b}_{i l}=\mathbf{j}_{k i}
$$

for all mesh elements $\Omega_{k}, k=1, \ldots, N_{\mathrm{el}}$ and for all nodes $i \in \mathcal{N}\left(\Omega_{k}\right)$.

Expression (99) is a linear system of vectorial equations (vectorial, in the sense that $\mathbf{b}_{i l}$ and $\mathbf{j}_{k i}$ are vectors). The number of vectorial equations is equal to the number of elements $N_{\text {el }}$ times the number of element nodes (i.e. three for linear triangles). The unknowns are the values $\mathbf{b}_{i l}$, for $l=1, \ldots, N_{\mathrm{fa}}$ and $i \in \mathcal{N}\left(\Gamma_{l}\right)$, which are the projections of the traction $\mathbf{t}_{n}$ in the FE functional space (restricted to the faces). The number of unknowns is $N_{\mathrm{fa}}$ times the number of edge/face nodes (i.e. two for linear triangles). The number of unknowns is typically larger than the number of equations, and therefore, additional criteria are required to select one of the solutions.

Remark 5. For the sake of illustration, the equations and unknowns accounting is performed for linear $2 D$ triangles in the case of a Dirichlet problem. The number of equations in (99) is $N_{\mathrm{eq}}=3 N_{\mathrm{el}}$ and the number of unknowns is $N_{\mathrm{unk}}=2 N_{\mathrm{fa}}$. The number of mesh faces is expressed in terms of the number of mesh elements as $N_{\mathrm{fa}}=\frac{3}{2} N_{\mathrm{el}}+\frac{1}{2} N_{\partial \Omega}$, where $N_{\partial \Omega}$ is the number of faces on the boundary. Thus, the number of unknowns and equations are such that $N_{\mathrm{unk}}=N_{\mathrm{eq}}+N_{\partial \Omega}>N_{\mathrm{eq}}$.

At first sight, expression (99) leads to a global system of equations, involving the complete computational domain. However, the global system is decoupled into $N_{\text {no }}$ local systems, associated with each node of the mesh, $\mathbf{x}_{i}$, and involving only the unknowns $\mathbf{b}_{i l}$ for $l \in \mathcal{F}\left(\mathbf{x}_{i}\right)$. In other words, the range for $i$ and $k$ in the system of equations (99) is rewritten as: for $i=1, \ldots, N_{\text {no }}$ and then for all $k \in \mathcal{E}\left(\mathbf{x}_{i}\right)$. In that sense, for a given value of $i$, stating (99) for all $k \in \mathcal{E}\left(\mathbf{x}_{i}\right)$ leads to a system of equations involving only the unknowns $\mathbf{b}_{i l}$ for $l \in \mathcal{F}\left(\mathbf{x}_{i}\right)$ which do not participate in any other local system associated 
with a different node. For a given $\mathbf{x}_{i}, i=1, \ldots, N_{\text {no }}$, the local problem is a reformulation of (99) reading

$$
\sum_{l \in\left(\mathcal{F}\left(\Omega_{k}\right) \cap \mathcal{F}\left(\mathbf{x}_{i}\right)\right)} \eta_{k l} \mathbf{b}_{i l}=\mathbf{j}_{k i}, \quad \forall k \in \mathcal{E}\left(\mathbf{x}_{i}\right) .
$$

All the unknown values $\mathbf{b}_{i l}$, for $l=1, \ldots, N_{\mathrm{fa}}$ and $i \in \mathcal{N}\left(\Gamma_{l}\right)$, are determined once the local problems are solved for all mesh nodes $\mathbf{x}_{i}, i=1, \ldots, N_{\text {no }}$.

The actual resolution of the system (100) depends whether the current node is interior or on the boundary, and (for higher order elements) if the node coincides with a mesh vertex or not. See reference [9] for a detailed discussion of all these cases.

For the sake of simplicity, the presentation in detail of one of these local systems is restricted to the particular case of an interior (not on the boundary) node $\mathbf{x}_{i}$, being also an element vertex. In this case, the number of equations in system $(100)$ is $\# \mathcal{E}\left(\mathbf{x}_{i}\right)$ (one for each element in $\mathcal{E}\left(\mathbf{x}_{i}\right)$, \# denotes the cardinal) and the number of unknowns is $\# \mathcal{F}\left(\mathbf{x}_{i}\right)$ (one for each face in $\mathcal{F}\left(\mathbf{x}_{i}\right)$ ). Note that the number of elements in $\mathcal{E}\left(\mathbf{x}_{i}\right)$ coincides with the number of faces in $\mathcal{F}\left(\mathbf{x}_{i}\right)$ because the node $\mathbf{x}_{i}$ is interior. Thus, the local system (100) has the same number of equations and unknowns. The square matrix associated with the local system of equations (100) has entries $\eta_{k l}$ (thus, equal to \pm 1 or equal to 0 if $k \notin \mathcal{E}\left(\Gamma_{l}\right)$ ) and does not have full rank. The rank deficiency is readily shown by summing up all the equations of system (100) (summing up in $k$ ). Note that for a given face $\Gamma_{l}$ there are only two adjacent elements, say $\tilde{k}$ and $\tilde{k}^{\prime}$. Consequently, the resulting equation is

$$
\sum_{l \in \mathcal{F}\left(\mathbf{x}_{i}\right)}\left(\eta_{\tilde{k} l}+\eta_{\tilde{k}^{\prime} l}\right) \mathbf{b}_{i l}=\sum_{k \in \mathcal{E}\left(\mathbf{x}_{i}\right)} \mathbf{j}_{k i},
$$

Note that $\eta_{\tilde{k} l}+\eta_{\tilde{k}^{\prime} l}=0$ and therefore problem (100) is solvable only if the right hand side data fulfills

$$
\sum_{k \in \mathcal{E}\left(\mathbf{x}_{i}\right)} \mathbf{j}_{k i}=\mathbf{0}
$$

The previous requirement is fulfilled if equation (93) holds. In fact, this is a version of the Galerkin orthogonality property. Under this assumption, system (100) is compatible but, due to the rank deficiency, it has infinite solutions. A particular solution is found such that it minimizes the functional

$$
\Phi_{i}\left(\mathbf{b}_{i l}\right):=\frac{1}{2} \sum_{l \in \mathcal{F}\left(\mathbf{x}_{i}\right)}\left(\mathbf{b}_{i l}-\overline{\mathbf{b}}_{i l}\right)^{2}
$$

with

$$
\overline{\mathbf{b}}_{i l}:=\frac{1}{2} \int_{\Gamma_{l}}\left(\left.\boldsymbol{\sigma}_{n}^{H, \Delta t}\right|_{\Omega_{\tilde{k}}}+\left.\boldsymbol{\sigma}_{n}^{H, \Delta t}\right|_{\Omega_{\tilde{k}^{\prime}}}\right) \cdot \tilde{\mathbf{n}}_{l} \varphi_{i} \mathrm{~d} \Gamma .
$$

Once the quantities $\mathbf{b}_{i l}$, for $l=1, \ldots, N_{\mathrm{fa}}$ and $i \in \mathcal{N}\left(\Gamma_{l}\right)$, are available, the tractions $\mathbf{t}_{n}$ are completely determined. In some cases, is it useful to parametrize tractions $\mathbf{t}_{n}$ using nodal values instead of quantities $\mathbf{b}_{i l}$. Specifically, the nodal values parametrizing the 
restriction of $\mathbf{t}_{n}$ to the face $\Gamma_{l}$ are obtained solving a linear system of equations with the mass matrix with entries

$$
\int_{\Gamma_{l}} \varphi_{i} \varphi_{j} \mathrm{~d} \Gamma, \quad i, j \in \mathcal{N}\left(\Gamma_{l}\right)
$$

and the right hand side vector containing the values $\mathbf{b}_{i l}, i \in \mathcal{N}\left(\Gamma_{l}\right)$. For $2 \mathrm{D}$ linear elements, the system to be solved at each element side has two unknowns and two equations.

Once the tractions $\mathbf{t}_{n}$ are available, the stress field $\tilde{\boldsymbol{\sigma}}_{n}$ is obtained solving the local problems (94) in each element $\Omega_{k}$. The local Neumann problems (94) can be solved taking as unknowns either displacements (standard FE approach) or stresses (the so-called dual formulations). The standard displacement-based approach uses a finite element solver locally, selecting a reference mesh (created with $H$ or $p$ refinement) discretizing each element. The local approximate solution undervaluates the energy of the exact solution and therefore the global upper bound property is not strictly guaranteed. The resulting estimates are referred as asymptotic [69] because the upper bound property holds only asymptotically, as the element size of the reference mesh tends to zero (or the degree of the polynomial tents to infinity). Alternatively, the dual approach (taking stresses as unknowns) provides directly D-admissible piecewise polynomial solutions for $\tilde{\boldsymbol{\sigma}}_{n}$. In this case, the upper bound property is guaranteed and therefore the estimates are denoted as strict.

The general procedure to compute the stress $\tilde{\boldsymbol{\sigma}}_{n}$ is summarized in algorithm 1.

\section{Data:}

- Approximate stress field $\boldsymbol{\sigma}_{n}^{H, \Delta t}$,

- K-admissible displacement $\tilde{\mathbf{u}}_{n}$ and

- geometrical information of the finite element mesh (nodes, elements and faces)

Result:

- Equilibrated stress $\tilde{\boldsymbol{\sigma}}_{n}$

// Compute equilibrated interelement tractions

for $i=1, \ldots, N_{\text {no }}$ (loop in nodes $\mathbf{x}_{i}$ ) do

| compute $\mathbf{b}_{i l}, l=1, \ldots, \mathcal{F}\left(\mathbf{x}_{i}\right)$ solving local system (100);

end

(Traction $\mathbf{t}_{n}$ at $\Gamma_{l}, l=1, \ldots, N_{\mathrm{fa}}$ is characterized from the values $\mathbf{b}_{i l}$ )

// Compute equilibrated stress

for $k=1, \ldots, N_{\mathrm{el}}$ (loop in elements $\Omega_{k}$ ) do

| compute the equilibrated stress $\tilde{\boldsymbol{\sigma}}_{n}$ solving the local problems (94); end

Algorithm 1: Computation of equilibrated stresses $\tilde{\boldsymbol{\sigma}}_{n}$ with the hybrid fluxes method. 


\subsubsection{The flux-free method}

The flux free method furnishes equilibrated stresses $\tilde{\boldsymbol{\sigma}}_{n}, n=0, \ldots, N$, fulfilling equations (90) and (91) without requiring any equilibrated tractions to set the boundary conditions of the local problems. That is, the local Neumann problems do not require enforcing any flux on the boundary. This reduces considerably the implementation complexity of the method.

The equilibrated stresses $\tilde{\boldsymbol{\sigma}}_{n}, n=0, \ldots, N$, are generated as a correction of the computed stress $\boldsymbol{\sigma}_{n}^{H, \Delta t}$,

$$
\tilde{\boldsymbol{\sigma}}_{n}^{\mathrm{ff}}:=\mathcal{C}: \boldsymbol{\varepsilon}\left(\tilde{\mathbf{e}}_{n}\right)+\boldsymbol{\sigma}_{n}^{H, \Delta t}
$$

where $\tilde{\mathbf{e}}_{n}$ is an estimate of the error in displacements, computed solving local flux free problems [66]. As for the hybrid fluxes method, the computed stress $\boldsymbol{\sigma}_{n}^{H, \Delta t}$ has to fulfill equation (93) to ensure solvability of the local problems.

For the sake of simplicity, the presentation is restricted to linear elements. In this case, all the nodes $\mathbf{x}_{i}, i=1, \ldots, N_{\mathrm{no}}$, are also mesh vertices. The main rationale of the flux free method is to define function $\tilde{\mathbf{e}}_{n}$ as the addition of local estimates $\tilde{\mathbf{e}}_{n}^{i}$ associated with the mesh vertices, namely

$$
\tilde{\mathbf{e}}_{n}:=\sum_{i=1}^{N_{\mathrm{no}}} \tilde{\mathbf{e}}_{n}^{i} .
$$

Each local estimate $\tilde{\mathbf{e}}_{n}^{i}$ is computed solving a problem defined in the patch $\omega^{i}:=\operatorname{supp}\left(\varphi_{i}\right)$ centered at node $\mathbf{x}_{i}$. The local problem is solved with a refined finite element mesh in the patch $\omega^{i}$. The characteristic element size of this refined mesh is $h<<H$ and the corresponding functional space is denoted by $\mathcal{V}_{\omega^{i}}^{h}$.

The local estimate $\tilde{\mathbf{e}}_{n}^{i}$ is one solution of the problem: find $\tilde{\mathbf{e}}_{n}^{i} \in \mathcal{V}_{\omega^{i}}^{h}$ such that

$$
a\left(\tilde{\mathbf{e}}_{n}^{i}, \mathbf{w}\right)=R_{n}\left(\varphi_{i}\left(\mathbf{w}-\Pi^{H} \mathbf{w}\right)\right) \quad \forall \mathbf{w} \in \mathcal{V}_{\omega^{i}}^{h}
$$

where the weak residual $R_{n}$ stands for

$$
R_{n}(\mathbf{w}):=l_{n}(\mathbf{w})-\left(\rho \ddot{\tilde{\mathbf{u}}}_{n}, \mathbf{w}\right)-\left(\boldsymbol{\sigma}_{n}^{H, \Delta t}, \boldsymbol{\varepsilon}(\mathbf{w})\right) .
$$

Here, the operator $\boldsymbol{\Pi}^{H}: \mathcal{V}_{0} \rightarrow \mathcal{V}_{0}^{H}$ is the interpolation operator in $\mathcal{V}_{0}^{H}$. Once $\tilde{\mathbf{e}}_{n}^{i}$ are computed for $i=1, \ldots, N_{\text {no }}$ solving (103), $\tilde{\mathbf{e}}_{n}$ is recovered using (102) and the stress field $\tilde{\boldsymbol{\sigma}}_{n}$ follows from (101).

It is worth noting that the flux-free method requires that the residual $R_{n}$ fulfills Galerkin orthogonality. It allows introducing the projection $\Pi^{H} \mathbf{w}$ into the residual $R_{n}(\cdot)$ which guarantees the well-possedness (solvability) of the local problems. Note that, if equation (93) holds, then the residual $R_{n}$ introduced in (104) fulfills

$$
R_{n}(\mathbf{w})=0 \quad \forall \mathbf{w} \in \mathcal{V}_{0}^{H}
$$

The flux-free recovered stresses $\tilde{\boldsymbol{\sigma}}_{n}$ are equilibrated in the asymptotic sense, that is fulfilling equilibrium equations (91) but referred to a discrete space associated with the 
reference $h$-mesh. Thus, the estimate provided by $\tilde{\boldsymbol{\sigma}}_{n}$ does not yield a strict upper bound with respect to the exact error, as indicated in theorem 2. Even though, the flux-free estimate furnishes an asymptotic upper bound, that is the bounding properties hold when the element size $h$ of the reference mesh tends to zero. The flux free method leads to strict bounds if the local problems are solved in stresses with a dual formulation, see [70] for details.

The procedure to compute the stress field $\tilde{\boldsymbol{\sigma}}_{n}$ with the flux free approach is detailed in algorithm 2.

\section{Data:}

- Approximate stress field $\boldsymbol{\sigma}_{n}^{H, \Delta t}$

- K-admissible displacement $\tilde{\mathbf{u}}_{n}$ and

- geometrical information of the finite element mesh (nodes and elements).

Result:

- Equilibrated stress $\tilde{\boldsymbol{\sigma}}_{n}$.

// Compute flux-free error estimate

initialize error estimate: $\tilde{\mathbf{e}}_{n}=\mathbf{0}$;

for $i=1, \ldots, N_{\text {no }}$ (loop in nodes $\mathbf{x}_{i}$ ) do

compute the local estimates $\tilde{\mathbf{e}}_{n}^{i}$ solving local systems (103);

add the contribution of $\tilde{\mathbf{e}}_{n}^{i}$ to the global flux free estimate: $\tilde{\mathbf{e}}_{n} \leftarrow \tilde{\mathbf{e}}_{n}+\tilde{\mathbf{e}}_{n}^{i}$;

end

// Compute equilibrated stress

Post-process $\tilde{\mathbf{e}}_{n}$ into $\tilde{\boldsymbol{\sigma}}_{n}=\mathcal{C}: \boldsymbol{\varepsilon}\left(\tilde{\mathbf{e}}_{n}\right)+\boldsymbol{\sigma}_{n}^{H, \Delta t}$

Algorithm 2: Computation of the equilibrated stresses $\tilde{\boldsymbol{\sigma}}_{n}$ with the flux-free method.

\section{Error assessment for timeline-dependent quanti- ties of interest}

\subsection{Timeline-dependent quantities of interest}

Reference [71] introduces a new type of goal-oriented estimates assessing the error in so-called timeline-dependent quantities of interest. These new quantities are scalar time-dependent outputs of the solution instead of single scalar values and are specially well suited to transient problems. Timeline-dependent quantities are associated with a bounded mapping $L_{\mathrm{TL}}^{\mathcal{O}}(\cdot)$ taking a function $\mathbf{w}$ in the solution space $\mathcal{W}$ and returning a time-dependent scalar function, that is

$$
\begin{aligned}
L_{\mathrm{TL}}^{\mathcal{O}}: \mathcal{W} & \longrightarrow \mathcal{L}^{2}(I) \\
\mathbf{w} & \longmapsto L_{\mathrm{TL}}^{\mathcal{O}}(\mathbf{w}) .
\end{aligned}
$$


Note that the functional $L_{\mathrm{TL}}^{\mathcal{O}}(\cdot)$ is a different mathematical object than the functional $L^{\mathcal{O}}(\cdot)$ associated with the standard quantities of interest becasue $L_{\mathrm{TL}}^{\mathcal{O}}(\cdot)$ returns a timedependent scalar function and $L^{\mathcal{O}}(\cdot)$ returns a single scalar value, see figure 9.

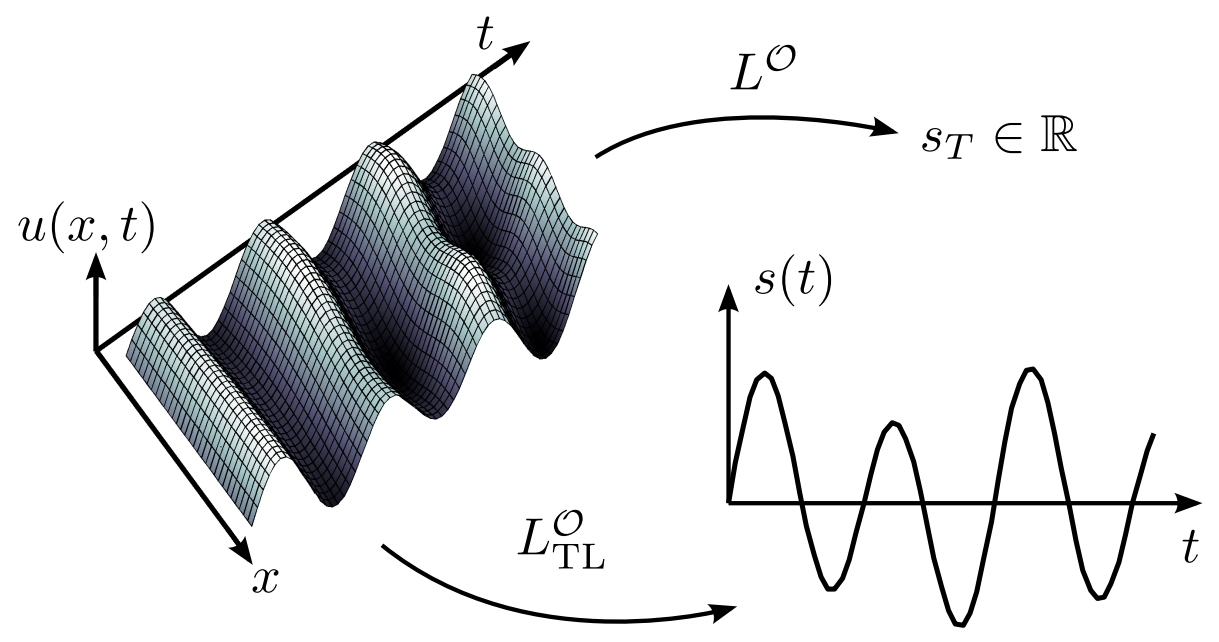

Figure 9: Illustration of scalar and timeline-dependent quantities of interest. The functional $L^{\mathcal{O}}$ maps the time-space solution $\mathbf{u}$ into a scalar value $s_{T} \in \mathbb{R}$. The operator $L_{\mathrm{TL}}^{\mathcal{O}}$ transforms $\mathbf{u}$ into a time-dependent function $s(t)$.

A convenient expression for $L_{\mathrm{TL}}^{\mathcal{O}}(\cdot)$ is defined as an extension of the functional $L^{\mathcal{O}}(\cdot)$ defined in (25),

$\left[L_{\mathrm{TL}}^{\mathcal{O}}(\mathbf{w})\right](t):=\int_{0}^{t}\left(\mathbf{f}^{\mathcal{O}}(\tau), \dot{\mathbf{w}}(\tau)\right) \mathrm{d} \tau+\int_{0}^{t}\left(\mathbf{g}^{\mathcal{O}}(\tau), \dot{\mathbf{w}}(\tau)\right)_{\Gamma_{\mathrm{N}}} \mathrm{d} \tau+\left(\rho \mathbf{v}^{\mathcal{O}}, \dot{\mathbf{w}}(t)\right)+a\left(\mathbf{u}^{\mathcal{O}}, \mathbf{w}(t)\right)$,

where the functions $\mathbf{f}^{\mathcal{O}}$ an $\mathbf{g}^{\mathcal{O}}$ define weighted averages of the solution in the interior domain $\Omega$ or the Neumann boundary $\Gamma_{\mathrm{N}}$, respectively, in the time interval $[0, t]$ for a generic time $t \in I$. On the other hand, functions $\mathbf{v}^{\mathcal{O}}$ and $\mathbf{u}^{\mathcal{O}}$ define weighted averages of the velocities and displacements, respectively, at a generic time point $t \in I$. For the sake of simplicity, the notation $L_{\mathrm{TL}}^{\mathcal{O}}(\mathbf{w} ; t):=\left[L_{\mathrm{TL}}^{\mathcal{O}}(\mathbf{w})\right](t)$ is introduced.

The aim of reference [71] is assessing the quality of the computed timeline-dependent quantity, $\tilde{s}(t):=L_{\mathrm{TL}}^{\mathcal{O}}(\tilde{\mathbf{u}} ; t)$, with respect to the exact quantity of interest, $s(t):=$ $L_{\mathrm{TL}}^{\mathcal{O}}(\mathbf{u} ; t)$. That is, the goal is to assess the error in the quantity of interest which is now a function of time

$$
s^{\mathrm{e}}(t):=s(t)-\tilde{s}(t)
$$

\subsection{Error representation with family of adjoint problems}

Assessing the error in the timeline-quantity $s^{\mathrm{e}}(t)$ requires introducing an error representation similar to the one presented in section 3.4 for the scalar quantity of interest. Thus, an auxiliary problem, analogous to the adjoint problem (24), has to be introduced for the timeline quantity $L_{\mathrm{TL}}^{\mathcal{O}}(\cdot)$. 
This auxiliary problem is defined noting that, for a given time $t \in I$, the value $s(t)=$ $L_{\mathrm{TL}}^{\mathcal{O}}(\mathbf{u} ; t)$ is seen as a scalar quantity of interest taking $t$ as the final time. This scalar quantity of interest is characterized as $L^{\mathcal{O}}(\cdot)=L_{\mathrm{TL}}^{\mathcal{O}}(\cdot ; t)$. Thus, the adjoint problem associated with $L_{\mathrm{TL}}^{\mathcal{O}}(\cdot ; t)$, for a given $t \in I$, is analogous to the one presented in 3.3 and reads: find $\left.\mathbf{u}_{t}^{\mathrm{d}} \in \mathcal{W}\right|_{[0, t]}$ such that

$$
B_{t}\left(\mathbf{w}, \mathbf{u}_{t}^{\mathrm{d}}\right)=\left.L_{\mathrm{TL}}^{\mathcal{O}}(\mathbf{w} ; t) \quad \forall \mathbf{w} \in \mathcal{W}\right|_{[0, t]},
$$

where the bilinear form $B_{t}(\cdot, \cdot)$ is defined as

$$
\begin{aligned}
B_{t}(\mathbf{v}, \mathbf{w}) & :=\int_{0}^{t}\left(\rho\left(\ddot{\mathbf{v}}(\tau)+a_{1} \dot{\mathbf{v}}(\tau)\right), \dot{\mathbf{w}}(\tau)\right) \mathrm{d} \tau+\int_{0}^{t} a\left(\mathbf{v}(\tau)+a_{2} \dot{\mathbf{v}}(\tau), \dot{\mathbf{w}}(\tau)\right) \mathrm{d} \tau \\
& +\left(\rho \dot{\mathbf{v}}\left(0^{+}\right), \dot{\mathbf{w}}\left(0^{+}\right)\right)+a\left(\mathbf{v}\left(0^{+}\right), \mathbf{w}\left(0^{+}\right)\right),
\end{aligned}
$$

and the space $\left.\mathcal{W}\right|_{[0, t]}$ denotes the restriction of $\mathcal{W}$ to the time interval $[0, t]$.

Note that the solution of equation (106) is denoted by $\mathbf{u}_{t}^{\mathrm{d}}$ emphasizing that there is a different solution for each time $t$. Consequently, equation (106) describes a family of problems, one for each time $t$.

Analogously as for the derivation of the adjoint problem for the scalar quantity of interest (26), the associated strong form of problem (106), for the functional $L_{\mathrm{TL}}^{\mathcal{O}}(\cdot)$ defined in (105), is readily derived as

$$
\begin{aligned}
\rho\left(\ddot{\mathbf{u}}_{t}^{\mathrm{d}}-a_{1} \dot{\mathbf{u}}_{t}^{\mathrm{d}}\right)-\boldsymbol{\nabla} \cdot \boldsymbol{\sigma}_{t}^{\mathrm{d}} & =-\mathbf{f}^{\mathcal{O}} \quad \text { in } \Omega \times[0, t], \\
\mathbf{u}_{t}^{\mathrm{d}} & =\mathbf{0} \quad \text { on } \Gamma_{\mathrm{D}} \times[0, t], \\
\boldsymbol{\sigma}_{t}^{\mathrm{d}} \cdot \mathbf{n} & =-\mathbf{g}^{\mathcal{O}} \quad \text { on } \Gamma_{\mathrm{N}} \times[0, t], \\
\mathbf{u}_{t}^{\mathrm{d}} & =\mathbf{u}^{\mathcal{O}} \quad \text { at } \Omega \times\{t\}, \\
\dot{\mathbf{u}}_{t}^{\mathrm{d}} & =\mathbf{v}^{\mathcal{O}} \quad \text { at } \Omega \times\{t\},
\end{aligned}
$$

with the constitutive law

$$
\boldsymbol{\sigma}_{t}^{\mathrm{d}}:=\mathcal{C}: \boldsymbol{\varepsilon}\left(\mathbf{u}_{t}^{\mathrm{d}}-a_{2} \dot{\mathbf{u}}_{t}^{\mathrm{d}}\right)
$$

Recall that the data $\mathbf{f}^{\mathcal{O}}, \mathbf{g}^{\mathcal{O}}, \mathbf{u}^{\mathcal{O}}$ and $\mathbf{v}^{\mathcal{O}}$ enters in the definition of $L_{\mathrm{TL}}^{\mathcal{O}}(\cdot ; t)$ as indicated in (105). Note that for each time $t$, problem (107) is of the same type as (26) and therefore has to be integrated backwards in time. Thus, the family of adjoint problems associated with the timeline-dependent quantity $L_{\mathrm{TL}}^{\mathcal{O}}$ is a family of standard problems in elastodynamics.

For a particular instance of time $t$, the error representation of the timeline-dependent quantity of interest $s^{\mathrm{e}}(t)$ is similar to the standard scalar case but taking the adjoint solution $\mathbf{u}_{t}^{\mathrm{d}}$ related with the particular value $t \in I$, namely

$$
s^{\mathrm{e}}(t)=R_{t}\left(\mathbf{u}_{t}^{\mathrm{d}}\right)
$$

where

$$
\begin{aligned}
R_{t}(\mathbf{w}) & :=L_{t}(\mathbf{w} ; t)-B_{t}(\tilde{\mathbf{u}}, \mathbf{w}) \text { and } \\
L_{t}(\mathbf{w}) & :=\int_{0}^{t} l(\tau ; \dot{\mathbf{w}}(\tau)) \mathrm{d} \tau+\left(\rho \mathbf{v}_{0}, \dot{\mathbf{w}}\left(0^{+}\right)\right)+a\left(\mathbf{u}_{0}, \mathbf{w}\left(0^{+}\right)\right) .
\end{aligned}
$$


Hence, an estimate for $s^{\mathrm{e}}(t)$ is obtained injecting an enhanced adjoint approximation $\tilde{\mathbf{u}}_{t}^{\mathrm{d}}$ in equation (109)

$$
s^{\mathrm{e}}(t) \approx R_{t}\left(\tilde{\mathbf{u}}_{t}^{\mathrm{d}}\right)=: \tilde{s}^{\mathrm{e}} .
$$

Obviously, it is not possible, in practice, to independently compute the infinite solutions $\tilde{\mathbf{u}}_{t}^{\mathrm{d}}$ (one for each time $t \in I$ ) and then using them in equation (109) to assess $s^{\mathrm{e}}(t)$. However, taking $\mathbf{f}^{\mathcal{O}}$ and $\mathbf{g}^{\mathcal{O}}$ constant in time (which accounts for a number of interesting cases), the different functions $\mathbf{u}_{t}^{\mathrm{d}}$ corresponding to different time instances are all equivalent after a time translation. Thus, if $\mathbf{u}_{t}^{\mathrm{d}}$ is properly computed for a particular value of $t$, for instance $t=T$, the general functions $\mathbf{u}_{t}^{\mathrm{d}}$ for $t \neq T$ are easily recovered as a direct post-process of $\mathbf{u}_{T}^{\mathrm{d}}$. This fundamental result, shown in the following theorem, is the crucial observation that allows the error estimation technique to be brought to fruition.

Theorem 5. For a given $t$, let $\mathbf{u}_{t}^{\mathrm{d}}$ be the solution of the adjoint problem defined by equations (107). Assume that data $\mathbf{f}^{\mathcal{O}}$ and $\mathbf{g}^{\mathcal{O}}$ in (105) are constant in time, i.e. $\mathbf{f}^{\mathcal{O}}(\mathbf{x}, t)=\mathbf{f}^{\mathcal{O}}(\mathbf{x})$ and $\mathbf{g}^{\mathcal{O}}(\mathbf{x}, t)=\mathbf{g}^{\mathcal{O}}(\mathbf{x})$.

Then, $\mathbf{u}_{t}^{\mathrm{d}}$ is related with the adjoint solution associated with the final time $T, \mathbf{u}_{T}^{\mathrm{d}}$, via the time translation

$$
\mathbf{u}_{t}^{\mathrm{d}}(\tau)=\mathbf{u}_{T}^{\mathrm{d}}(\tau+T-t) .
$$

A proof of this theorem may be found in [71].

Consequently, The adjoint approximations $\tilde{\mathbf{u}}_{t}^{\mathrm{d}}$ used in the error estimate (110) are computed applying the time shift (111) to the adjoint approximation $\tilde{\mathbf{u}}_{T}^{\mathrm{d}}$ associated with the final time $T$

$$
\tilde{\mathbf{u}}_{t}^{\mathrm{d}}(\tau):=\tilde{\mathbf{u}}_{T}^{\mathrm{d}}(\tau+T-t) .
$$

Thus, only one adjoint approximation $\tilde{\mathbf{u}}_{T}^{\mathrm{d}}$ has to be computed and the others are simply recovered by a time shift.

\subsection{Modal-based adjoint approximation}

The error estimate $\tilde{s}^{\mathrm{e}}(t)$ is computed once the approximation $\tilde{\mathbf{u}}_{T}^{\mathrm{d}} \approx \mathbf{u}_{T}^{\mathrm{d}}$ is available. This section is devoted to the actual computation of $\tilde{\mathbf{u}}_{T}^{\mathrm{d}}$. Note that $\mathbf{u}_{T}^{\mathrm{d}}$ coincides with the adjoint solution $\mathbf{u}^{\mathrm{d}}$ associated with the scalar quantity of interest $L^{\mathcal{O}}(\cdot)$. Consequently, computing $\tilde{\mathbf{u}}_{T}^{\mathrm{d}}$ is equivalent to compute an approximation $\tilde{\mathbf{u}}^{\mathrm{d}} \approx \mathbf{u}^{\mathrm{d}}$.

Function $\tilde{\mathbf{u}}^{\mathrm{d}}$ (or equivalently $\tilde{\mathbf{u}}_{T}^{\mathrm{d}}$ ) is obtained using the standard approximation techniques for elastodynamics. However, if $\tilde{\mathbf{u}}^{\mathrm{d}}$ has to be used for a timeline estimate $\tilde{s}^{\mathrm{e}}(t)$, then, a better option is using modal analysis, see reference [71]. The modal based description of $\tilde{\mathbf{u}}^{\mathrm{d}}$ simplifies the time shift (111) required to assess the error in the timeline quantity and makes the actual computation of $\tilde{s}^{\mathrm{e}}(t)$ more efficient.

Approximating function $\tilde{\mathbf{u}}^{\mathrm{d}}$ with modal analysis requires introducing a semidiscrete version (discrete in space and exact in time) of the adjoint problem (26). The semidiscrete 
problem reads: find $\mathbf{u}^{\mathrm{d}, H, p+1}(t) \in \mathcal{V}_{0}^{H, p+1}$ verifying the final conditions $\mathbf{u}^{\mathrm{d}, H, p+1}(T)=\mathbf{u}^{\mathcal{O}}$ and $\dot{\mathbf{u}}^{\mathrm{d}, H, p+1}(T)=\mathbf{v}^{\mathcal{O}}$ and such that for all $t \in I$

$$
m\left(\ddot{\mathbf{u}}^{\mathrm{d}, H, p+1}(t)-a_{1} \dot{\mathbf{u}}^{\mathrm{d}, H, p+1}(t), \mathbf{w}\right)+a\left(\mathbf{u}^{\mathrm{d}, H, p+1}(t)-a_{2} \dot{\mathbf{u}}^{\mathrm{d}, H, p+1}(t), \mathbf{w}\right)=-l^{\mathcal{O}}(t ; \mathbf{w}),
$$

for all test function $\mathbf{w} \in \mathcal{V}_{0}^{H, p+1}$, where $l^{\mathcal{O}}(t ; \mathbf{w}):=\left(\mathbf{f}^{\mathcal{O}}(t), \mathbf{w}\right)+\left(\mathbf{g}^{\mathcal{O}}(t), \mathbf{w}\right)_{\Gamma_{\mathrm{N}}}$ and $\mathcal{V}_{0}^{H, p+1}$ is the functional space obtained with $p$-refinement of the original functional space $\mathcal{V}_{0}^{H}$.

Remark 6. The spacial resolution of the adjoint approximation $\tilde{\mathbf{u}}^{\mathrm{d}}$ has to be richer than the one of the numerical approximation $\tilde{\mathbf{u}}$. Otherwise, the error is underestimated when plugging the approximation $\tilde{\mathbf{u}}^{\mathrm{d}}$ into the residual $R(\cdot)$ by an effect analogous to Galerkin orthogonality. For that reason the functional space used to define the semidiscrete problem (113) is $\mathcal{V}_{0}^{H, p+1}$ instead of $\mathcal{V}_{0}^{H}$.

A modal-based approximation of the problem (113) is obtained introducing the generalized eigenvalue problem: find $(\tilde{\omega}, \tilde{\mathbf{q}}) \in \mathbb{R} \times \mathcal{V}_{0}^{H, p+1}$ such that

$$
a(\tilde{\mathbf{q}}, \mathbf{w})=(\tilde{\omega})^{2} m(\tilde{\mathbf{q}}, \mathbf{w}) \quad \forall \mathbf{w} \in \mathcal{V}_{0}^{H, p+1}
$$

The $i$-th eigenpair solution of this problem is referred as $\left(\tilde{\omega}_{i}, \tilde{\mathbf{q}}_{i}\right)$. Note that the number of eigenpairs is the number of degrees of freedom in the functional space $\mathcal{V}_{0}^{H, p+1}$, denoted by $N_{\text {dof }}$. Typically, the eigenpairs are sorted from low to high frequencies, namely $\tilde{\omega}_{1} \leq$ $\tilde{\omega}_{2} \cdots \leq \tilde{\omega}_{N_{\mathrm{dof}}}$, and eigenvectors are normalized to be orthonormal with respect the product $m(\cdot, \cdot)$, i.e.

$$
m\left(\tilde{\mathbf{q}}_{i}, \tilde{\mathbf{q}}_{j}\right)=\delta_{i j}, \quad 1 \leq i, j \leq N_{\mathrm{dof}}
$$

The complexity of the system of ODEs resulting from (113) is considerably reduced by expressing the adjoint solution $\mathbf{u}^{\mathrm{d}, H, p+1}(\mathbf{x}, t)$ as a combination of the eigenvectors $\tilde{\mathbf{q}}_{i}, i=1, \ldots, N_{\text {dof }}$, that is

$$
\mathbf{u}^{\mathrm{d}, H, p+1}(\mathbf{x}, t)=\sum_{i=1}^{N_{\text {dof }}} \tilde{\mathbf{q}}_{i}(\mathbf{x}) \tilde{y}_{i}(t) .
$$

Thus, the system of ODEs (113) is transformed into the uncoupled set of scalar ordinary differential equations

$$
\begin{aligned}
\ddot{\tilde{y}}_{i}-\left[a_{1}+a_{2}\left(\tilde{\omega}_{i}\right)^{2}\right] \dot{\tilde{y}}_{i}+\left(\tilde{\omega}_{i}\right)^{2} \tilde{y}_{i} & =\tilde{l}_{i}, \\
\tilde{y}_{i}(T) & =\tilde{u}_{i}, \\
\dot{\tilde{y}}_{i}(T) & =\tilde{v}_{i},
\end{aligned}
$$

where the r.h.s. terms $\tilde{l}_{i}, \tilde{u}_{i}$ and $\tilde{v}_{i}$ are computed using the data characterizing the quantity of interest (105) and the eigenvector $\tilde{\mathbf{q}}_{i}$,

$$
\tilde{l}_{i}(t):=\left(\mathbf{f}^{\mathcal{O}}(t), \tilde{\mathbf{q}}_{i}\right)+\left(\mathbf{g}^{\mathcal{O}}(t), \tilde{\mathbf{q}}_{i}\right)_{\Gamma_{\mathrm{N}}}, u_{i}:=m\left(\mathbf{u}^{\mathcal{O}}, \tilde{\mathbf{q}}_{i}\right) \text { and } v_{i}:=m\left(\mathbf{v}^{\mathcal{O}}, \tilde{\mathbf{q}}_{i}\right) .
$$

The cost of modal analysis scales as, see references $[72,73,74]$,

$$
\mathcal{O}\left(N_{\mathrm{dof}} \cdot N_{\mathrm{bw}}^{2}\right)+\mathcal{O}\left(N_{\mathrm{dof}}^{2} \cdot N_{\mathrm{bw}}\right)+\mathcal{O}\left(N_{\mathrm{dof}}^{3}\right),
$$


where $N_{\mathrm{bw}}$ denotes the half-bandwidth of the finite element matrices associated with the functional space $\mathcal{V}_{0}^{H, p+1}$. Thus, the modal-based approach is not computationally affordable unless the modal description (116) is truncated up to the first $M$ terms, being $M \ll N_{\text {dof }}$. Consequently, the adjoint approximation $\tilde{\mathbf{u}}^{\mathrm{d}}$ is defined as the truncated expansion

$$
\tilde{\mathbf{u}}^{\mathrm{d}}(\mathbf{x}, t):=\sum_{i=1}^{M} \tilde{\mathbf{q}}_{i}(\mathbf{x}) \tilde{y}_{i}(t) .
$$

Note that the number of required vibration modes $M$ has to be selected such that the truncated high frequency modes (for $i>M$ ) are negligible in (116). That is, such that $\tilde{\mathbf{u}}^{\mathrm{d}}$ is a good approximation to $\mathbf{u}^{\mathrm{d}, H}$. This is equivalent to assume that for $i>M$ the values of $\tilde{l}_{i}, \tilde{u}_{i}$ and $\tilde{v}_{i}$, as defined in (118), are close to zero, and consequently $\tilde{y}_{i}(t) \approx 0$. This is guaranteed if the data $\mathbf{f}^{\mathcal{O}}, \mathbf{g}^{\mathcal{O}}, \mathbf{u}^{\mathcal{O}}$ and $\mathbf{v}^{\mathcal{O}}$ are well captured by the expansion of the first $M$ eigenvectors.

Once $\tilde{\mathbf{u}}^{\mathrm{d}}$ (or equivalently $\tilde{\mathbf{u}}_{T}^{\mathrm{d}}$ ) is available, the adjoint family $\tilde{\mathbf{u}}_{t}^{\mathrm{d}}$ is recovered using the time shift (111). Then, $\tilde{\mathbf{u}}_{t}^{\mathrm{d}}$ is plugged in equation (110) furnishing the timeline error estimate $\tilde{s}^{\mathrm{e}}(t)$.

Remark 7. (Illustrative example) This example illustrates the performance of the error estimate $\tilde{s}^{\mathrm{e}}(t)$. The computational domain is the three dimensional structure plotted in figure 10 which is clamped at the supports and it is loaded with the time-dependent traction

$$
\mathbf{g}(t)=\left\{\begin{array}{c}
-g(t) \mathbf{e}_{1} \text { on } \Gamma_{g}, \\
\mathbf{0} \text { elsewhere }
\end{array}\right.
$$

where function $g(t)$ is defined in figure 10 and the values $g_{\max }=1 \cdot 10^{3} \mathrm{~Pa}$ and $t_{g}=$ $1 \cdot 10^{-3} \mathrm{~s}$ are considered. The set $\Gamma_{g}$ is the boundary where the load is applied, see figure 10. The structure is initially at rest $\left(\mathbf{u}_{0}=\mathbf{v}_{0}=\mathbf{0}\right)$ and the body force is zero $(\mathbf{f}=\mathbf{0})$. The material properties are Young's modulus $E=2 \cdot 10^{10} \mathrm{~Pa}$, Poisson's ratio $\nu=0.2$, density $\rho=2.4 \cdot 10^{3} \mathrm{~kg} / \mathrm{m}^{3}$ and viscosity $a_{1}=a_{2}=0$. The final time is $T=0.02 \mathrm{~s}$.
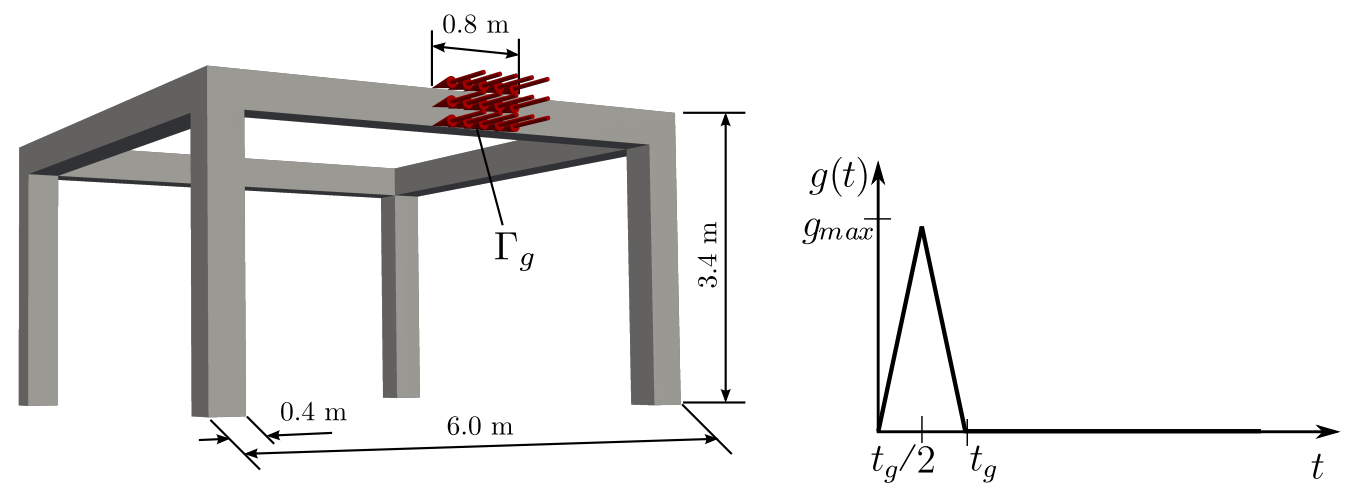

Figure 10: Problem geometry (left) and time description of the external load (right).

This example focuses in the timeline-dependent quantity of interest

$$
s(t):=\frac{1}{\operatorname{meas}\left(\Gamma_{g}\right)}\left(\mathbf{e}_{1}, \mathbf{u}(t)\right)_{\Gamma_{g}},
$$


which is the average of the x-component of the displacement in the boundary $\Gamma_{g}$ at every time $t \in I$.

The problem is discretized with trilinear hexahedra in space and with the Newmark method in time with parameters $\beta=1 / 4$ and $\gamma=1 / 2$. The approximated quantity of interest $\tilde{s}(t)=L^{\mathcal{O}}(\tilde{\mathbf{u}} ; t)$ is computed from the approximate solution $\tilde{\mathbf{u}}$ obtained with the coarse finite element mesh plotted in figure 11 and with $N=400$ time steps. The reference quantity of interest $s(t)=L^{\mathcal{O}}(\mathbf{u} ; t)$ is obtained by assuming that the exact solution $\mathbf{u}$ is fairly replaced by a reference solution obtained using the reference mesh in figure 11 and $N=1600$ time steps. The error in the quantity of interest is evaluated using the reference solution, namely $s^{\mathrm{e}}(t)=s(t)-\tilde{s}(t)$. Finally, the error estimate $\tilde{s}^{\mathrm{e}}(t)$ is computed using up to $M=60$ vibration modes for approximating the adjoints.
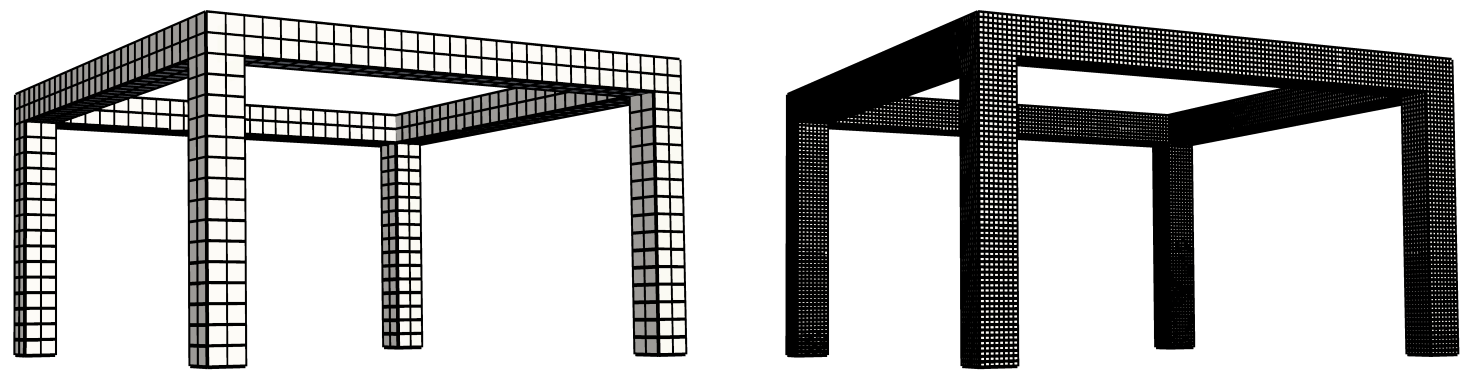

Figure 11: Coarse (left) and reference (right) meshes used in this example with 334 and 22016 elements respectively.

Figure 12 shows the computed and reference timeline-dependent quantities, $\tilde{s}(t)$ and $s(t)$, along with the assessed and reference errors, $\tilde{s}^{\mathrm{e}}(t)$ and $s^{\mathrm{e}}(t)$. Note that the quality of the error estimate $\tilde{s}^{\mathrm{e}}(t)$ increases with the number of vibration modes. For $M=60$ modes, the error estimate $\tilde{s}^{\mathrm{e}}(t)$ and the reference error $s^{\mathrm{e}}(t)$ are in very good agreement.

\section{Closure}

The most significant error assessment techniques for structural transient dynamics are reviewed, namely: recovery-based estimates, dual weighted residuals, constitutive relation error and error assessment for timeline-dependent quantities of interest.

The recovery-based estimates for transient dynamics are an extension of the recovery procedures available for steady state linear elasticity. The classical space recovery allows assessing only the space discretization error. Thus, to carry out adaptive procedures, the time discretization errors have to be accounted independently. Moreover, the standard stress recovery techniques are not sufficient to assess the kinetic energy of the error. Thus, a specific recovery procedure is also introduced for the velocities.

The dual weighted residuals approach produces accurate approximations to the error in the quantity of interest and also provides local error indicators for mesh adaptivity. The error estimate is obtained by plugging an enhanced approximation of the adjoint 

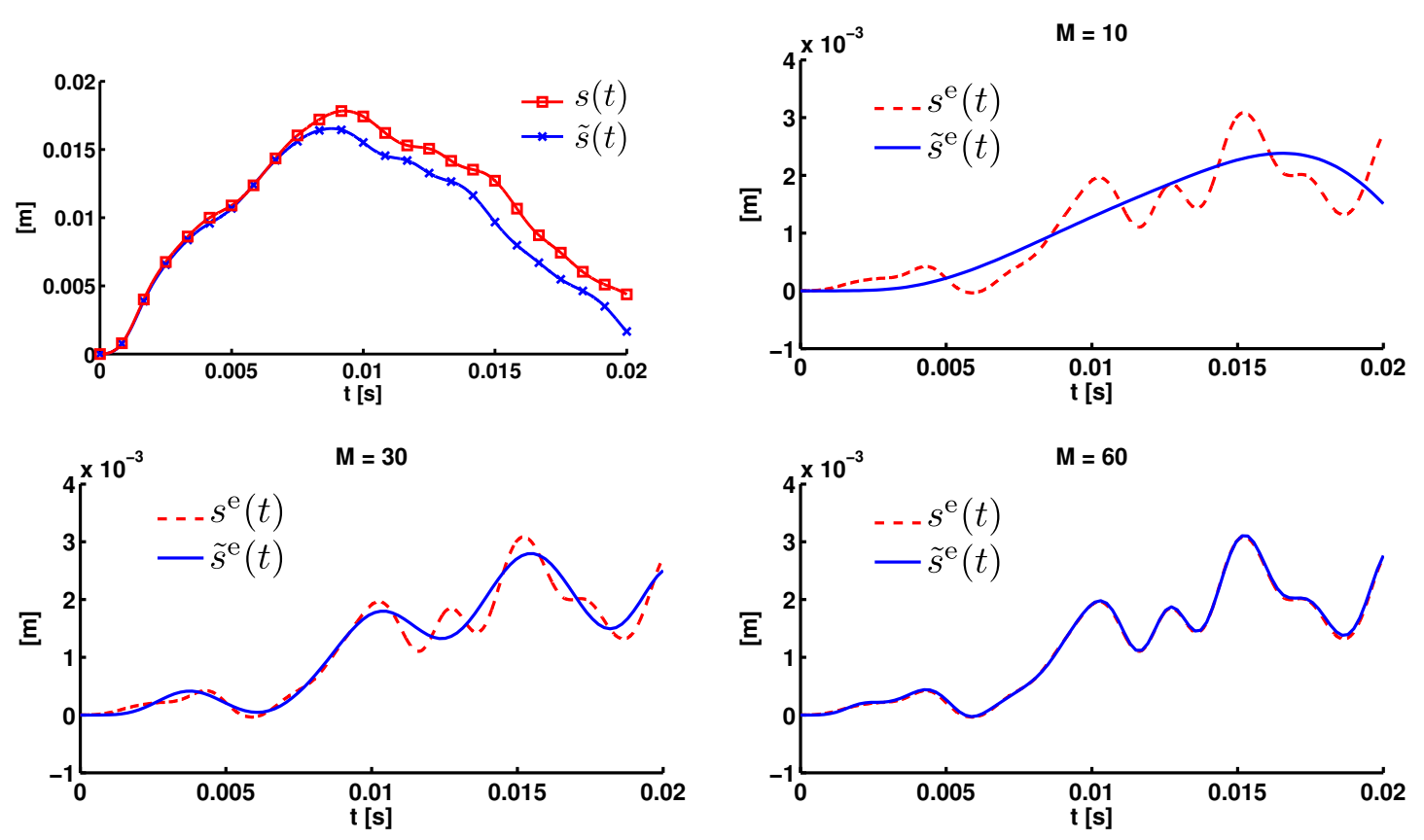

Figure 12: Approximated quantity of interest $\tilde{s}(t)$ and reference quantity $s(t)$ (top, left). Reference and assessed errors, $s^{\mathrm{e}}(t)$ and $\tilde{s}^{\mathrm{e}}(t)$, for three different number of vibration modes for approximating the adjoints, $M=10$ (top, right), $M=30$ (bottom, left) and $M=60$ (bottom, right).

problem into the space-time weak residual associated with the numerical solution. This technique accounts for both the space and time discretization errors and it is used to adapt both space and time grids.

The constitutive relation error estimates furnish bounds of the error both in an energy measure and in the quantity of interest. The extension of this technique to elastodynamics is based in a key hypothesis: the formulation contains a certain amount of damping. Thus, the computed bounds degenerate as the value of the damping tends to zero. Computing the error bounds requires obtaining admissible stress fields for both the original and the adjoint problems.

Finally, an error estimate for the so-called timeline-dependent quantities of interest is described. This kind of quantities are scalar time-dependent functions and are specially well suited to analyze the outcome of transient problems. Although at the first sight this type of quantities require characterizing a family of adjoint problems, approximating the adjoint solution with a modal approach constitutes an efficient and affordable tool to assess them.

\section{Acknowledgment}

Partially supported by Ministerio de Educación y Ciencia, Grant DPI2011-27778-C02-02 and Universitat Politècnica de Catalunya (UPC-BarcelonaTech), grant UPC-FPU. 


\section{References}

[1] I. Babuŝkka and W. C. Rheinboldt. Error estimates for adaptive finite element computations. SIAM J. Numer. Anal., 18:736-754, 1978.

[2] P. Ladevèze and D. Leguillon. Error estimate procedure in the finite element method. SIAM J. on Numerical Analysis, 20:485-509, 1983.

[3] O. C. Zienkiewicz and J. Z. Zhu. A simple error estimator and adaptative procedure for practical engineering analysis. Int. J. Numer. Meth. Engrg., 24:337-357, 1987.

[4] M. Paraschivoiu, J. Peraire, and A. T. Patera. A posteriori finite element bounds for linear-functional outputs of elliptic partial differential equations. Comput. Methods Appl. Mech. Engrg., 150:289-321, 1997.

[5] N. Parés, J. Bonet, A. Huerta, and J. Peraire. The computation of bounds for linearfunctional outputs of weak solutions to the two-dimensional elasicity equations. Comput. Methods Appl. Mech. Engrg., 195:406-429, 2006.

[6] F. Cirak and E. Ramm. A posteriori error estimation and adaptivity for linear elasticity using the reciprocal theorem. Comput. Methods Appl. Mech. Engrg., 156:351362, 1998.

[7] S. Prudhomme and J. T. Oden. On goal-oriented error estimation for elliptic problems: application to the control of pointwise errors. Comput. Methods Appl. Mech. Engrg., 176:313-331, 1999.

[8] M. Ainsworth and J. T. Oden. A posteriori error estimation in Finite element analysis. John Wiley \& Sons Ltd., 2000.

[9] P. Ladevèze and J. P. Pelle. La maîtrise du calcul en mécanique linéaire et non linéaire. Lavoisier, 2001.

[10] E. Stein. Error-controlled adaptive finite elements in solid mechanics. John Wiley \& Sons Ltd., 2003.

[11] M. Ainsworth and J. T. Oden. A posteriori error estimation in finite element analysis. Comput. Methods Appl. Mech. Engrg., 142:1-88, 1997.

[12] T. Gratsch and K. J. Bathe. A posteriori error estimation techniques in practical finite element analysis. Computers and Structures, 83:235-265, 2005.

[13] P. Díez, N. Parés, and A. Huerta. Encyclopedia of Aerospace Engineering, chapter Error estimation and quality control. John Wiley \& Sons Ltd., 2010.

[14] R. Rannacher. Adaptive Galerkin finite element methods for partil differential equations. Journal of Computational and Applied Mathematics, 128:205-233, 2001. 
[15] L. Gallimard, P. Ladeveze, and J. P. Pelle. An enhanced error estimator on the constitutive relation for plasticity problems. Computers and Structures, 78:801$810,2000$.

[16] P. Ladevèze. Constitutive relation errors for fe analysis considering (visco-)plasticity and damage. Int. J. Numer. Meth. Engrg., 52:527-542, 2001.

[17] P. Ladevèze and N. Moës. Adaptive control for finite element analysis in plasticity. Computers and Structures, 73:45-60, 1999.

[18] P. Ladevèze, N. Moës, and B. Douchin. Constitutive relation error estimators for (visco)plastic finite element analysis with softening. Comput. Methods Appl. Mech. Engrg., 176:247-264, 2000.

[19] N. Parés, P. Díez, and A. Huerta. Exact bounds of the advection-diffusion-reaction equation using flux-free error estimates. SIAM J. Sci. Comput., 31:3064-3089, 2009.

[20] F. Larsson, P. Díez, and A. Huerta. A flux-free a posteriori error estimator for the incompressible Stokes problem using a mixed FE formulation. Comput. Methods Appl. Mech. Engrg., 199:2383-2402, 2010.

[21] N. Parés, P. Díez, and A. Huerta. Bounds of functional outputs for parabolic problems. Part I: Exact bounds of the discontinuous galerkin time discretization. Comput. Methods Appl. Mech. Engrg., 197:1641-1660, 2008.

[22] N. Parés, P. Díez, and A. Huerta. Bounds of functional outputs for parabolic problems. Part II: Bounds of the exact solution. Comput. Methods Appl. Mech. Engrg., 197:1661-1679, 2008.

[23] P. Díez and G. Calderón. Goal-oriented error estimation for transient parabolic problems. Comput. Mech., 39:631-646, 2007.

[24] M. G. Larson and F. Bengzon. Adaptive finite element approximation of multiphysics problems. Commun. Numer. Methods Engrg., 24:505-521, 2008.

[25] M. G. Larson, R. Soderlund, and F. Bengzon. Adaptive finite element approximation of coupled flow and transport problems with applications in heat transfer. Int. J. Numer. Meth. Fluids, 57:1397-1420, 2008.

[26] P. W. Fick, E. H. van Brummelen, and K. G. van der Zee. On the adjoint-consistent formulation of interface conditions in goal-oriented error estimation and adaptivity for fluid-structure interaction. Comput. Methods Appl. Mech. Engrg., 199:3369$3385,2010$.

[27] K. G. van der Zee, E. H. van Brummelen, I. Akkerman, and R. de Borst. Goaloriented error estimation and adaptivity for fluid-structure interaction using exact linearized adjoints. Comput. Methods Appl. Mech. Engrg., 200:2738-2757, 2011.

[28] L. Asner, S. Tavener, and D. Kay. Adjoint-based a posteriori error estimation for coupled time-dependent systems. SIAM J. Sci. Comput., 34:2394-2419, 2012. 
[29] X. D. Li and N. E. Wiberg. Implementation and adaptivity of a space-time finite element method for structural dynamics. Comput. Methods Appl. Mech. Engrg., 156:211-229, 1998.

[30] N. E. Wiberg and X. D. Li. Adaptive finite element procedures for linear and non-linear dynamics. Int. J. Numer. Meth. Engrg., 46:178-1802, 1999.

[31] A. Schleupen and E. Ramm. Local and global error estimations in linear structural dynamics. Computers and structures, 76:741-756, 2000.

[32] L. L. Thompson and D. He. Adaptive space-time finite element methods for the wave equation on unbounded domains. Comput. Methods Appl. Mech. Engrg., 194:19472000, 2005.

[33] S. Lahiri, J. Bonet, and J. Peraire. A variationally consistent mesh adaptation method for triangular elements in explicit lagrangian dynamics. Int. J. Numer. Meth. Engng., 82:1073-1113, 2010.

[34] D. Aubry, D. Lucas, and B. Tie. Adaptive strategy for transient/coupled problems. Applications to thermoelasticity and elastodynamics. Comput. Methods Appl. Mech. Engrg., 176:41-50, 1999.

[35] W. Bangerth, M. Geiger, and R. Rannacher. Adaptive Galerkin finite element methods for the wave equation. Computational Methods in Applied Mathematics, 1:3-48, 2010.

[36] W. Bangerth. Adaptive Finite-Elemente-Methoden zur Lösung der Wellengleichung mit Anwendung in der Phisik der Sonne. PhD thesis, Ruprecht-Karls-Universität Heidelberg, 1998.

[37] W. Bangerth and R. Rannacher. Finite element approximation of the acoustic wave equation: error control and mesh adaptation. East-West Journal of Numerical Mathematics, 7:263-282, 1999.

[38] W. Bangerth and R. Rannacher. Adaptive finite element techniques for the acoustic wave equation. Journal of Computational Acoustics, 9:575-591, 2001.

[39] J. P. Combe, P. Ladevèze, and J. P. Pelle. Discretization error estimator for transcient dynamic simulations. Advances in Engrg. Software, 33:553-563, 2002.

[40] J. P. Combe, P. Ladevèze, and J. P. Pelle. Constitutive relation error estimator for transient finite element analysis. Comput. Methods Appl. Mech. Engrg., 176:165$185,1999$.

[41] P. Ladevèze and J. P. Pelle. Estimation of discretization errors in dynamics. Computers and Structures, 81:1133-1148, 2003.

[42] P. Ladevèze and J. Waeytens. Model verification in dynamics trough strict upper bounds. Comput. Methods Appl. Mech. Engrg., 198:1775-1784, 2009. 
[43] J. Waeytens. Contrôle des calculs en dynamique: bornes strictes et pertinents sur une quantié d'intérêt. PhD thesis, LMT-Cachan, 2010.

[44] P. Ladevèze. Strict upper error bounds for computed outputs of interest in computational structural mechanics. Computational Mechanics, 42:271-286, 2008.

[45] J. Waeytens, L. Chamoin, and P. Ladevèze. Guaranteed error bounds on pointwise quantities of interest for transient viscodynamics problems. Computational Mechanics, 49:291-307, 2012.

[46] F. Verdugo and P. Díez. Computable bounds of functional outputs in linear viscoelastodynamics. Comput. Methods Appl. Mech. Engrg., 245-246:313-330, 2012.

[47] D. Fuentes, D. Littlefield, J. T. Oden, and S. Prudhomme. Extensions of goaloriented error estimation methods to simulation of highly-nonlinear response of shock-loaded elastomer-reinforcedstructures. Comput. Methods Appl. Mech. Engrg., 195:4659-4680, 2006.

[48] L. C. Evans. Partial Differential Equations. American Mathematical Society, 1998.

[49] N. M. Newmark. A method of computation for structural dynamics. J. of Engineering Mechanics, 85:67-94, 1959.

[50] K. Eriksson, D. Estep, P. Hansbo, and C. Johnson. Computational Differential Equations. Studentlitteratur, 1996.

[51] T. J. R. Hughes and G. M. Hulbert. Space-time finite element methods for elastodynamics: Formulations and error estimates. Comput. Methods Appl. Mech. Engrg., 66:339-363, 1988.

[52] G. M. Hulbert and T. J. R. Hughes. Space-time finite element methods for secondorder hypeerbolic equations. Comput. Methods Appl. Mech. Engrg., 84:327-348, 1990.

[53] C. Johnson. Discontinuous galerkin finite element methods for second order hyperbolic problems. Comput. Methods Appl. Mech. Engrg., 107:117-129, 1993.

[54] C. Johnson. Numerical solution of partial differential equations by the finite element method. Cambridge University Press, 1990.

[55] P. Díez and G. Calderón. Remeshing criteria and proper error representations for goal oriented h-adaptivity. Comput. Methods Appl. Mech. Engrg., 196:719-733, 2007.

[56] O. C. Zienkiewicz and Y. M. Xie. A simple error estimator and adaptive time stepping procedure for dynamic analysis. Earthquake Engineering 85 Structural Dynamics, 20:871-887, 1991.

[57] X. D. Li, L. F. Zeng, and N. E. Wiberg. A simple error estimator and adaptive time stepping procedure for dynamic analysis. Com. Num. Meth. Engng., 9:273-292, 1993. 
[58] O. C. Zienkiewicz and J. Z. Zhu. The superconvergent patch recovery and a posteriori error estimates. part 1: The recovery technique. Int. J. Numer. Meth. Engrg., 33:1331-1364, 1992.

[59] O. C. Zienkiewicz and J. Z. Zhu. The superconvergent patch recovery and a posteriori error estimates. Part 2: Error estimates and adaptivity. Int. J. Numer. Meth. Engrg., 33:1365-1382, 1992.

[60] N. E. Wiberg and X. D. Li. Superconvergent patch recovery of finite-element solution and a posteriori $\mathrm{L}_{2}$ norm error estimate. Com. Num. Meth. Engng., 10:313-320, 1994.

[61] X. D. Li and N. E. Wiberg. A posteriori error estimate by element patch postprocessing, adaptive analysis in energy and $\mathrm{L}_{2}$ norms. Computers and Structures, 53:907-919, 1994.

[62] N. E. Wiberg, R. Bausys, and P. Hager. Adaptive h-version eigenfrequency analysis. Computers and structures, 71:565-584, 1999.

[63] R. Rannacher and F. T. Stuttmeier. A feed-back approach to error control in finite element methods: application to linear elasticity. Comput. Mech., 19:434-446, 1997.

[64] W. Bangerth and R. Rannacher. Adaptive Finite Element Methods for Differential Equations. Birkhäuser, 2003.

[65] J. T. Oden and S. Prudhomme. Goal-oriented error estimation and adaptivity for the finite element method. Computers and Math. with Appl., 41:735-765, 2001.

[66] N. Parés, P. Díez, and A. Huerta. A subdomain-based flux-free a posteriori error estimators. Comput. Methods Appl. Mech. Engrg., 195:297-323, 2006.

[67] J. P. Moitinho de Almeida and O. J. B. Almeida Pereira. Upper bounds of the error in local quantities using equilibrated and compatible finite element solutions for linear elastic problems. Comput. Methods Appl. Mech. Engrg., 195:279-296, 2006.

[68] O. J. B. Almeida Pereira and J. P. Moitinho de Almeida. Dual adaptive finite element refinement for multiple local quantities in linear elastostatics. Int. J. Numer. Meth. Engrg., 83:347-365, 2010.

[69] F. Pled, L. Chamoin, and P. Ladevèze. On the techniques for constructing admissible stress fields in model verification: Performances on engineering examples. Int. J. Numer. Meth. Engng., 88:409-441, 2011.

[70] R. Cottereau, P. Díez, and A. Huerta. Strict error bounds for linear solid mechanics problems using a subdomain-based flux-free method. Comput. Mech., 44:533-547, 2009.

[71] F. Verdugo, N. Parés, and P. Díez. Modal based goal-oriented error assessment for timeline-dependent quantities in transient dynamics. Int. J. Numer. Meth. Engng. In print. 
[72] A. Rafique, N. Kapre, and G. A. Constantinides. A high throughput FPGA-based implementation of the lanczos method for the symmetric extremal eigenvalue problem. In 8th International Symposium on Applied Reconfigurable Computing, January 2012.

[73] K. J. Bathe. Finite Element Procedures. Prentice Hall, 1996.

[74] L. N. Trefethen and D. Bau. Numerical linear algebra. Society for Industrial \& Applied Mathematics, 1997. 